\title{
Global ozone monitoring by occultation of stars: an overview of GOMOS measurements on ENVISAT
}

\author{
J. L. Bertaux ${ }^{1}$, E. Kyrölä ${ }^{2}$, D. Fussen ${ }^{3}$, A. Hauchecorne ${ }^{1}$, F. Dalaudier ${ }^{1}$, V. Sofieva ${ }^{2}$, J. Tamminen ${ }^{2}$, F. Vanhellemont ${ }^{3}$, \\ O. Fanton d'Andon ${ }^{4}$, G. Barrot ${ }^{4}$, A. Mangin ${ }^{4}$, L. Blanot ${ }^{4}$, J. C. Lebrun ${ }^{1}$, K. Pérot ${ }^{1}$, T. Fehr ${ }^{5}$, L. Saavedra ${ }^{6}$, \\ G. W. Leppelmeier ${ }^{2}$, and R. Fraisse ${ }^{7}$ \\ ${ }^{1}$ LATMOS-IPSL, CNRS/INSU, UMR 8190, Univ. Versailles St-Quentin, Guyancourt, 78280, France \\ ${ }^{2}$ Finnish Meteorological Institute, Earth Observation, Helsinki, Finland \\ ${ }^{3}$ Institut d'Aeronomie Spatiale de Belgique, Brussels, Belgium \\ ${ }^{4}$ ACRI-st, Sophia-Antipolis, France \\ ${ }^{5}$ ESA, Esrin, Frascati, Italy \\ ${ }^{6}$ IDEAS, Serco, Frascati, Italy \\ ${ }^{7}$ EADS-Astrium, Toulouse, France
}

Received: 30 January 2010 - Published in Atmos. Chem. Phys. Discuss.: 16 April 2010

Revised: 25 October 2010 - Accepted: 3 November 2010 - Published: 20 December 2010

\begin{abstract}
GOMOS on ENVISAT (launched in February, 2002) is the first space instrument dedicated to the study of the atmosphere of the Earth by the technique of stellar occultations (Global Ozone Monitoring by Occultation of Stars). Its polar orbit makes good latitude coverage possible. Because it is self-calibrating, it is particularly well adapted to long time trend monitoring of stratospheric species. With 4 spectrometers, the wavelength coverage of $248 \mathrm{~nm}$ to $942 \mathrm{~nm}$ enables monitoring ozone, $\mathrm{H}_{2} \mathrm{O}, \mathrm{NO}_{2}, \mathrm{NO}_{3}$, air density, aerosol extinction, and $\mathrm{O}_{2}$. Two additional fast photometers (with $1 \mathrm{kHz}$ sampling rate) enable the correction of the effects of scintillations, as well as the study of the structure of air density irregularities resulting from gravity waves and turbulence. A high vertical resolution profile of the temperature may also be obtained from the time delay between the red and the blue photometer. Noctilucent clouds (Polar Mesospheric Clouds, PMC) are routinely observed in both polar summers and global observations of $\mathrm{OClO}$ and sodium are achieved.

The instrument configuration, dictated by the scientific objectives' rationale and technical constraints, is described, together with the typical operations along one orbit, along with the statistics from over 6 years of operation. Typical atmospheric transmission spectra are presented and some retrieval difficulties are discussed, in particular for $\mathrm{O}_{2}$ and $\mathrm{H}_{2} \mathrm{O}$.
\end{abstract}

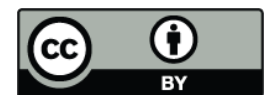

Correspondence to: J. L. Bertaux (bertaux@latmos.ipsl.fr)
An overview is presented of a number of scientific results already published or found in more detail as companion papers in the same ACP GOMOS special issue. This paper is particularly intended to provide an incentive for the exploitation of GOMOS data available to the whole scientific community in the ESA data archive, and to help GOMOS data users to better understand the instrument, its capabilities and the quality of its measurements, thus leading to an increase in the scientific return.

\section{Introduction}

GOMOS (Global Ozone Monitoring by Occultation of Stars) is the first space instrument specifically designed and dedicated to the study of the atmosphere of the Earth by the technique of stellar occultation. It is placed on board the European Space Agency's (ESA) 8.2 ton ENVISAT space platform (ENVIronmental SATellite) among eight other Earth Observation instruments. Together with two other instruments (MIPAS, Michelson Interferometer for Passive Atmospheric Sounding, Fischer et al., 2008, and SCIAMACHY, Scanning Imaging Absorption Spectrometer for Atmospheric Chartography, Bovensmann et al., 1999), GOMOS forms a trio of European instruments fully dedicated to the study of atmospheric chemistry. One role of this trio is to collect the corpus of ozone data (and other atmospheric parameters) necessary to answer the following questions, among others:

Published by Copernicus Publications on behalf of the European Geosciences Union. 
- What is the time evolution of ozone in the stratosphere (i.e. the trend) all over the world?

- What is the effect of release limitations on CFCs?

- Is the measured ozone trend equal to the trend predicted by our best simulation model predictions?

- If not, is there anything in the data that can indicate where are the shortcomings of our models and how can they be improved for more reliable predictions?

Among the three instruments devoted to atmospheric chemistry, the particular role of GOMOS is to establish the 3-D distribution of ozone in the stratosphere and to monitor its trend as a function of altitude, thanks to the particularly high absolute accuracy offered by the technique of occultation of stars, which is also rather immune to the evolution of calibration parameters.

ENVISAT was launched from French Guiana (Kourou) on 28 February 2002, and placed in a circular orbit at $800 \mathrm{~km}$ altitude, almost polar ( $98.55^{\circ}$ inclination), with a period of $100 \mathrm{mn}$. The orbit is helio-synchronous with a descending node (equator crossing) at 10:00 a.m. Local Time. The first stellar occultation with GOMOS was performed in March 2002, and since then GOMOS has worked almost continuously (with a few gaps that will be discussed later). By June 2009, more than 600000 occultations had been collected, obtaining the same number of vertical profiles all over the globe of ozone, $\mathrm{NO}_{2}, \mathrm{NO}_{3}, \mathrm{H}_{2} \mathrm{O}$, aerosol-extinction, $\mathrm{O}_{2}$, temperature and turbulence parameters.

GOMOS was first proposed by a group of European scientists (Bertaux et al., 1988), in response to the Announcement of Opportunity for the Polar Platform issued by ESA for earth observation instrumentation. After peer review, GOMOS was selected together with MIPAS and SCIAMACHY, marking the European will to initiate a major effort in atmospheric research in the aftermath of the Antarctic ozone hole discovery. An industrial Phase A study was conducted by Matra Marconi Space for CNES (Centre National d'Etudes Spatiales) in 1989-1990. Then it was decided at the ESA countries' ministerial conference of Madrid in 1992 that GOMOS would fly on the ENVISAT platform as an ESA-funded instrument. The GOMOS instrument was developed for ESA by an industrial consortium led by Astrium Toulouse (now a part of EADS Astrium). ESA formed a Scientific Advisory Group (SAG) composed of scientists from various European countries to provide advice on the performance requirements of the instrument and to develop the principles of the data inversion and retrieval algorithms.

GOMOS is the first space instrument specifically designed and built to perform atmospheric measurements by stellar occultations: the UVISI instrument (Ultraviolet and Visible Imagers and Spectrometers) on the Midcourse Space Experiment (MSX), which already provided very interesting

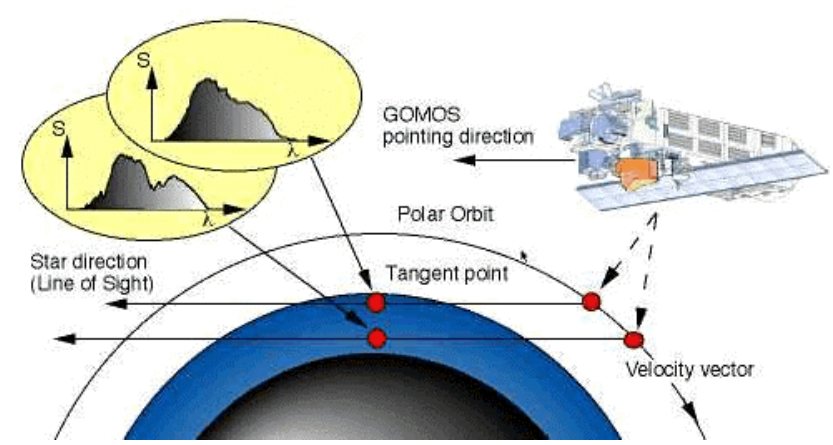

Fig. 1. The GOMOS principle of measurement by occultation of a star. The spectrum of a star is first measured outside of the atmosphere, then through the atmosphere; the ratio of the two spectra is the atmospheric transmission in this spherical geometry, at altitude $z$.

results with this technique (Yee et al., 2002), was not designed for this purpose, and required that the full $6 \mathrm{t}$ spacecraft be oriented to the star, while GOMOS has a moving mirror that is oriented successively toward the various stars to be occulted without perturbing the other Earth Observation instruments. The general principle of the stellar occultation method is illustrated in Fig. 1 and is described below with the particular case of GOMOS.

ENVISAT is 3-axis stabilized, nadir pointing, with a yaw steering law to compensate for Earth rotation and push-broom instruments. GOMOS is implemented on the nadir face of ENVISAT, looking backwards (opposite to the velocity vector), which makes it possible to track near the horizon the successive setting down of various stars while the ENVISAT platform is moving along its orbit. When the star is high above the horizon (typically $z>110 \mathrm{~km}$ ), the light spectrum of the star So $(\lambda)$ is recorded by GOMOS, free of any atmospheric absorption. A few seconds later, the light spectrum of the same star seen through the atmosphere (just above the horizon) is again recorded. The spectrum $S(\lambda, z)$ is modified by the absorption of all atmospheric constituents integrated over the line of sight from ENVISAT to the star according to the Beer-Lambert law,

$S(\lambda, z)=\operatorname{So}(\lambda) \exp \left(-\sigma_{\lambda} N(z)\right)$

where $\lambda$ is the wavelength, $z$ is the minimum altitude of the line of sight above the horizon (tangent height, or tangent altitude), $N(z)\left(\mathrm{mol} \mathrm{cm}^{-2}\right)$ is the integral of ozone density along the line of sight (slant density) and $\sigma_{\lambda}$ the absorption cross section of ozone. Here, only ozone absorption is considered for clarity.

From Eq. (1) one derives:

$N(z)=-\frac{1}{\sigma_{\lambda}} \log \frac{S(\lambda, z)}{S_{0}(\lambda)}$

During one single occultation, a series of tangential column (slant, or line) densities $N\left(z_{j}\right)$ are obtained at various 
tangent altitudes, $z_{j}$. This series can be inverted (vertical inversion) to yield the vertical distribution of the local density $n(z)$ of $\mathrm{O}_{3}\left(\mathrm{~mol} \mathrm{~cm}^{-3}\right)$, assuming that the atmosphere is locally spherically symmetric. This vertical inversion is (in principle) straightforward with the so-called onion-peeling technique. Some care must be taken during the vertical inversion, in order to avoid strong spurious oscillations in the vertical profile $n(z)$, as described in Kyrölä et al. (2010a).

Besides the extreme simplicity of the retrieval algorithm when compared to other methods, the occultation technique has one enormous advantage readily seen from the mathematical form of Eq. (1): an absolute estimate of $n(z)$ is obtained from the ratio of two measurements taken with the same instrument at a few seconds of interval. The method is inherently self-calibrating, and even if the spectral sensitivity of the instrument is changing with time, the ratio will be measured correctly and hence the slant density $N(z)$ (sometimes called also line densities, in short for "density integrated along the Line of Sight, LOS", or slant densities). This protection against long-term drift is of course ideal for the study of trends of ozone and other constituents. The primary measurement of GOMOS is this ratio, which is the spectral transmission of the atmosphere as a function of the tangent height of the line of sight. It is the main data in the Level 1 data product of GOMOS.

In principle, one could use a detector for only one single wavelength $\lambda$, if ozone were the only absorbing species at this wavelength. This is not the case in the UV-VisibleNIR region that can be accessed with CCD silicon detectors, as shown on Fig. 2, which displays the cross-section of a number of atmospheric constituents. Therefore, in order to properly identify each of the atmospheric absorbers separately, one has to measure in a wide wavelength domain with a sufficient spectral resolution. Equation (1) is modified as follows:

$S(\lambda, z)=\operatorname{So}(\lambda) \exp \left(-\sum \sigma_{i}(\lambda) N_{i}(z)\right)$

where $\sigma_{i}(\lambda)$ and $N_{i}$ are the cross-section and the line density of each constituent, $i$, respectively. Therefore, the first step of the Level 2 processing before the vertical inversion will be the retrieval of each quantity $N_{i}(z)$ for each measured altitude. One needs to measure at least as many different wavelengths as the number of constituents. Since the quantum efficiency of Silicon matches nicely the wavelength domain where ozone absorbs (a consideration which dictated the choice of these detectors for GOMOS), we decided to cover a large range of the UV-visible part of the spectrum with a continuous coverage and many spectral pixels, and a least-square model fit to the measured transmission at each altitude allows simultaneous retrieval of all the $N_{i}(z)$ for this altitude; this is the so-called spectral inversion, or separation of constituents (Kyrölä et al., 2010a). Then the vertical inversion is performed separately for each constituent. Within the spectral range of GOMOS (Fig. 4,

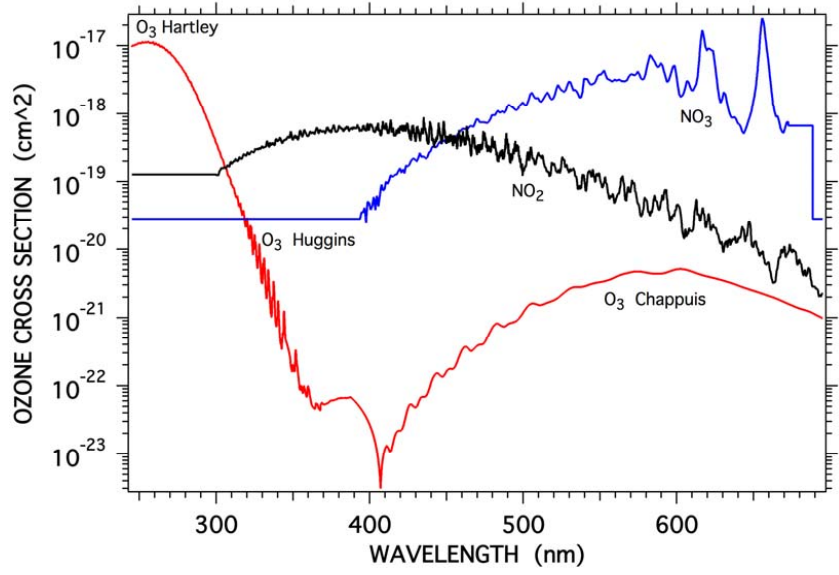

Fig. 2. Cross-sections of ozone (red), $\mathrm{NO}_{2}$ (black) and $\mathrm{NO}_{3}$ (blue) at a temperature of $250 \mathrm{~K}$ used in the GOMOS spectral inversion. Cross-sections at other temperatures are also used. The Huggins bands are particularly sensitive to temperature.

Table 1), $\mathrm{O}_{3}, \mathrm{NO}_{2}, \mathrm{NO}_{3}, \mathrm{OClO}$, air, $\mathrm{O}_{2}, \mathrm{H}_{2} \mathrm{O}$, and aerosols may be measured as a function of altitude. Except for aerosols (and Rayleigh extinction by air), each of these chemical species absorbers has a well defined absorption spectral signature, which facilitates disentangling it from the combined spectrum of the atmospheric transmission (Figs. 2 and 12).

Another important feature of stellar occultations is that the altitude of the measurement is entirely defined by the direction of the star and by the position of the spacecraft. ENVISAT position is known with an accuracy better than $\pm 30 \mathrm{~m}$, ensuring the same accuracy on the knowledge of $z$. The altitude accuracy is important, because an error of $100 \mathrm{~m}$ would result in a $2 \%$ error in ozone density estimate in the altitude region above the peak of ozone, where there is a strong vertical gradient. At variance with other limb emission techniques, the altitude of the measurement does not depend on the attitude of the spacecraft and instrument, therefore avoiding effects from long term drift of the accurate attitude knowledge ( 1 arcsec is projected at the distance of the limb, $\sim 3400 \mathrm{~km}$, as $\Delta z=20 \mathrm{~m})$. The vertical resolution is determined by the time of integration and the vertical velocity of the tangent point, which for GOMOS are $0.5 \mathrm{~s}$ and $3.4 \mathrm{~km} \mathrm{~s}^{-1}$ (maximum), respectively, resulting in a typical vertical sampling resolution of $1.7 \mathrm{~km}$ at most, and less for stars occulted away from the orbital plane. In addition, at lower altitudes where refraction is significant, the vertical velocity is smaller (sampling is higher), but the light path to the star is no longer a straight line, and the exact altitude probed by the ray path depends on the atmospheric air density profile.

The solar occultation technique shares in common with stellar occultations the fact that an absolute quantity is derived from a relative measurement. The Sage-II and 
POAM instruments have been very successful for research of the Earth's atmosphere, and much of what we know at present about the ozone trend as a function of altitude comes from the comparison of Sage-II results on a long time scale. The many wavelengths that are used by GOMOS (instead of the 7 or 9 discrete channels for Sage-II and POAM) allows a much safer identification of all absorbers. We note that the more recent version SAGE-III, flying since February 2002 (Thomason and Taha, 2003) contains many more spectral channels than its predecessors SAM II, SAGE and SAGE-II, all dedicated to solar occultation probing of the atmosphere. The use of stars by GOMOS instead of the sun has some additional favourable features:

- The altitude knowledge of the measurement is almost perfect, while for the solar disk extended source, the pointing of the instrument must be known accurately, and preferably the same portion of the sun must always be observed during the occultation (as the disk brightness is not uniform, especially in the UV).

- The solar occultation occurs at dawn or dusk local time, when the photo-chemistry regime of the atmosphere is changing rapidly (even along the LOS), while the LOS to a star pertains generally to a well defined regime (day or night).

- From the sun-synchronous orbit of ENVISAT, only a limited range of latitudes (around high latitudes) are accessible for solar occultations, while stars allow observation at all latitudes.

There are some drawbacks with the use of stars: they are weak sources, requiring more photon collecting areas. They have different brightness and spectra, which makes the error bars dependent on the magnitude of the star. They are not evenly distributed in the sky, and the sampling of the atmosphere is not as regular as the sampling of limb-looking or nadir-looking instruments (like MIPAS and SCIAMACHY, for example). Night-side measurements are preferred, because on the day-side, the bright limb intensity is competing with the stellar flux. Nevertheless, the inherent advantages of the stellar occultation method overcome these drawbacks by a large margin for the study of trends.

During the commissioning phase of the instrument and the development of data processing software, two unanticipated problems were encountered that complicated somewhat the data retrieval scheme and the operations of GOMOS. One is related to the CCDs' Dark Current (DC) behaviour, which increased significantly under the influence of energetic protons and moreover showed a variable behaviour (known as Random Telegraph Signal, RTS). This phenomenon annihilated completely the advantages of the so-called MPP technology fostered by the CCD provider, EEV Ltd, which in principle should have made possible the use of CCDs at room temperature. Counter measures were taken by setting the
CCDs' at the lowest possible temperature of $+3{ }^{\circ} \mathrm{C}$ allowed by the GOMOS thermal control system, and by collecting at each orbit a measurement of the DC of all pixels, with a deep space observation (no star, in the middle of the night, near equator crossing).

The other problem is related to scintillation of the star, due to small-scale vertical temperature fluctuations of the atmosphere, and associated turbulence. The proposing team (Bertaux et al., 1988) anticipated that the shape of the spectrum of the star would be somewhat affected by scintillations due to the chromatic character of refraction, but thought that this effect could be corrected with the signal of two fast photometers $(1 \mathrm{kHz}$ sampling) recorded simultaneously. It was discovered from GOMOS data that this is perfectly true when the star setting is observed near the orbital plane (because the line of sight stays in the same vertical plane, the orbital plane, and probes the same atmosphere at all wavelengths), but does not hold any longer with an oblique occultation, revealing the existence of isotropic turbulence (Dalaudier et al., 2001; Sofieva et al., 2009b). This new effect decreases somewhat the ozone retrieval accuracy mainly in the altitude region 20 to $40 \mathrm{~km}$ for the brightest stars, and forced us to modify the baseline spectral inversion scheme (which was defined prelaunch), in the direction of a modified DOAS (Differential Optical Absorption Spectroscopy) for $\mathrm{NO}_{2}$ and $\mathrm{NO}_{3}$ (See Sect. 5.5.4).

In spite of these unexpected difficulties, the excellent quality of the GOMOS results makes us still believe that the stellar occultation technique is the best method to achieve the high accuracy measurements necessary to detect small time variations of ozone. It should be mentioned that not only ozone local densities may be monitored for trends, but also the slant densities $N(z)$ of ozone (and other constituents) are archived and available for trend studies. Also, the transmission of the atmosphere can be monitored as such; and after all, it is the UV transmission of the stratosphere that has the greatest impact on living matter on Earth.

The GOMOS data are made available by ESA to the whole scientific community. The goal of this paper is to provide a rather detailed and comprehensive overview of the GOMOS capabilities and the scientific questions that may be addressed, based on the presentation and discussion of some typical results obtained after 7 years of operation, and some improvements in the retrieval algorithms. Another goal is to encourage the community to extract interesting results from the GOMOS data, which are freely available through the ESA distribution system.

In Appendix A a brief history is presented of the stellar occultation technique, with a possible explanation for some strange results that were found for ozone during early attempts with the astronomical satellites OAO-2 and Copernicus in the middle 70s.

An early estimate of GOMOS ozone trend performance may be found in Bertaux et al. (1991), while the previous 
most comprehensive description of the GOMOS instrument and its science objectives may be found in the report of the ESA-formed Scientific Advisory Group (SAG) for GOMOS, written before launch, and summarizing about 10 years of activity of this group for the preparation of the GOMOS investigation (Bertaux et al., 2001, SAG Report ESA SP-1244.). The present paper includes actual in-flight performances, observations and a brief overview of scientific results.

This paper is one of a series of 12 papers proposed to Atmospheric Chemistry and Physics for a GOMOS Special Issue of ACP. Other papers of this special issue cover various subjects: the retrieval algorithms (Kyrölä et al., 2010a), the data characterization and error estimation (Tamminen et al., 2010), the influence of scintillations on GOMOS ozone retrieval (Sofieva et al., 2009), ozone, $\mathrm{NO}_{2}$ and $\mathrm{NO}_{3}$ measurements in the stratosphere and mesosphere (Kyrölä et al., 2010b), the influence of $\mathrm{QBO}$ on tropical ozone, $\mathrm{NO}_{2}$ and $\mathrm{NO}_{3}$ as a function of altitude (Hauchecorne et al., 2010), a climatology of the Tertiary Ozone Maximum (Sofieva et al., 2009), Polar Mesospheric Clouds (PMCs, Pérot et al., 2010), aerosols (Vanhellemont et al., 2010), simultaneous measurements of $\mathrm{OClO}, \mathrm{NO}_{2}$ and $\mathrm{O}_{3}$ in the Arctic polar vortex (Tétard et al., 2010), a climatology of mesospheric sodium (Fussen et al., 2010), and a comparison of GOMOS ozone retrieval with some ground-based measurements (Keckhut et al., 2010).

The rest of the paper is organized as follows: The next section describes how the GOMOS science objectives were turned into Instrument Requirements. Section 3 contains a description of the instrument, of the operations and coverage, together with a sample of typical observations results: star spectra, transmission spectra and scintillations. Section 4 describes some problems encountered during the GOMOS mission, while the retrieval algorithms and error estimates are described in Sect. 5. Section 6 presents some validation results of GOMOS products. Section 7 contains an overview of GOMOS results obtained up to now (ozone, $\mathrm{NO}_{2}, \mathrm{NO}_{3}$, $\mathrm{OClO}$, aerosols, gravity waves and isotropic turbulence, sodium, $\mathrm{PMCs}$ ), and difficulties associated with $\mathrm{O}_{2}$ and $\mathrm{H}_{2} \mathrm{O}$ observations. A short conclusion, calling for a continuation of the GOMOS mission on smaller free flyers, is followed by an Appendix containing a short history of the stellar occultation technique for probing planetary atmospheres.

\section{GOMOS science objectives and instrument requirements}

\subsection{Overview}

In this section we describe the rationale for the instrument configuration of GOMOS, which is a mixture of scientific reasons and considerations of technological constraints. The main objective of long term monitoring of ozone calls for the most accurate measurements of ozone and is the driving factor in the whole specification of GOMOS. The very large variation of the ozone cross section over the UV-visible domain (Fig. 2) offers then a huge dynamic range for the measurement of the slant density of ozone, extending from the tropopause up to the lower thermosphere at $z>100 \mathrm{~km}$. The large cross-section in the UV, and its moderate cross-section in the visible (Chappuis bands, quite extended in wavelength) matches very well the sensitivity of silicon CCDs. This defined the spectral range of the main spectrometer A of GOMOS: the shortest wavelength at $248 \mathrm{~nm}$ is below the maximum cross section of ozone in the UV $(255 \mathrm{~nm})$, and the longest wavelength goes as far as possible in the visible Chappuis band, still compatible with the design of a single spherical holographic grating. The longest wavelength is therefore at $690 \mathrm{~nm}$. However, since the CCD sensitivity in the UV needs a special treatment which does not favour the sensitivity in the visible, the whole UV-visible domain was split in two parts, impinging on two different CCDs, one having the UV enhancement treatment and covering the domain 248 to $389 \mathrm{~nm}$ (CCD A1), while the visible CCD covers the range from $389 \mathrm{~nm}$ to $690 \mathrm{~nm}$ (CCD A2).

The desired spectral resolution was dictated by two different considerations. On one hand, the Huggins bands at $315-350 \mathrm{~nm}$ are highly structured and the cross section there depends strongly on the temperature (Fig. 3). If the spectral resolution would not permit resolving these bands, then a minute (and undetected) long term temperature trend would affect the cross-section there, inducing a spurious ozone trend. On the other hand, it was desirable to have a relatively good resolution in order to detect the trend of any other absorber, known or unknown, with a distinct spectral signature somewhere in the ozone band that could also affect the trend of retrieved ozone. All in all, the sampling of spectrometer A is $0.312 \mathrm{~nm}$ per pixel, and the resolution is $0.8 \mathrm{~nm}$ at FWHM.

Within the sensitivity range of silicon CCDs, it was noticed that $\mathrm{O}_{2}$ and $\mathrm{H}_{2} \mathrm{O}$ have strong absorption bands and their relevance to the ozone problem justified the inclusion of Spectrometer B, also divided in CCD B1 for $\mathrm{O}_{2}$ at $760 \mathrm{~nm}$ and CCD B2 at $936 \mathrm{~nm}$ (see later in this section), with a sampling of $0.047(0.056) \mathrm{nm}$ and a resolution of $0.13 \mathrm{~nm}$ respectively for B1 and B2 (Table 1).

When stellar light propagates through the atmosphere it is affected by refraction and by extinction (pure absorption + scattering). Normally these two effects can be distinguished, since refraction will affect mainly the direction of propagation and the global intensity, while extinction will mainly affect the shape of the remaining spectrum (in addition to the intensity). Some authors have classified occultations as refractive or absorptive (Smith and Hunten, 1990; Yee et al., 2002). Unfortunately the two effects are present together, because the index of refraction depends on the wavelength and it was recognized right from the beginning by the GOMOS proposers that scintillations 
(a refractive effect) would modify the spectrum of the star seen through the atmosphere, an effect detrimental to the accuracy of the ozone retrieval. Two fast photometers $(1 \mathrm{kHz}$ sampling) are included in GOMOS, which help to correct the effect of scintillations.

Given this instrument setup measuring $\mathrm{O}_{3}, \mathrm{O}_{2}$ and $\mathrm{H}_{2} \mathrm{O}$, it was also clear right from the beginning that additional measurements (besides ozone) could be made with GOMOS, some directly relevant to the ozone problem, and some others less directly related: the vertical distribution of $\mathrm{NO}_{2}, \mathrm{NO}_{3}$, aerosols, air, temperature, and turbulence parameters. The theoretical interest of the various species is discussed below, while actual GOMOS results are briefly reviewed in Sect. 7 .

\subsection{Ozone}

A transmission measurement is known to achieve its best accuracy with an optical thickness around 1 . The large dynamic rage of the ozone absorption cross-section extending from the UV to the visible (Fig. 2) offers the opportunity to probe the whole atmosphere from the mesosphere down to the upper troposphere (where there is still enough signal, Fig. 13). Obviously, the mesospheric ozone layer is probed around $250 \mathrm{~nm}$, where the ozone crosssection is at its peak, while at lower altitudes there is no more UV light transmitted, and the Chappuis band is used to determine the slant density of ozone. GOMOS provides routinely hundreds of vertical profiles per day (Fig. 14) of unprecedented accuracy distributed all over the globe, providing data to construct 3-D maps of ozone from the tropopause to the mesosphere.

In Sect. 7 a short overview (including papers of the GOMOS ACP special issue) is presented of what has been done on ozone with GOMOS to date. Here we discuss in more general terms several lines of research on ozone that may be performed with GOMOS.

Climatology of ozone: a study of all the data acquired in 2003 determined a new climatology of ozone (Kyrölä et al., 2006), supplementing the previous Fortuin and Kelder (1998) climatology, with an average value, and also an estimate of the natural variability of ozone as a function of altitude, season and latitude.

The understanding of photo-chemistry: in the upper stratosphere region (say, $35-50 \mathrm{~km}$ ), ozone is in photochemical equilibrium: the chemical lifetime of $\mathrm{O}_{3}$ (actually, $\mathrm{O}_{\mathrm{x}}=\mathrm{O}+\mathrm{O}_{3}$ ) is only $10^{4} \mathrm{~s}$ (Brasseur and Solomon, 1984). It is therefore an important region, where the comparison of actually measured ozone density may be compared to the predictions of a Chemistry Transport Model (CTM), in order to detect potential deficiencies in the chemistry prescribed in the models. Marchand et al. $(2004,2007)$ have carried out such an exercise, as summarized in Sect. 7, resulting in a suggestion of a significant revision of the temperature dependence of one constant, namely the reaction between ozone and $\mathrm{NO}_{2}$.
The study of time series and trends: though models are predicting that it will take several tens of years for ozone to recover pre-CFCs values, it is important to monitor precisely its behaviour, as a function of altitude and latitude. An estimate of the GOMOS ozone trend capabilities was made (Bertaux et al., 1991) by analogy with an earlier computation made by Frederick (1984). Frederick addressed the problem of the detection of ozone trend from one single ground station, performing measurements at regular intervals, $\Delta t$, sufficiently large that two consecutive measurements are completely de-correlated. Annual, semi-annual and quasibiennal variations were assumed to be regressed.

The actual GOMOS geographical coverage (see next section) is such that, with a particular star covering a circle of constant latitude, the measurement point comes back almost to the same longitude after 3 days (the duration of orbit of 100 min corresponds to $14+11 / 35$ orbit per day), which is about the decorrelation time observed for ozone over a particular ground station (2-3 days). Therefore, we may consider that all (or most) GOMOS ozone vertical profiles are indeed de-correlated.

Considering the case of 9 latitudes bands of $20^{\circ}$ each, Bertaux et al. (1991) found that the smallest ozone trend detectable by GOMOS would be $0.05 \%$ per year, assuming a precision of $\approx 5 \%$, and a natural variability of $4 \%$ at a given altitude (say, between 20 and $40 \mathrm{~km}$ ). Indeed, the achieved precision is better than 5\% on most stars observed, but the natural variability of ozone (at each altitude, season, latitude) was not well documented and this is something that can be achieved with GOMOS. Accurate determination of ozone density requires that the accuracy on a single measurement be smaller than the natural variability. The actual accuracy achieved by GOMOS is discussed in a later section; bright stars are preferred to do this exercise, which yield in principle an accuracy better than $1-2 \%$.

It must be noted however that the above formulation for deriving a linear trend assumes that all time variations are periodic and may be regressed. It is possibly not the case in reality, if volcanoes, solar activity or other sporadic factors are also playing a role in the ozone budget. For instance, the influence of Quasi Biennal Oscillation (QBO) on ozone was shown to depend on altitude: Hauchecorne et al. (2010) found three different regions with different influences. Also, the period of QBO is not fixed, and regression may be inaccurate. Therefore, the very concept of linear trend must be approached with caution. What remains true is that time variations of ozone are monitored with an unprecedented accuracy with GOMOS. In a companion paper of this $A C P$ special issue, Kyrölä et al. (2010) show some examples of time series of ozone at various altitudes. Above $20 \mathrm{~km}$ in the stratosphere, the median variability is found to be $5.5 \%$, while the median of the retrieval error is $0.6 \%$ (to be compared to the assumed above mean values of $4 \%$ and $5 \%$, respectively, to estimate the accuracy on the trend). 
Finally, it can be remarked that a higher precision on slant densities is obtained than on local densities, and all these primary data are also available in Level 2 products for trend studies.

Ozone as a tracer of dynamics: ozone has a long lifetime below $30 \mathrm{~km}$, and geographical variations of ozone density can be used as a tracer of winds in the stratosphere. Therefore, there is an interest to assimilate ozone densities at altitudes below $30 \mathrm{~km}$ in GCM meteorological models. Of course, Near Real Time products are needed for such a purpose; ESA is delivering GOMOS ozone data within $3 \mathrm{~h}$ of collection through the Ground Segment system. However, it is not yet assimilated routinely in GCM models.

Day-side measurements of ozone: for a complete description of the ozone field, it was felt necessary to characterize accurately the diurnal variation of ozone. Its amplitude increases from a few percent at $50 \mathrm{~km}$ to $80 \%$ at $70 \mathrm{~km}$, which reflects mainly variations in the diurnal equilibrium between $\mathrm{O}_{3}$ and $\mathrm{O}$. Below about $65 \mathrm{~km}$ altitude, almost all the $\mathrm{O}$ is converted rapidly to $\mathrm{O}_{3}$ after sunset. A study of ozone trend would be incomplete if only night side measurements would be collected, and some efforts were made in the design of GOMOS in order to collect at least a significant number of vertical profiles of ozone also on the day-side.

The day-side situation is quite challenging for the stellar occultation technique: the star signal is competing with the limb background radiance of the sun-illuminated atmosphere, due to Rayleigh and aerosol scattering. This requirement is a design driver: while the spectrometers need no narrow entrance slit for the star spectrum to be recorded with a high resolution, and could have a large aperture, the rejection of undesired limb radiance calls for a small solid angle of the entrance aperture, which in turn calls for a good tracking accuracy, in order to get the star image at the focus of the telescope (which is also the entrance aperture of the spectrometers) always fully in the aperture. Its width was taken as equivalent to $195 \mu \mathrm{rad}$, or 10 spectrometer pixels. As we shall see, tracking performances of GOMOS are such that many stars can be tracked down to $25 \mathrm{~km}$ on the dayside.

In addition, the entrance slit aperture is elongated in the vertical direction (perpendicular to the limb), which enables simultaneous measurement of the spectrum of the limb radiance above and below the star, in order to subtract it to obtain the pure star's signal. Three bands of 7 CCDs lines each are therefore devoted to the measurements of the 3 spectra. This is also useful in some nighttime conditions in order to subtract some natural atmospheric emissions.

Mesospheric ozone: with respect to PMCs, the chemistry of the mesosphere is also addressed, because GOMOS is able to measure ozone concentration in the mesosphere by absorption at $250 \mathrm{~nm}$, where the $\mathrm{O}_{3}$ cross-section is maximum (Fig. 2). In this altitude range (80-100 km) ozone is produced by reaction of $\mathrm{O}$ with $\mathrm{O}_{2}$, atoms of $\mathrm{O}$ coming themselves from the photo-dissociation of $\mathrm{O}_{2}$ by solar $\mathrm{UV}$ in the day-side thermosphere. Ozone is destroyed by reactions with hydrogen radicals, i.e. $\mathrm{H}, \mathrm{OH}$, and $\mathrm{HO}_{2}$. Indeed, the high vertical resolution of GOMOS (Fig. 14) has enabled us to show that there is an enormous sink of ozone around $80 \mathrm{~km}$ altitude, where the ozone concentration almost vanishes: another type of "ozone hole", that would deserve a dedicated model study, in conjunction with GOMOS ozone profiles.

The photochemical lifetimes of oxygen and hydrogen radicals in the mesosphere are only a few hours (Brasseur and Solomon, 2005), and the ozone concentration should reflect a rapid photochemical equilibrium. In spite of this relatively simple situation, there are some significant discrepancies between observations (relatively scarce before GOMOS) and the prediction of current modeling. The numerous ozone profiles obtained by GOMOS support further documentation of this discrepancy, which would help to improve the modeling. At the time of the GOMOS proposal (1988) we thought that if we would not be able to reproduce mesospheric ozone through modeling of the mesosphere where the ozone balance is relatively simple, it would cast serious doubts about the modeling of ozone in lower regions of the atmosphere, where the same reactions (among others) do occur also when oxygen and hydrogen radicals are present. One powerful diagnostic to validate models is the time variations of mesospheric ozone, and in particular the day-to-night variation, and variations associated with solar activity. It turns out that there are still discrepancies between model and observations (Sect. 7.2), and the mesospheric ozone balance is certainly not as simple as once believed, probably due to atmospheric dynamic factors rather than chemical factors.

Not expected during the design of GOMOS, was the Tertiary Ozone Maximum (TOM, Fig. 28), first reported by Marsh et al. (2001); GOMOS observations of the TOM were reported by Sofieva et al. (2004, 2009), and are briefly reviewed in Sect. 7.3.

As a conclusion concerning the ozone objectives of GOMOS, it is clear that not only must we measure as well as possible the ozone 3-D field and its time variations (to check the results of CFCs release limitations), but we must also understand the detailed underlying mechanisms governing the ozone budget, in particular by comparison with chemical models, or by assimilation into Chemistry Transport Models (CTM). In this respect measurements of a number of other atmospheric parameters that are important for the ozone budget are also included in the design of GOMOS, as seen further below. Needless to say, the measurements carried out simultaneously by MIPAS and SCIAMACHY on ENVISAT are also useful for the understanding of mechanisms controlling ozone. Clearly, the degree of our understanding impacts directly our capability to predict accurately (through modeling) what will be the future behaviour of ozone on the long term (tens of years), and this is our ultimate goal. 


\subsection{Nitrogen gases $\mathrm{NO}_{2}$ and $\mathrm{NO}_{3}$}

Both gases participate in the $\mathrm{NO}_{\mathrm{x}}$ family, involved in a catalytic cycle that provides the major loss processes for odd oxygen $\left(\mathrm{O}_{3}\right.$ and $\left.\mathrm{O}\right)$ in the stratosphere (Crutzen, 1971; Brasseur and Solomon, 2005). $\mathrm{NO}_{2}$ has an extended absorption band in the visible, with conspicuous peaks at $430-450 \mathrm{~nm}$ (Fig. 2), and its stratospheric quantity is enough to be measured in the transmission spectrum of the atmosphere (Fig. 13). $\mathrm{NO}_{3}$ is rapidly photo-dissociated and does not exist during the day. It can be observed only during the night, thanks to its strong spectral signatures at 617 and $655 \mathrm{~nm}$. It has no emission in the IR, and GOMOS is providing unique routine observations of $\mathrm{NO}_{3}$. SCIAMACHY has also measured $\mathrm{NO}_{3}$ occasionally, by performing moon occultations (Amekdudzi et al., 2009).

Other gases interesting for the ozone budget have also some absorption in the wavelength covered by GOMOS, like $\mathrm{OClO}$, and $\mathrm{BrO}$. However, in view of the weak stellar signal, and the scarcity of these absorbers, one cannot hope to measure them in one single occultation. There is more hope when piling up many transmission measurements to increase significantly the signal-to-noise ratio, as was done successfully by Fussen et al. (2006) and Tétard et al. (2010) for $\mathrm{OClO}$ and is summarized in Sect. 7.8.

\subsection{Temperature and oxygen}

There is an important wavelength domain $(310-350 \mathrm{~nm}$, the Huggins bands) where the $\mathrm{O}_{3}$ cross-sections are strongly dependent on the atmospheric temperature. Therefore, a long-term variation of absorption in the UV as measured by GOMOS might be due to temperature variation and wrongly attributed to an ozone density variation. It was felt necessary to include a temperature measurement within GOMOS. This is possible in principle with the $\mathrm{A}$ band of $\mathrm{O}_{2}$ at $760 \mathrm{~nm}$ (Fig. 15). Since $\mathrm{O}_{2}$ is a perfectly mixed gas and the air is in hydrostatic equilibrium, its scale height, $H$, is directly connected to the atmospheric temperature, $T$, whose vertical profile may be retrieved from integration of the hydrostatic equation:

$$
\frac{1}{T} \frac{d T}{d z}+\frac{1}{\left[\mathrm{O}_{2}\right]} \frac{d\left[\mathrm{O}_{2}\right]}{d z}=-\frac{\mathrm{mg}}{k T}=-\frac{1}{H}
$$

This measurement of $\mathrm{O}_{2}$ also allows relating all the measurements of ozone density (and other species) to the air density, thus yielding the mixing ratio $\left[\mathrm{O}_{3}\right] /[$ air $]$, which is often a quantity used in atmospheric models: comparison of GOMOS measurements with models may be easier using mixing ratios than absolute densities.

The ILAS-I and ILAS-II instruments flown on Japanese missions ADEOS-1 and ADEOS-2 has been performing similar measurements of $\mathrm{O}_{2}$ in the $\mathrm{A}$ band by solar occultation, with a somewhat similar spectral resolution, but a better signal-to-noise ratio. However, their interpretation suffered from uncertainties on the LOS altitude retrieval, associated with pointing uncertainties on the extended solar disk. The GOMOS $\mathrm{O}_{2}$ results will be discussed in Sect. 7.

\subsection{Water vapor $\mathrm{H}_{2} \mathrm{O}$}

Water vapor is injected into the stratosphere in tropical regions, and is also created by the oxidation of methane in the upper stratosphere. Water vapour plays an important role in the photo-chemistry, and in particular is the major scavenger of ozone (by $\mathrm{HO}_{\mathrm{x}}$ catalytic reaction cycle) at altitudes greater than $40 \mathrm{~km}$ (Brasseur and Solomon, 2005). The strongest absorption band of $\mathrm{H}_{2} \mathrm{O}$ in the silicon CCD domain is in the Near Infra Red (NIR), around $936 \mathrm{~nm}$ (Fig. 16). Although this wavelength region is in principle free of other (known) stratospheric gaseous absorbers, we have adopted a relatively high resolution for spectrometer B2, because it was already present of spectrometer B1 anyway.

In principle MIPAS, looking at the far infra-red emission of water vapour, is more sensitive than GOMOS for water vapour. However, GOMOS may also provide useful $\mathrm{H}_{2} \mathrm{O}$ measurements for two reasons. In the far IR, an absolute radiometric calibration of the instrument is necessary to retrieve $\mathrm{H}_{2} \mathrm{O}$ from MIPAS, which may be detrimental for minute trend detection. Also, around the Upper Troposphere Lower Stratosphere (UTLS), the region of exchange of water vapour between the troposphere and the stratosphere, the vertical gradient of $\mathrm{H}_{2} \mathrm{O}$ is very large, in which case the perfectly well determined altitude range of each GOMOS transmission measurements is a very important asset. In particular, it is hoped that GOMOS could contribute to discriminating between various proposed mechanisms to explain the paucity of $\mathrm{H}_{2} \mathrm{O}$ in the stratosphere: injection only above tropical convective cumulo-nimbus clouds extending at high altitudes, and therefore very cold and trapping $\mathrm{H}_{2} \mathrm{O}$ by condensation, or further dessication in the lower stratosphere by a series of cooling episodes (and condensation and sedimentation) with formation of sub-visible clouds, possibly forced by gravity waves. These clouds are indeed observed in GOMOS aerosol data (Sect. 7, and Vanhellemont et al., 2005). The high vertical resolution allows measurement just above thick tropospheric clouds, while emission instruments would be contaminated by the clouds in their FOV.

At mid-latitudes, $\mathrm{H}_{2} \mathrm{O}$ may be injected into the stratosphere from lateral motions of air masses along isentropic trajectories.

A very important long term objective is also related to Global Warming, which may result in a drastic change (increase or decrease) of water vapour injection in the stratosphere, with a potential impact on ozone, in particular in the upper stratosphere. Balloon-borne dew-point hygrometers flown near Boulder, Colorado, show a positive trend (increase) of $+0.03 \mathrm{ppmv} \mathrm{yr}^{-1}$ at $25 \mathrm{~km}_{\text {over the period }}$ 1992-2005, and no variation at $16 \mathrm{~km}$, while HALOE solar 


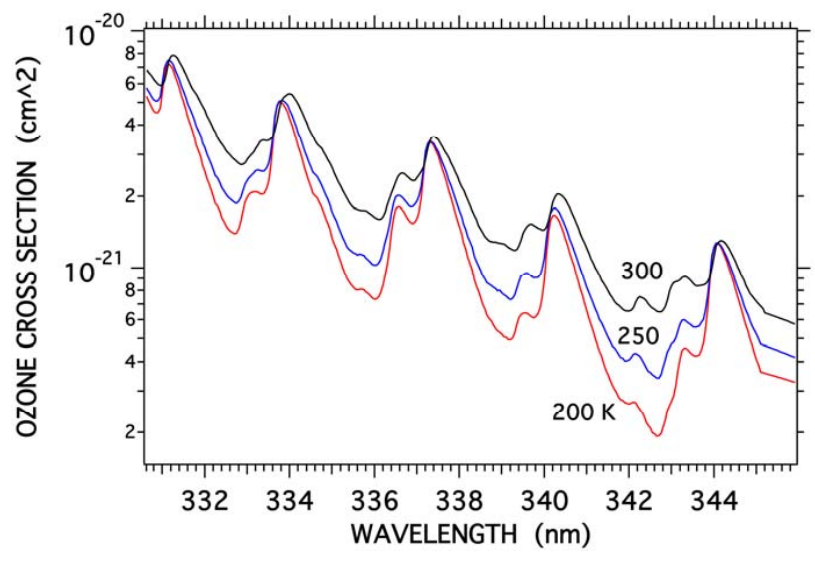

Fig. 3. Absorption cross-sections of ozone at 200, 250 and $300 \mathrm{~K}$ in the Huggins bands. In principle, observations of actual transmission spectra should enable retrieval of a temperature estimate.

occultation instrument on board UARS showed no variation at $25 \mathrm{~km}$ and a negative trend of $-0.04 \mathrm{ppmv} \mathrm{yr}^{-1}$ at $16 \mathrm{~km}$. Thus, it is desirable to have other long time series from instruments operating now, with some overlap with the previous period 1992-2005, which is exactly what GOMOS on ENVISAT may provide in the lower stratosphere (LT) and upper troposphere (UT). In fact, due to the extreme sensitivity of $\mathrm{H}_{2} \mathrm{O}$ abundance to atmospheric temperature at the hygropause, GOMOS might be able to detect trends on this temperature based on a potentially observed $\mathrm{H}_{2} \mathrm{O}$ trend. At any rate, since $\mathrm{H}_{2} \mathrm{O}$ and its derivative $\mathrm{HO}_{\mathrm{x}}$ radicals are scavengers of ozone, the accurate monitoring of $\mathrm{H}_{2} \mathrm{O}$ is also important to understanding the future of ozone balance.

\subsection{Temperature and air density, and ECMWF data}

Besides the measurement of $\mathrm{O}_{2}$ vertical profile and derivation of temperature, there are several other methods which have been thought of to retrieve the vertical profile of temperature from GOMOS data, each of them in a somewhat limited range of altitude:

- The time delay between blue and red scintillations is a totally new method, and allows determining a High Resolution Temperature profile, HRTP (see Sect. 7.15, and Dalaudier et al., 2006).

- Rayleigh extinction depends on the air density vertical profile, which is retrieved from Spectrometer A (UVVis), and then the temperature may be retrieved with the hydrostatic equation, as in the case of $\mathrm{O}_{2}$. However, the accuracy actually achieved for air density is not good enough to derive a useful air density and associated temperature.

- As noted previously, the ozone cross-section is sensitive to temperature in the Huggins bands' wavelength range
$(310-350 \mathrm{~nm})$ (Fig. 3). GOMOS has enough spectral resolution to make a fit of a temperature-dependent model transmission, which should allow extraction of an estimate of the temperature when absorption in this range $(310-350 \mathrm{~nm})$ is moderate, which corresponds to the $25-50 \mathrm{~km}$ altitude range with typical ozone profiles (Fig. 12).

- The dilution factor depends on the atmospheric profile, and its measurement (by averaging scintillations) may be inverted to yield a density and temperature profile. However, absorption and scattering must also be known precisely.

- The bending angle of refraction is the angle between the apparent direction of the star seen from ENVISAT and the absolute direction in the sky. It may be retrieved from the telemetered angular position of the SFA (Steering Front Assembly) plane mirror, which follows the apparent direction of the star, thanks to GOMOS' active tracking system. One must take into account the actual attitude of ENVISAT as a function of time during the occultation. This is similar to the method used with the imager of MSX/UVISI (Yee et al., 2002). However, some attempts to do this have shown that the accuracy of the SFA angle measurements was too coarse to contribute to temperature profile retrieval.

- On the day-side, there is some Rayleigh scattering emission of solar photons by air, which can also be inverted to yield a vertical profile of air, as well as another temperature profile. One difficulty is that there is a contribution from the nadir brightness to the scattered light. It is in principle possible to discriminate the direct solar component from the indirect nadir contribution in the scattered emission in the stratosphere, because of some strong tropospheric absorptions band imprinted on the light coming from the nadir. The $\mathrm{O}_{2}$ band is not suitable because there is also some emission around $760 \mathrm{~nm}$ and the $\mathrm{H}_{2} \mathrm{O}$ band at $936 \mathrm{~nm}$ is possibly the best indicator of the nadir contribution. In fact, even if there is a constant error factor in the determination of the air density because of an incorrect estimate of the nadir contribution, it is the vertical gradient that is used in the integration of the hydrostatic equilibrium equation, and inasmuch as the nadir intensity may be regarded as constant during on day side occultation, it should not affect the temperature retrieval.

At the present time (2009), only the HRTP method, using the time delay between the two fast photometers has been developed and validated. It will be implemented in the next version of the operational software, resulting in a GOMOS temperature profile in the Level 2 Product. It was found important to include also in the Level 2 
product the air density and temperature profiles derived from the ECMWF (European Centre for Medium-range Weather Forecasts) analysis, because it is more accurate than the GOMOS temperature/air density profile on most occasions. It also facilitates a systematic comparison of GOMOS temperature/density data with ECMWF profiles serving as a reference. The users of Level 2 GOMOS data should be cautious about the possible confusion of the ECMWF data, contained in the Level 2 GOMOS product, and the genuine GOMOS data. It should be noted that the Level 2 processing makes use of the ECMWF field of air density and temperature (analysis) in order to compute an accurate ray tracing of the LOS through the atmosphere with refraction, and also to prescribe the air line density for extinction in the spectral inversion of each transmission spectrum.

Contained in the Level 2 GOMOS product, the $\mathrm{O}_{2}$ line density and temperature retrieval suffered from earlier incorrect cross sections from the HITRAN 2000 data base (leading to up to $20 \%$ deviation from ECMWF data). Though they were improved in the more recent HITRAN 2004 release we do not feel confident enough yet to use the $\mathrm{O}_{2}$ measurements for a temperature retrieval in the Level 2 product as discussed in Sect. 7.

\subsection{Turbulence and scintillations}

The vertical transport of chemical species is of fundamental importance for monitoring ozone and other trace gases in the stratosphere. In 1-D models, it is most often parameterized by an eddy diffusion coefficient $K_{z z}$ associated with turbulence. Therefore, the inclusion of fast photometers in the design of GOMOS was not only in order to correct the spectra for scintillations, but also to study the "climatology" of turbulence, and associated vertical transport. However, it should be recognized that global 3-D Chemistry Transport Models ignore such a parameter and vertical transport is calculated only by advection, with a vertical velocity resulting from the divergence of the horizontal flux in a cell.

\subsection{Aerosols}

Stratospheric aerosols are mainly produced by volcanic eruptions, and transformation of $\mathrm{SO}_{2}$ into sulphuric acid droplets. The content depends therefore on the history of volcanic eruptions. Since these particles may participate in heterogeneous chemistry processes, it is important to monitor their quantity and to try to determine their composition and size distribution. At the time of the ENVISAT launch, the aerosol content at $20 \mathrm{~km}$ was possibly at a historical record low, with some increase at the end of 2002 (Thomason and Taha, 2003). This is a situation rather favourable for stellar occultation measurements of other species in the lower stratosphere. It can be noted that both the extinction by the aerosols and their scattering properties may be obtained with GOMOS, by combining night measurements (extinction) and day side measurements (scattering) for a better retrieval of physical properties of the aerosols. This work is still to be done.

\subsection{Polar Stratospheric Clouds (PSC)}

PSCs are high altitude clouds encountered during very cold conditions in polar regions. They are of several types, according to the nature of the condensate, besides $\mathrm{H}_{2} \mathrm{O}$. Before launch, we thought that their horizontal optical thickness would be so large that the stellar signal would be lost by the star tracker. In such a case the main information would therefore be the altitude at which a very sudden loss of the star signal by the star tracker is observed. It turned out that in many cases, the star may be seen through the PSC, as discussed in Sect. 7, and in Vanhellemont et al. (2005, 2010).

\subsection{Polar Mesospheric Clouds (PMC)}

The polar summer mesosphere is characterized by extremely low temperatures (below $150 \mathrm{~K}$, the coldest region of the entire planet), due to a strong upward motion and subsequent adiabatic cooling of air. In the altitude range between $\sim 80$ and $90 \mathrm{~km}$, water vapour abundance becomes higher than the local saturation vapor pressure, and icy particles condensate around condensation nuclei, which could be either meteorite dust particles or large water cluster ions. Very small ice particles are electrically charged and lead to measurable signatures in the form of strong radar echoes, called Polar Mesosphere Summer Echoes (PMSE). Under favourable conditions, these small icy particles grow and sediment. They sublimate when they reach warmer layers below the cloud. Therefore, smaller particles are found at the top and larger particle towards the bottom. The largest of them can be observed by optical techniques and are then called Polar Mesospheric Clouds (PMC) when observed from space, or Noctilucent Clouds (NLC) when observed from the ground at twilight. This part of the mesospheric ice layer is a thin layer $(\sim 2 \mathrm{~km})$ of small ice particles $(r<0.1 \mu \mathrm{m})$ at $83-85 \mathrm{~km}$ of altitude, whose vertical optical thickness, $\tau_{\mathrm{v}}$, is $\sim 10^{-4}$, and tangential optical thickness, $\tau_{\mathrm{h}}$, around $10^{-2}$.

These ice particles produce scattered sunlight which is detected by the GOMOS spectrometers and photometers (Fig. 45). The actual brightness is about the same as Rayleigh scattering at $50 \mathrm{~km}$ of altitude and gives a significant signal that can be interpreted in terms of Mie theory and particle size and nature. In absorption, the $1 \%$ absorption over $2 \mathrm{~km}$ of altitude is difficult to measure.

In his review of the subject, Thomas (1991) advocates that the appearance of PMC is a strong evidence of Global Change. The explanation would be that methane has been increasing steadily since 1850 (due to growing population and associated development of agriculture and livestock), with a growth rate of $1 \%$ per year (this growth rate has declined almost to zero in more recent years). Indeed, 
oxidation of methane $\left(\mathrm{CH}_{4}\right)$ is an important source of $\mathrm{H}_{2} \mathrm{O}$ above $\sim 50 \mathrm{~km}$, and therefore an increase of $\mathrm{CH}_{4}$ at ground level will reflect some years later as an increase of $\mathrm{H}_{2} \mathrm{O}$ at mesopause level and a higher probability of encountering frost point temperature conditions. The saturation vapour pressure decreases by a factor of 5 for a decrease of only $4 \mathrm{~K}$ for summer mesopause conditions. Therefore, even a moderate climatic temperature change (as induced for instance by increased $\mathrm{CO}_{2}$ cooling at these altitudes) could result in a larger PMC coverage over the globe. With its capability to detect the presence of PMCs at each occultation, GOMOS will enable creation of a good corpus of data over the whole globe. Of particular interest will be documenting the North-South asymmetry, and to monitor the evolution of PMC cloud cover and the location of its equator boundary. GOMOS detects easily PMCs in the fast photometer signal, as described in Pérot et al. (2010) and in Sect. 7.12.

\subsection{Day-side limb scattering measurements}

On the day-side, the limb radiance (background) due to Rayleigh and aerosol scattering of solar light can also be used to retrieve ozone and other gases, with a technique used by OSIRIS on ODIN (Tukiainen et al., 2008), and SCIAMACHY on ENVISAT (Bovensmann, 1999). The spectrum of the recorded light is influenced by ozone absorption (and also other gases) and may be analysed in terms of ozone (and other gases) vertical profile. Though the retrieval algorithm is much more complex than the occultation one, it will be useful to compare values of ozone retrieved by the two techniques. Included in GOMOS design is the capability to perform a "dummy" occultation, in which the tracking mirror is no longer piloted by the image of a star in the star tracker, but rather programmed to perform a motion as if there were a star to be occulted, sweeping the whole range of altitudes across the bright limb. In this case, there is no star signal, and only the bright limb emission is present (with some stray light contamination). However, there is one advantage to analysing the limb radiance during a star occultation: the observed altitude is well known, while the altitude accuracy is less when there is no star. New algorithms are presently being developed to retrieve ozone and other constituents from GOMOS bright limb measurements, sponsored by ESA and led by Finnish Meteorological Institute.

\subsection{Moon and planets}

Like stars, planets may be used as targets for performing occultations. The advantages are a somewhat larger intensity and also less scintillation effects, due to the nature of an extended source. The spectral resolution is degraded because the entrance slit of the spectrometer is much wider than the image of the star. Therefore, with the moon or a planet, which are extended objects, the actual spectral resolution is dictated by the angular size of a planet, if smaller than the slit, or by the size of the slit (corresponding to 10 pixels) for the moon. If the disc is too large, then it is not possible to track it properly with the GOMOS star tracker, as we found out. This is the case for Jupiter and Venus, but for Mars it worked very well. A particularly attractive opportunity was the opposition of Mars around August 2002, when it was at its shortest distance from the Earth since some time before the beginning of the space age. Since the absolute calibration of GOMOS is achieved in flight with a high accuracy by comparison with standard stars, the albedo of planets can be measured also from space with a great accuracy over the entire UV-Vis-NIR range of the GOMOS spectrometers.

Using the moon is also in principle an attractive proposition, because of the high flux expected. However, it cannot be tracked with the star tracker; therefore, GOMOS would have to be operated in the "dummy" mode of occultation, but then refraction will make the moon moving in the FOV. Calculations show that one cannot keep the moon always in the FOV with a fixed inertial direction of the LOS. Moon occultations have not been attempted up to now.

\section{Instrument description, operations and coverage, typical observation results}

\subsection{Instrument description}

\subsubsection{Measurement principle and overall instrument configuration}

GOMOS is an instrument designed to measure the atmospheric transmission at the limb in the ultraviolet, visible and near infrared with a good spectral resolution, in order to identify by their spectral signatures atmospheric ozone and other trace gases. It exploits a stellar occultation technique, whose measurement principle is described in Fig. 1. The spectral flux of a pre-selected star is measured continuously during the setting of the star behind the earth due to the motion of the spacecraft on its orbit.

When the LOS to the star is fully outside the atmosphere (i.e. tangent height $z$ of the LOS higher than $110 \mathrm{~km}$ ), the unattenuated star spectrum $\operatorname{So}(\lambda)$ (Eq. 1) is measured. Typically the ten first spectra are averaged together to give the Reference star spectrum, So $(\lambda)$. Then, during the whole occultation, while the star sets behind the horizon (which last from 30 to $250 \mathrm{~s}$ ) and the tangent height $z$ is decreasing, the occulted spectra $S(\lambda, z)$ are measured every $0.5 \mathrm{~s}$ with no dead time. These spectra are divided by the reference spectrum of the target star So $(\lambda)$, enabling the acquisition of spatially high-resolution atmospheric transmission spectra $T_{m}(\lambda, z)$. Using these measured horizontal transmission spectra, $T_{m}(\lambda, z)$, and the associated known cross-sections for each chemical species, the horizontal and vertical density profiles are retrieved, as briefly described in the Introduction and in Sect. 5. Typical reference spectra for the various stars are shown on Fig. 11. 
ENVISAT is nadir oriented; a given star has a constant inertial direction, and therefore its direction in the ENVISAT mechanical frame of reference is changing. Therefore, GOMOS needs a pointing system to track the star continuously. It is composed of one plane mirror, actuated by a Steering Front mechanism and piloted by a Star Acquisition and Tracking system (SATU), as shown on Fig. 4. The star light is sent to a Cassegrain mirror telescope, whose area is divided into two parts coated differently, one devoted to the UV-Vis, the other to the near IR. Light is focused onto a slit, separated by dichroïc plates into various beams and sent to the UV-Vis spectrometers A1 and A2, spectrometers B1 and B2, fast photometers and the SATUs (prime and redundant).

The two fast photometers $(1 \mathrm{kHz}$ sampling rate $)$ were implemented to correct the measured transmission spectra for the adverse effect of scintillation, and also to determine important atmospheric parameters describing the state of turbulence of the stratosphere.

Given the constraints of the ENVISAT platform, and the scientific specifications defined by the SAG group, the Astrium company, with the help of a number of European companies, designed, developed, calibrated and delivered (under the responsibility of ESA) the GOMOS instrument, with a mass of $166 \mathrm{~kg}$, power consumption of $146 \mathrm{w}$, and a constant data rate of $222 \mathrm{~kb} \mathrm{~s}^{-1}$. Although GOMOS is specifically optimized for night observations, it performs also measurements during the day (bright limb conditions).

By its principle, GOMOS is inherently a self-calibrating instrument. This means that long-term drifts in the instrument's radiometric response are in theory compensated since only the ratio of measurements acquired in a relatively short time interval (of the order of $1 \mathrm{~min}$ ) are used to compute transmission spectra. In such short intervals, changes in the instrument response are negligible. However, if the radiometric sensitivity is decreasing significantly, it will hamper the quality of the data (due to increase of the error bars). This has not happened with GOMOS up to now. In addition, some other long-term drifts may also introduce larger error bars, and even a bias, if they are not taken into account properly. This is the case, for instance, of the large increase of the Dark Charge of the detectors. It was supposed to be a negligible correction to the signal, but it turned out that the EEV CCD detectors have seen their dark charge increase dramatically to such a point that the DC subtraction algorithm must be accurate, and even in this case, the error is increasing for dim stars as discussed at the end of Sect. 4.1.

\subsubsection{Pointing function description}

As seen from ENVISAT, stars are setting in the hemisphere opposite to the velocity vector. Therefore, GOMOS is placed near one end of ENVISAT, to look backwards. A frame of reference is defined with $+\mathrm{z}$-axis towards nadir, and
$\mathrm{XZ}$ plane maintained in the orbital plane (except for the yaw steering law to compensate for the Earth's rotation for imaging instruments). One particular pointing direction is defined by two angles: the azimuth, AZ (measured from the orbital plane, $0^{\circ}$ looking opposite to the velocity vector), and the elevation, EL, measured from the nadir direction. The limb is around $63^{\circ}$, which is $27^{\circ}$ below the horizontal plane.

A Steering Front Mirror is used to orientate the LOS towards pre-selected stars. This plane mirror enables the detection and tracking of a star over a wide angular range $\left(100^{\circ}\right.$ in azimuth, from $-10^{\circ}$ to $+90^{\circ}$, and $8^{\circ}$ in elevation). The instrument line-of-sight geometry covers both rearward (anti-flight direction) and sideways viewing directions. However, when ENVISAT is near the South Pole, the sun is in the rear hemisphere. A baffle protects the entrance of GOMOS, but it occults most of the hemisphere on one side of the orbital plane, limiting the azimuth range on this side to $-10^{\circ}$.

The target star is detected by the Star Tracker, a CCD covering a field of view of $0.6^{\circ}$, with a focal length of $0.63 \mathrm{~m}$. After detection, the star image is moved to the centre of the CCD and another algorithm performs the tracking with a very high accuracy. The pointing application software realizes the measurement of the position of the star on the CCD with a background removal. It then commands the Steering Mechanism in closed loop to keep the star image at the centre of the CCD during the occultation. Towards the end of an occultation sequence, the star light becomes eventually so attenuated that the instrument can no longer observe and track it. The star is finally lost behind the horizon and the instrument continues by moving its pointing direction (i.e. the mirror) toward the next star according to a pre-defined observation plan. In this way the instrument may acquire up to 40 stars per orbit, yielding typically $\sim 100000$ occultation per year.

To achieve this very high pointing accuracy, an innovative concept has been developed: telescope, focal plane optics and detection units are fixed with respect to the host spacecraft structure; by moving the steering mirror implemented in front of the telescope entrance, the GOMOS line of sight is aligned with the star direction. The GOMOS pointing function actively controls the disturbances induced by the host spacecraft within a low bandwidth $(4 \mathrm{~Hz})$. This fine-pointing control loop also corrects for short-term perturbations as induced by the atmospheric refraction and a part of the scintillation effects. Outside the control bandwidth, the LOS stability is guaranteed by the limitation of structural amplification of micro-vibrations through the Steering Front Mechanism on one side, and Telescope and Optical Bench on the other side.

The Steering Front Mechanism (SFM, Fig. 5) design is optimized with respect to the mission needs: wide angular range in rallying (when the pointing mechanism is moved toward a new star to be occulted); high linearity (5\%) and low noise $(<20 \mu \mathrm{Nm})$ actuator in acquisition/centring/tracking. 


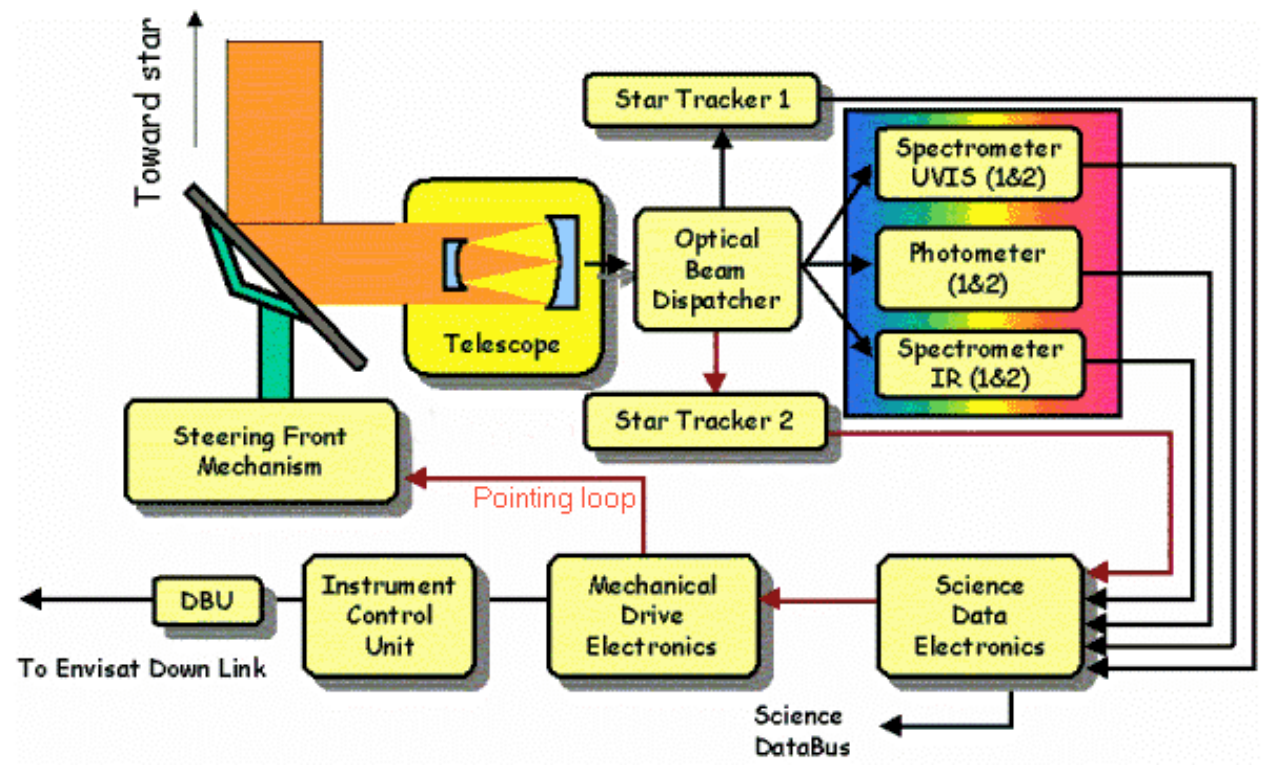

Fig. 4. GOMOS Instrument block diagram.

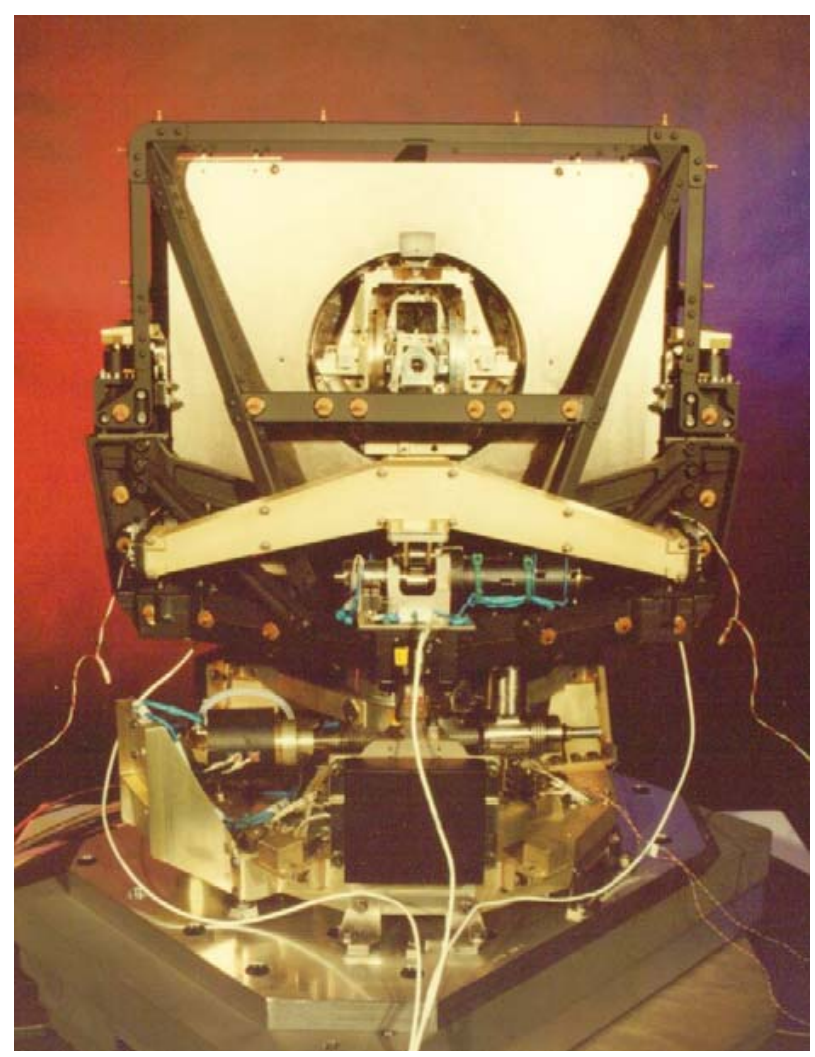

Fig. 5. Steering Front Mechanism rear view. It moves the plane mirror in order to keep the image of the star always in the entrance slit of the spectrometers.
These requirements led to a two-stage concept: a two-axis fine stage supported by a one-axis coarse stage (azimuth only).

The micro dynamical behaviour of the SFM was characterized on ground by tests performed on a fully representative breadboard. A complete functional validation of the pointing function (acquisition and tracking) has been performed on equipment breadboards with laser simulation. The number of coarse mechanism displacements is qualified for a 7-year lifetime.

Once a given star is acquired by the SATU, the SFM is given a nominal angular velocity corresponding to the predicted motion of the star as seen from ENVISAT (sent from ground by TC). The tracking system then computes only small corrections to this motion. However, at low altitudes when refraction bends the LOS to the star upward (w.r.t. the horizon), the correction becomes more important. With no correction, the star would go away from the CCD nominal reading zone, an effect noted with MSX mission, which was pointed inertially (Yee et al., 2002).

The pointing system is also able to track the star in spite of the additional apparent motion due to scintillations, up to a certain extent. In the case of strong scintillations, the tracking may be lost before the obscuration of the star by clouds at the limb.

It is to be noted that because of refraction, the apparent angular velocity of the star decreases with decreasing altitude. This is taken care of by the SATU; but an obvious improvement of the GOMOS design would be to pre-program a curve of angular velocity as a function of elevation angle, to compensate for refraction in a typical atmospheric profile. 
The final performances in flight are completely in line with the expected. The minimum star already tracked is fainter than magnitude 4 and the total number of stars which can be detected is more than 500. However, measurements were performed routinely on about 150 stars only. The rallying accuracy is better than $0.03^{\circ}$. More than $97 \%$ of the targets are detected and tracked successfully. The noise in tracking is less than $1 \mu \mathrm{rad}(1 \sigma)$ for the faint star in dark limb and $3 \mu \mathrm{rad}$ in bright limb. The robustness of the pointing chain allows tracking stars sometimes down to $5 \mathrm{~km}$ altitude; at other times, clouds or strong scintillations stop the tracking at higher altitudes, as shown in the histograms of Fig. 10.

\subsubsection{Spectrometers description}

The instrument's optical design is based on a $30 \mathrm{~cm}$ telescope ( $1.05 \mathrm{~m}$ focal length) that simultaneously feeds a medium resolution UV-VIS spectrometer, a high resolution IR spectrometer, two fast photometers and two redundant star trackers (see Fig. 4). The splitting of the entrance flux is done by the Optical Beam Dispatcher, placed at the telescope focal plane. Since for stellar spectrometry there is no need for a slit to limit the spectral resolution, a slit is placed in the OBD only to limit the background and stray light flux. But this implies that the stability of the image on the spectrometer has to be guaranteed by the tracking. Taking into account the very good pointing performance, the spectral stability is better than 0.1 pixel.

All the detection units are implemented on a thermally controlled optical bench. This bench and the telescope are mounted together and fixed on the instrument interface via carbon-fiber struts. The spectral dispersion is performed by two different gratings. The UVIS grating is an aberrationcorrected holographic grating. It is used at its first order. The light is then imaged on two CCDs (UV and VIS), which enables partial compensation for the grating's residual curvature. The IR grating is used in a Littrow configuration at high orders of diffraction (orders 13 and 16). After a dichroïc splitting, the light is then imaged on two CCDs (IR1 and IR2). The unused orders of diffraction are rejected by narrow band-pass filters located in front of the detectors.

The same type of detector is used for the 4 spectrometer channels. The CCD (from EEV) is a $27 \mathrm{~mm}$ length matrix of $1353 \times 143$ pixels. The pixel size is $20 \times 27 \mu \mathrm{m}$. They are thinned and back-side illuminated in order to improve their efficiency in the UV.

They can be used in different modes. In the normal mode (Fig. 6), 3 sets of lines are binned "on chip" to give 3 values per column. This gives 3 spectra. The central one is used to collect all the star target flux. The 2 others (above and below) are used for the dark signal and background correction. Each of these spectra is the integration over 7 lines (this parameter is programmable) equivalent to $0.01^{\circ}$. The total number of spectral elements is 2336 , which can be shared by programming between the 4 spectrometers. The baseline configuration is as follows:

$$
\begin{aligned}
& \text { spectro A1 : pixel } 1 \text { to } 450 \text { (248.14 to } 389.18 \mathrm{~nm}) \text {, } \\
& \text { spectro A2 : pixel } 451 \text { to } 1416(389.52 \text { to } 690.49 \mathrm{~nm}) \text {, } \\
& \text { spectro B1 : pixel } 1417 \text { to } 1836(755.12 \text { to } 774.59 \mathrm{~nm}) \text {, } \\
& \text { spectro B2 : pixel } 1837 \text { to } 2336(925.97 \text { to } 954.42 \mathrm{~nm}) \text {. }
\end{aligned}
$$

Three other modes, for which the integration time can be changed or the individual pixel data can be retrieved, are used for the calibration. The radiometric performance of these CCDs in terms of noise and quantum efficiency allows achieving a signal-to-noise ratio of the order of 20 in VIS and 6 in IR for stars of magnitude 3 (per pixel). However, an unexpected sensitivity of the CCD dark signal to the protons of the South Atlantic Anomaly leads to a high non-uniformity and instability (Radio Random Telegraphic Signal effect, RTS) of the dark signal pattern. This is corrected by a dark signal calibration on each orbit in order to be able to remove it as accurately as possible. The operating temperature of the CCD has also been decreased to $-4{ }^{\circ} \mathrm{C}$ in order to minimize the impact of the dark signal, but unfortunately this temperature is slowly increasing, because of the evolution of thermal properties of insulating materials.

\subsubsection{Photometers description}

Two fast photometers, operating at $1 \mathrm{kHz}$, collect the star flux in the blue and the red band. Their bandwidths are $\sim 50 \mathrm{~nm}$. They share the same pupil as the IR spectrometer $\left(175 \mathrm{~cm}^{2}\right)$. The flux is split via a dichroïc that provides one spot on each detector. The detectors are a CCD matrix of $14 \times 14$ pixels of $23 \mu \mathrm{m}$. The nominal mode in occultation integrates the flux collected by a programmable area (nominally 14 lines $\times 5$ columns, corresponding to a FOV of $0.239 \times 0.671 \mathrm{~m} \mathrm{rad}$ with a focal length of $0.48 \mathrm{~m}$ ). Another calibration mode allows retrieving the individual pixel data.

The radiometric performance of the CCD in terms of noise and quantum efficiency allows it to achieve signal-to noiseratios of the order of 6 for stars of magnitude 3 during the $1 \mathrm{~ms}$ integration time.

\subsubsection{Special modes of operations}

Some secondary modes of observations and CCD reading may be programmed, mainly for calibration purposes. In the imaging mode, 33 lines of the CCD are read every $6 \mathrm{~s}$. These 33 lines cover the three useful bands: the Central band in which the star signal is usually cumulated, and the Upper and Lower bands that contain the limb brightness (if any). It allows checking to make sure that the star spectrum is well placed within the selected bands. A linearity mode allows 
Table 1. GOMOS instrument parameters.

\begin{tabular}{|c|c|c|c|c|}
\hline $\begin{array}{l}\text { Optical } \\
\text { performance parameters }\end{array}$ & Channel name & Spectral range & $\begin{array}{l}\text { Spectral sampling } \\
\text { per pixel }\end{array}$ & Spectral resolution \\
\hline Spectrometer A $2 \mathrm{hz}$ & UV(A1)-Vis (A2) & $248-690.5 \mathrm{~nm}$ & $0.31 \mathrm{~nm}$ & $0.8 \mathrm{~nm}$ \\
\hline Spectrometer B $2 \mathrm{hz}$ & IR1 or B1 $\left(\mathrm{O}_{2}\right)$ & $755-774.5 \mathrm{~nm}$ & $0.047 \mathrm{~nm}$ & $0.13 \mathrm{~nm}$ \\
\hline Spectrometer B $2 \mathrm{hz}$ & IR2 or B2 $\left(\mathrm{H}_{2} \mathrm{O}\right)$ & $926-954 \mathrm{~nm}$ & $0.056 \mathrm{~nm}$ & $0.13 \mathrm{~nm}$ \\
\hline Photometer 1 (1khZ) & Phot 1 (blue) & $473-527 \mathrm{~nm}$ & Broad band & NA \\
\hline Photometer 2 (1 khZ) & Phot 2 (red) & $646-698 \mathrm{~nm}$ & Broad band & NA \\
\hline Altitude range & $\begin{array}{l}\text { Minimum: } 5-18 \mathrm{~km} \\
\text { (night), } 15-25 \mathrm{~km} \text { (day) } \\
\text { Maximum } 250 \mathrm{~km}\end{array}$ & & & \\
\hline $\begin{array}{l}\text { Vertical } \\
\text { Resolution of } \\
\text { spectrometers }\end{array}$ & $\begin{array}{l}1-1.7 \mathrm{~km}(0.5 \mathrm{~s} \text { sample); } \\
0.2 \mathrm{~km} \text { at low altitude } \\
\text { (refraction) }\end{array}$ & & & \\
\hline
\end{tabular}

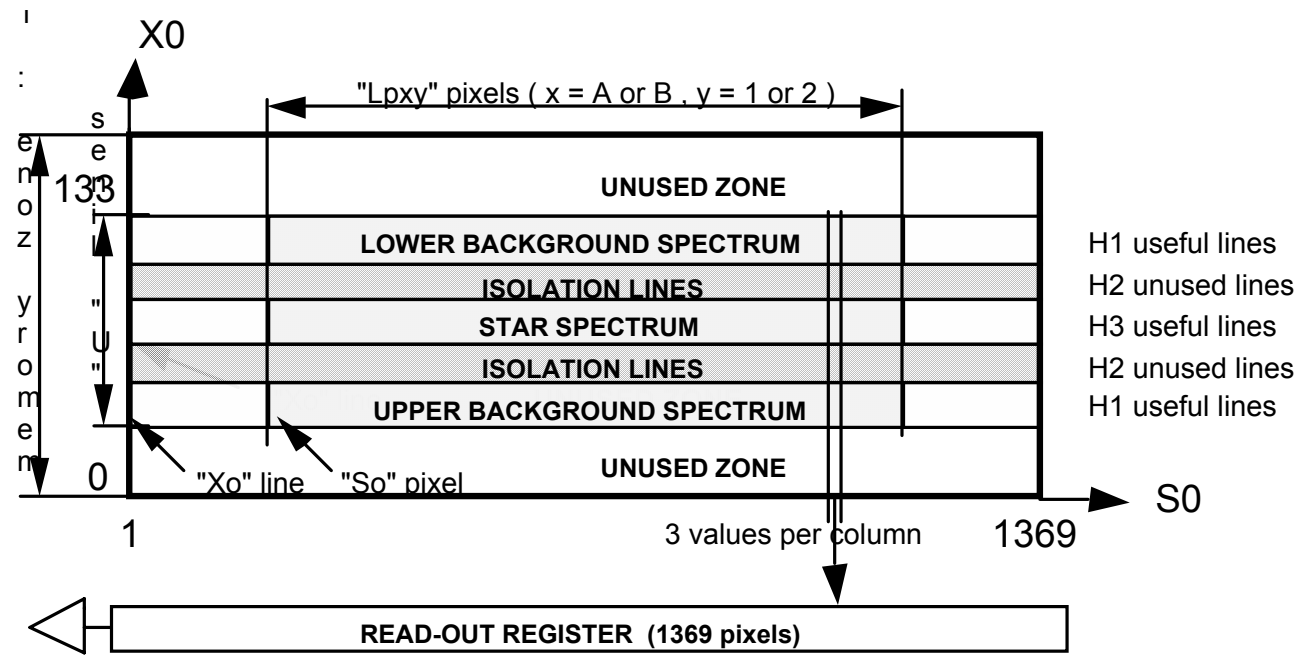

Fig. 6. Spectrometer's CCD reading for occultation mode. During the nominal mission of GOMOS, the interband region was fixed at $\mathrm{H} 1=3$ CCD lines, and the three bands are with $\mathrm{H} 1=\mathrm{H} 3=7$ lines.

measuring the same star with variable integration time from $0.5 \mathrm{~s}$ up to $10 \mathrm{~s}$, in order to determine the non-linearity of the output signal (a very minor effect). A calibration campaign called CALEX using these two modes is activated every month and the results are automatically fed to a calibration data base, to be used as an input of the pipeline processor. A dummy occultation may be made, in which the instrument is pointed to a particular celestial direction void of star, and the tracking system deactivated. In such a mode, the line of sight crosses the limb. It is used, either to record the DC pattern of all CCDs (night side), or to record the limb brightness' vertical distribution on the day-side, without any star contamination.

\subsection{Operations and earth coverage}

Owing to the combination of altitude (mean value: $799.8 \mathrm{~km}$ ), and inclination $\left(98.55^{\circ}\right)$, the precessing orbit is maintained at a constant local time with the descending node being at 10:00 LT $\pm 5 \mathrm{~min}$. The latitude of the occultation of a given star varies during the year, but repeats identically each year. The occultation latitude is represented in Fig. 7 as a function of date of year for the 70 brightest stars (up to visual magnitude $m v=2.25$ ) that can be occulted from ENVISAT on the night side. Of course, not all of them may be observed in each orbit. For some stars, the latitude varies slowly, while for others (for example, the brightest star Sirius, S001 in the GOMOS numbering convention), there is a large range of sampled latitude over the seven months during which this star can be observed with GOMOS on the night side. 


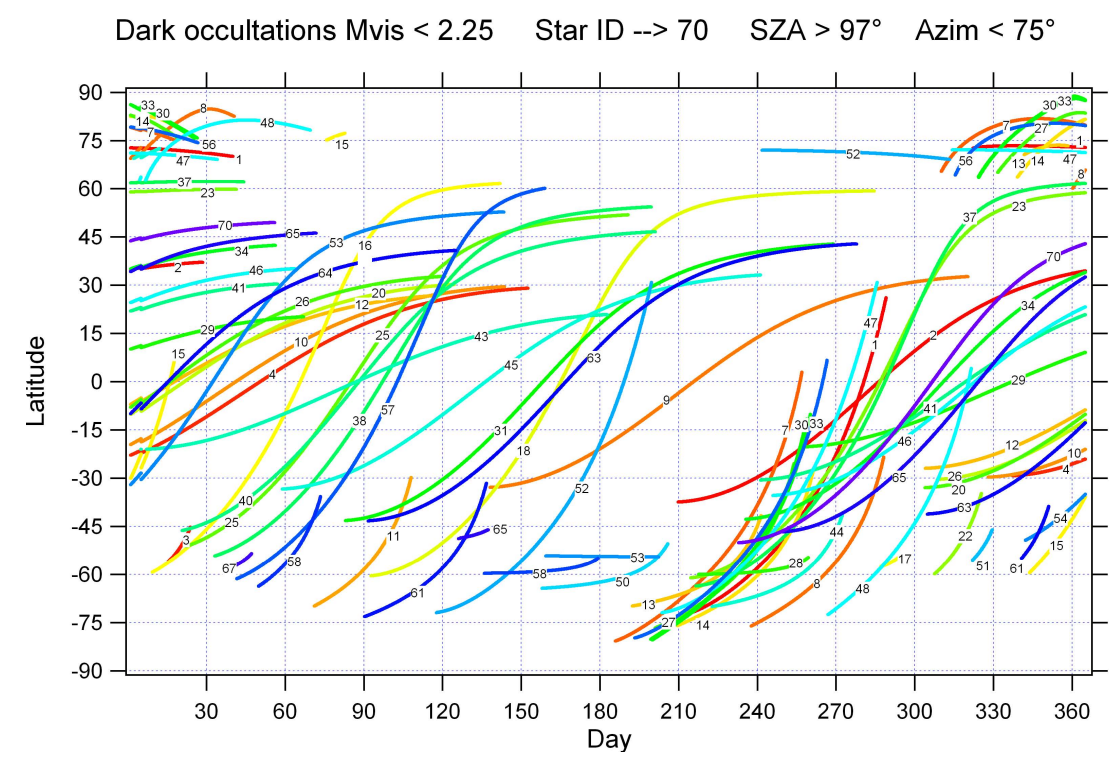

Fig. 7. Each coloured curve represents the occultation point latitude as a function of the day of the year (DOY) from the ENVISAT orbit. The number is the GOMOS star identification number, ordered by increasing visual magnitude. Only occultations in dark limb conditions, and with an azimuth angle $<75^{\circ}$ are represented. Scintillations have a greater effect on transmission spectra for larger azimuth angles. The pattern repeats identically every year.

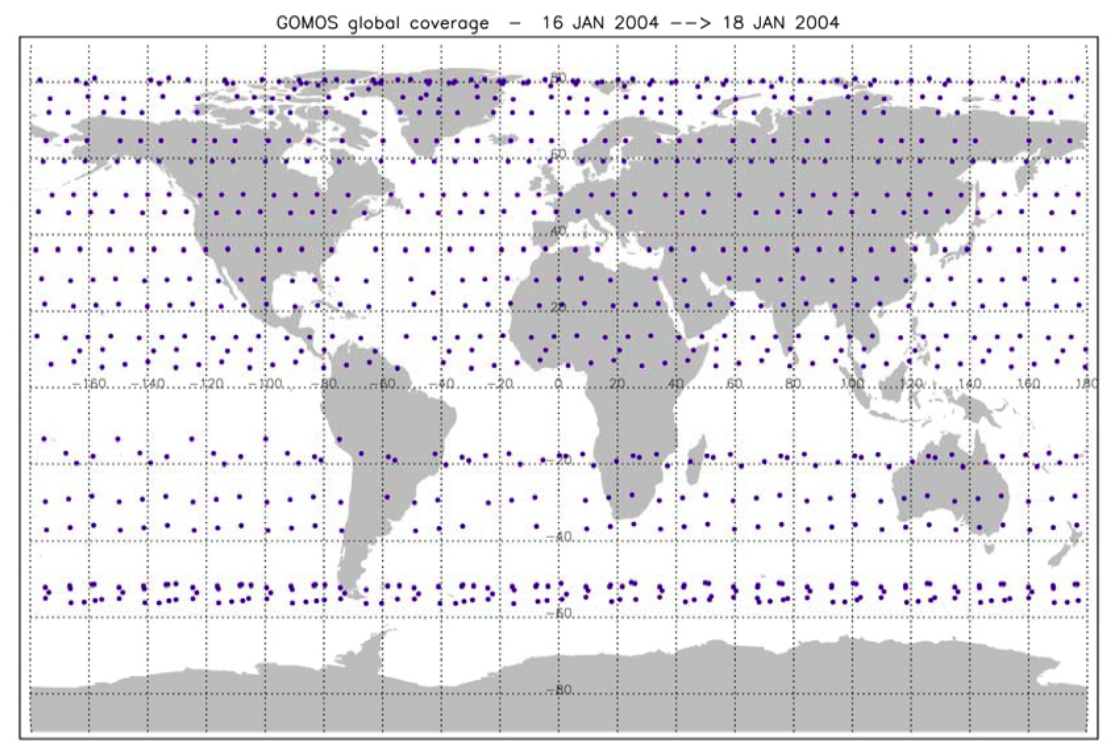

Fig. 8. Each point represents the location of one occultation, performed during a period of 3 days (16-18 January 2004). The pattern is regular in longitude, but irregular in latitude, since it depends on the availability of stars. Because of the geometry of occultation, one measurement is a horizontal average over a few hundred $\mathrm{km}$, which adds somewhat to the coverage.

Still, from one orbit to the next, the occultation latitude does not change much. Therefore, the same star may be observed at each orbit for some weeks. The orbital period of ENVISAT at $100.6 \mathrm{~min}$ is maintained with $14+11 / 35$ orbits per day; therefore, after 3 days, the occultation point of a given star is almost at the same latitude and longitude, with 43 points uniformly spread in longitude along a circle of constant latitude. As noted earlier, the decorrelation time of ozone at a given point in the stratosphere is of the order of 3 days.

Along the polar orbit of ENVISAT the latitude is changing rapidly. Once an observed star is fully occulted, GOMOS is rallying towards another star, which will be occulted at a different latitude. The latitude coverage depends on the 


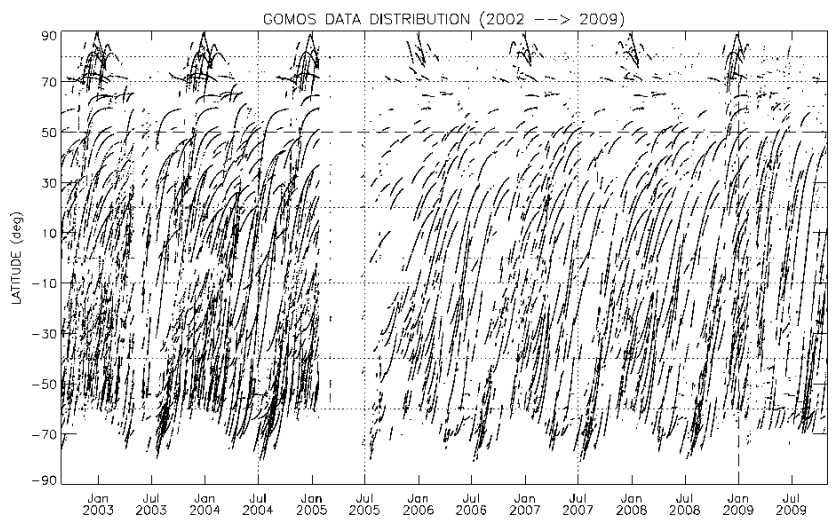

Fig. 9. Latitude distribution of all night time occultations performed by GOMOS (2002-2009). One arc represents many successive occultations of the same star, moving in latitude from one orbit to the next. The coverage was somewhat reduced after the 2005 malfunction of the Steering Mirror Mechanism. Polar latitudes are reached only during winter time.

availability of bright stars, and is not regular. Figure 8 indicates a typical coverage of GOMOS occultations for a period of 3 days, while Fig. 9 indicates the coverage over 7 years of GOMOS night-time occultations. The pattern repeats itself from one year to the next.

In Fig. 9 a continuous arc represents many occultations of the same star on successive orbits, covering all longitudes and slowly varying in latitude. Essentially, the Earth is rotating rapidly inside the orbit plane/star system, while the orbit plane rotates by $1^{\circ}$ per day w.r.t. the star system.

There are some interruptions in time that were caused by some malfunctions of the Steering Front Mirror assembly mechanisms, as described later. In particular, after the malfunction of early 2005, the coverage is less dense because the change of azimuth from one star to the next was restricted for safety reasons. The average number of occultations per day decreased from 400 to $250-300$ when operations were resumed in 2005.

The observation of a particular sequence of star occultations requires the uploading of a set of star-pointing parameters that control the initial pointing direction and the apparent angular velocity (due to the satellite motion) of individual target stars. The observing programme for GOMOS is established on the ground at ESRIN with the help of Starsel software that optimizes by picking up the brightest stars available, knowing the occultation time and duration for one star, and the time to rally to another star. At pre-launch a list of 1000 stars had been defined, named for GOMOS purposes from S001 (Sirius) to S1000, ordered by increasing magnitude (i.e., decreasing order of brightness at $550 \mathrm{~nm}$ ). Actually, the dimmest star that was useful for providing a decent ozone accuracy is $\mathrm{S} 185$, at magnitude $\mathrm{mv}=3.08$.
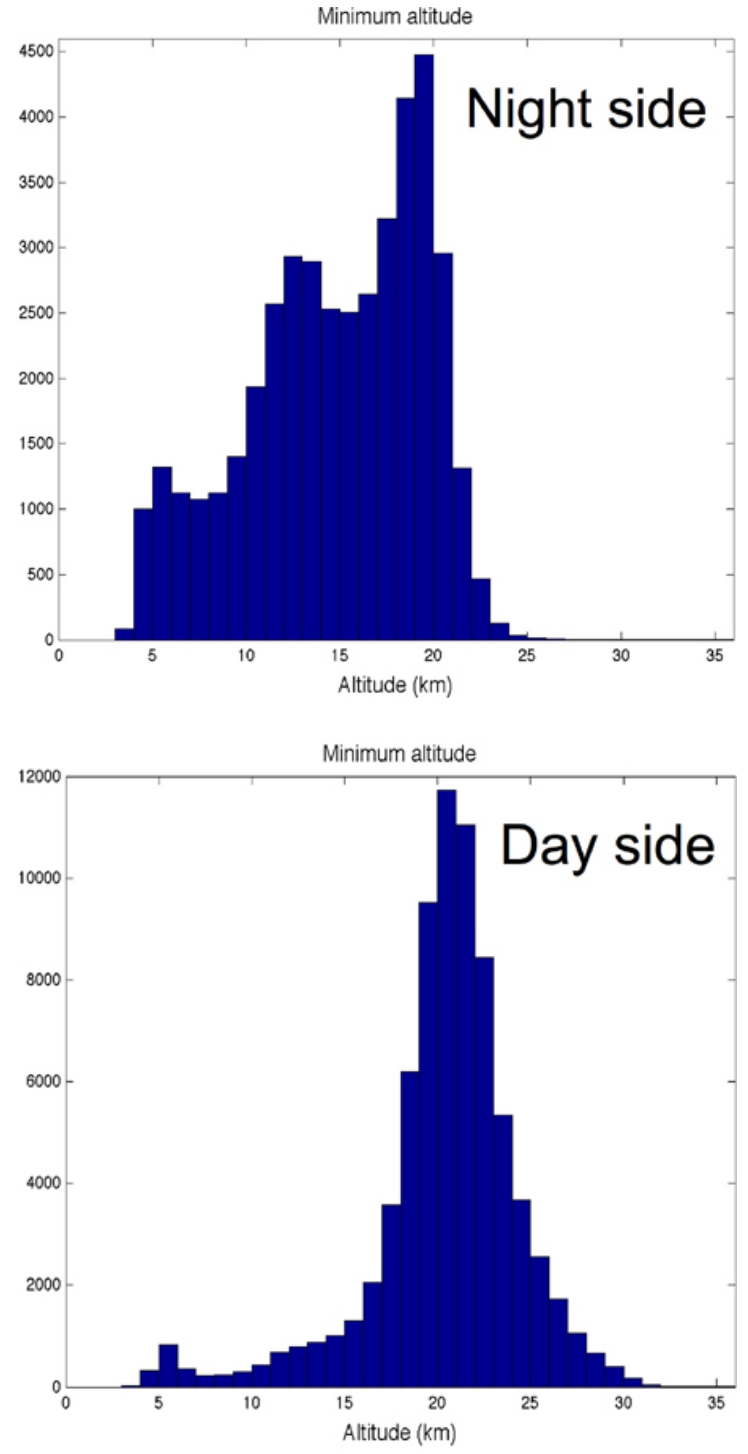

Fig. 10. The distribution of the termination altitudes during all the occultations of 2004. Top: dark limb occultations only. Bottom: bright limb occultations only. In dark limb the average is $15 \mathrm{~km}$.

Planets have also a number, along with a series of $\sim 50$ dark regions in the sky (DSA, Dark Sky Area), void of stars, which can also be occulted during a dummy occultation, to measure the Dark Charge (at high altitude) and the limb brightness or to characterize stray-light contamination.

Altitude coverage: once a star is acquired at an altitude $>120 \mathrm{~km}$, it is tracked by the SATU as long as possible by acting on the plane mirror, keeping the image of the star in the centre of the slit. It is lost either because of the presence of clouds, or due to strong scintillations, which move the star vertically and modulate strongly the signal, both ways (brightening and dimming). The star is declared "lost" when the star tracker does not find the star during more than $0.1 \mathrm{~s}$ (10 samplings). Figure 10 shows a histogram of the 
altitudes at which a star was lost during all occultations of 2004 (separately for day and night). The stars can typically be tracked from the top of the atmosphere $(120-150 \mathrm{~km})$ down to 5-20 km tangent altitude for night occultations, and $15-25 \mathrm{~km}$ on the day side. It seems that when no clouds are present, stars can be followed as low as $5 \mathrm{~km}$ altitude (especially bright stars). The presence of clouds in the field-of-view leads to losing the signal. The lowest altitude reached in occultations of bright stars can potentially provide valuable information about cloud top height (Tamminen et al., 2010). Its geographical distribution is informative. For instance, in the South Polar night measurements are often terminated at $15-18 \mathrm{~km}$, most probably because of frequent occurrence of polar stratospheric clouds. In the tropics, there is a concentration over the continents and East Pacific of high altitude clouds (cirrus and convective clouds, Tamminen et al., 2010).

\subsection{Typical results}

\subsubsection{Stellar spectra}

At each occultation, when the star is acquired and tracked by the SATU (Star Acquisition and Tracking Unit), at high altitude, (usually around $120 \mathrm{~km}$ ) the ten first spectra are averaged together to give the Reference Star Spectrum, $S_{0}$, outside the atmosphere. Figure 11 represents spectra for various stars, illustrating two features of GOMOS. The first feature is that the signal is dimmer when the star magnitude is larger (the magnitude of a star is defined according to its flux at $550 \mathrm{~nm}$ ). Of course, brighter stars are favoured in the selection of stars, but in order to get a good latitude coverage, one has to select quite often dimmer stars (up to magnitude 3), due to a lack of bright stars at a given latitude. Obviously, the accuracy of ozone (and other species) retrieval depends on the star brightness. The second feature is the fact that hot stars have much more UV flux than cool stars of equal magnitude, allowing ozone retrieval with the Hartley's absorption continuum, peaking at $255 \mathrm{~nm}$ (the shortest wavelength of GOMOS is $248 \mathrm{~nm}$ ). Each spectrum is measured for $0.5 \mathrm{~s}$, and the signal per pixel in electrons gives the indication of the shot noise, the main source of error for the spectral transmission: $1 \%$ for a signal of 10000 electrons. The pixel spectral size (sampling) is given in Table 1.

Note in Fig. 11 the strong Fraunhofer Hydrogen lines in the spectrum of star S005 (GOMOS identification number of the star Vega, the 5th brightest in the sky), allowing a good in-flight wavelength calibration of the spectrometer, and a relative lack of signal around $380 \mathrm{~nm}$, which is an instrumental effect: this is where the spectrum of the star produced by a holographic grating is divided in two parts, reaching two different CCDs (UV and Visible) with some resulting vignetting.

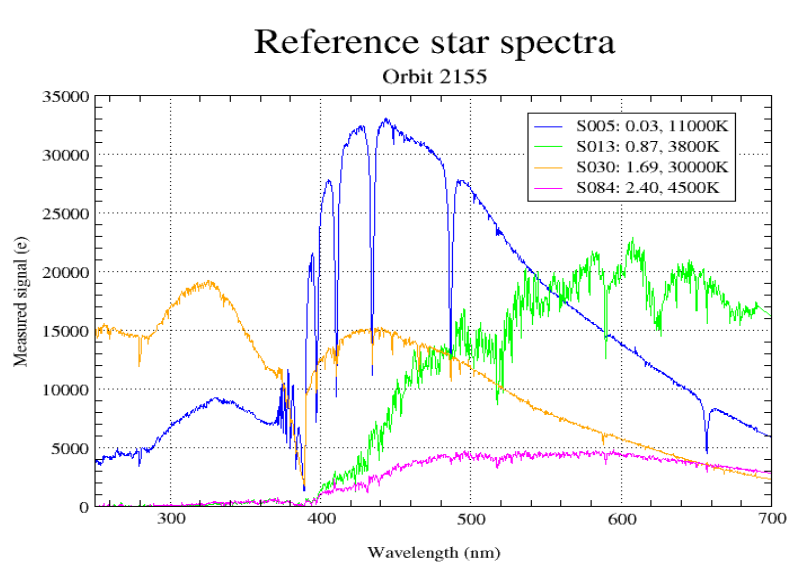

Fig. 11. Spectra of different stars observed by GOMOS. Hot stars contain more UV light than cool stars and are more suitable to the measurement of mesospheric ozone. Just below $400 \mathrm{~nm}$, the low signal is due to the separation of the spectrum on two CCDs. The GOMOS ID number of the star is indicated, with its visual magnitude and temperature.

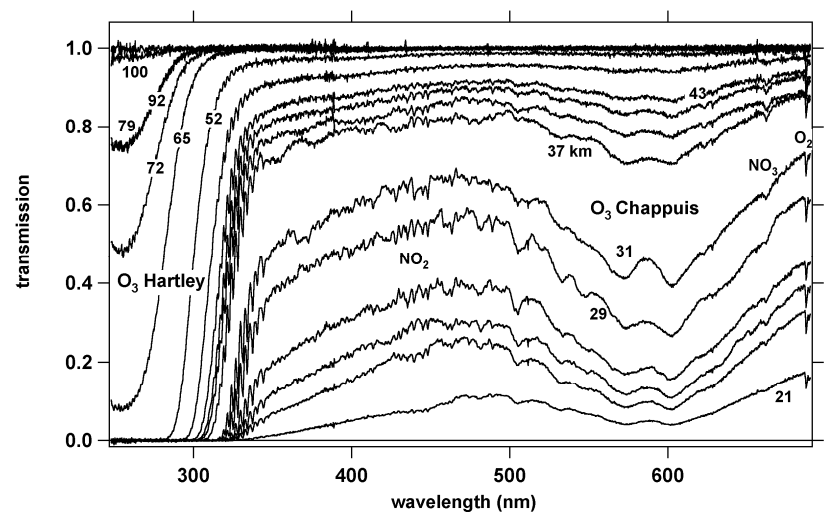

Fig. 12. Atmospheric transmission spectra along the line of sight from GOMOS to the star Sirius, for different tangent altitudes.

\subsubsection{Atmospheric transmissions}

Figure 12 shows the spectral transmissions, $T_{m}$, measured at a number of tangent altitudes, $z$, during one occultation of the star Sirius (the brightest in the sky). $T_{m}(z)$ is obtained by ratioing $S_{m}(z) / S_{0}$, where $S_{m}(z)$ is the measurement of the spectrum through the atmosphere obtained at altitude $z$, during $0.5 \mathrm{~s}$ (altitude integration over $1.7 \mathrm{~km}$ at most, depending of the distance of the star from the orbital plane). In this particular case, the minimum altitude was less than $10 \mathrm{~km}$, while for dimmer stars, the tracker may lose the star between 20 and $10 \mathrm{~km}$. From UV to $700 \mathrm{~nm}$, one can recognize on the transmission spectra:

- the strong Hartley's absorption continuum at 250$280 \mathrm{~nm}$, allowing measurement of ozone up to $100 \mathrm{~km}$, 


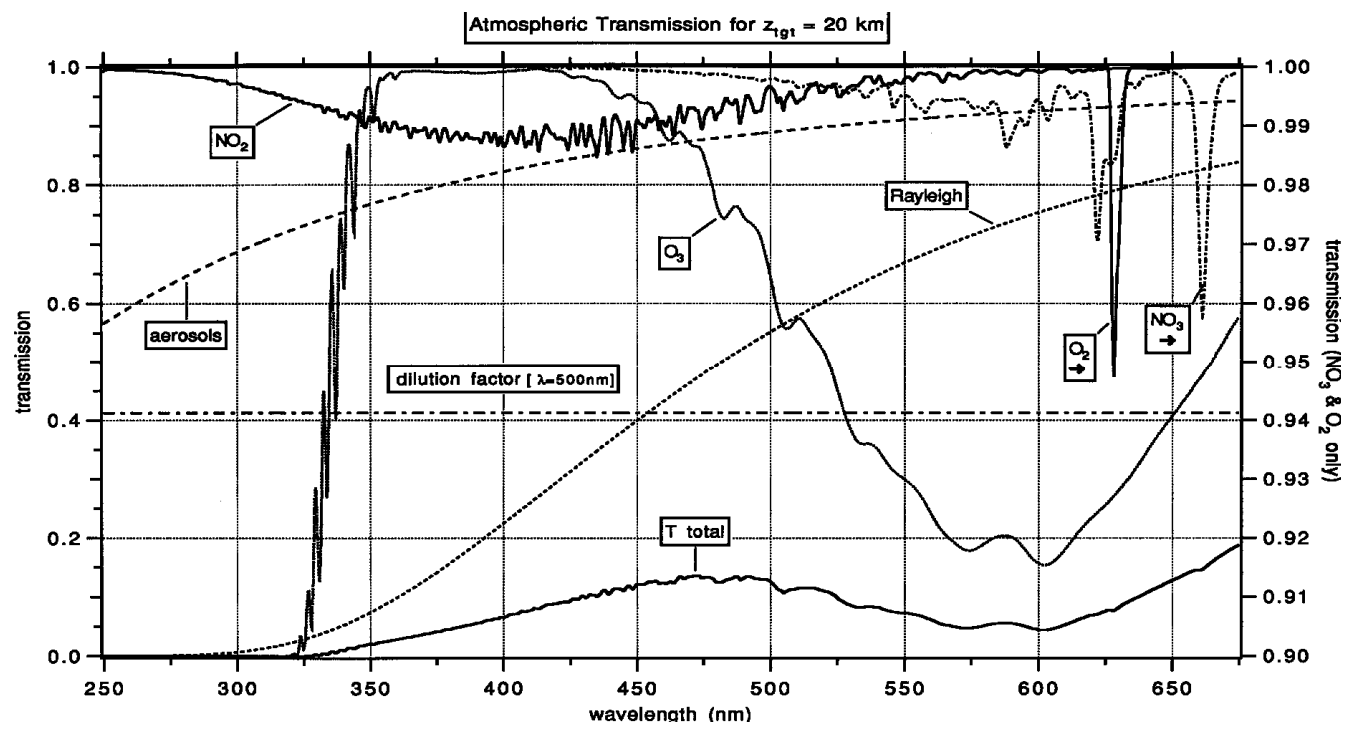

Fig. 13. Forward simulation of the atmospheric transmission. The contribution of various absorbers at a tangent altitude of $20 \mathrm{~km}$ are indicated as well as the total transmission. The dilution factor is due to atmospheric refraction, and is about 0.4 at $20 \mathrm{~km}$ altitude.

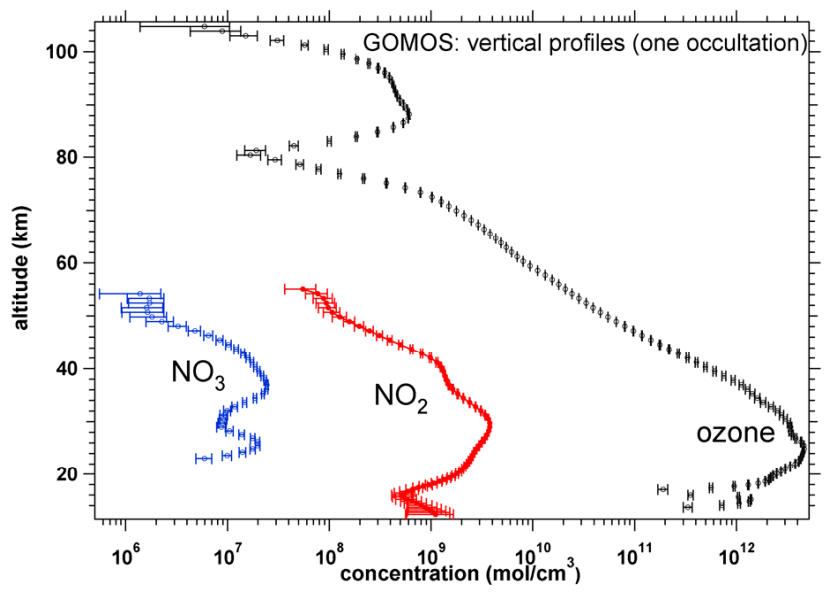

Fig. 14. Vertical profiles and their error bars for ozone, $\mathrm{NO}_{2}$ and $\mathrm{NO}_{3}$ retrieved from one single occultation of the star Sirius.

- the Huggins bands of ozone at 300-340 nm (visible from 29 to $52 \mathrm{~km}$ ),

- small spectral features around $440 \mathrm{~nm}$ due to $\mathrm{NO}_{2}$,

- the broad absorption Chappuis band of ozone, from 480 to $700 \mathrm{~nm}$, with pronounced spectral features,

- a small feature at $656 \mathrm{~nm}$ due to $\mathrm{NO}_{3}$,

- a small $\mathrm{O}_{2}$ band at $670 \mathrm{~nm}$,

- the Rayleigh extinction of air, giving the slope around $400 \mathrm{~nm}$ (visible essentially below $30 \mathrm{~km}$ ).

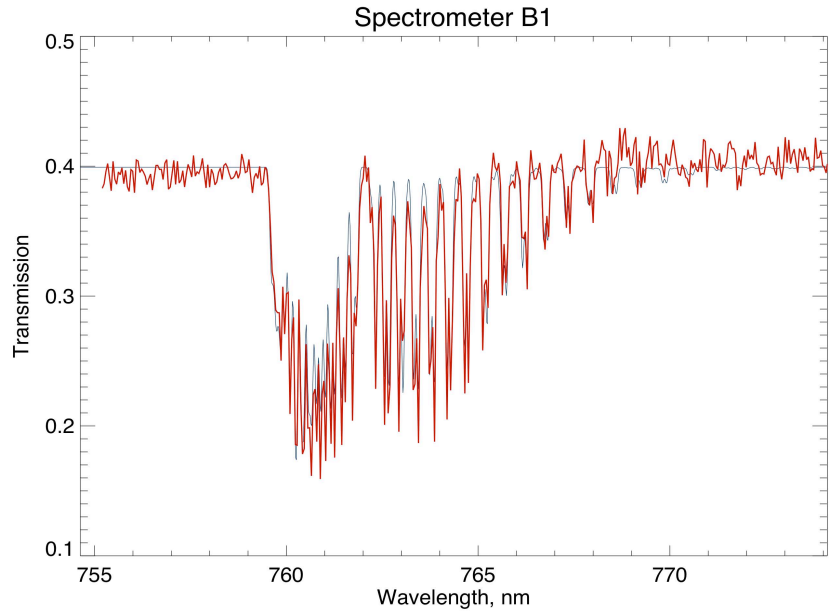

Fig. 15. Spectral transmission measured in the $\mathrm{O}_{2}$ band (called "A band", around $760 \mathrm{~nm}$ ), compared with a HITRAN model (adjusted to the baseline). The level of $T_{\bmod }$ was adjusted to the level of the measured transmission, which is well below 1 , mainly because of refraction effects.

There are also some irregularities (conspicuous on the $37 \mathrm{~km}$ transmission around $400 \mathrm{~nm}$ ), which are due to imperfect corrections of the scintillation effects, as is explained further below.

Figure 14 displays the vertical profile of ozone, $\mathrm{NO}_{2}$ and $\mathrm{NO}_{3}$ retrieved from one occultation of the star Sirius. For ozone the retrieval extends from 16 to $106 \mathrm{~km}$ (lower stratosphere to mesosphere), with a range of concentration covering six orders of magnitude. This exceptional dynamic range is possible because of a corresponding large range of absorption cross section over the GOMOS wavelength 


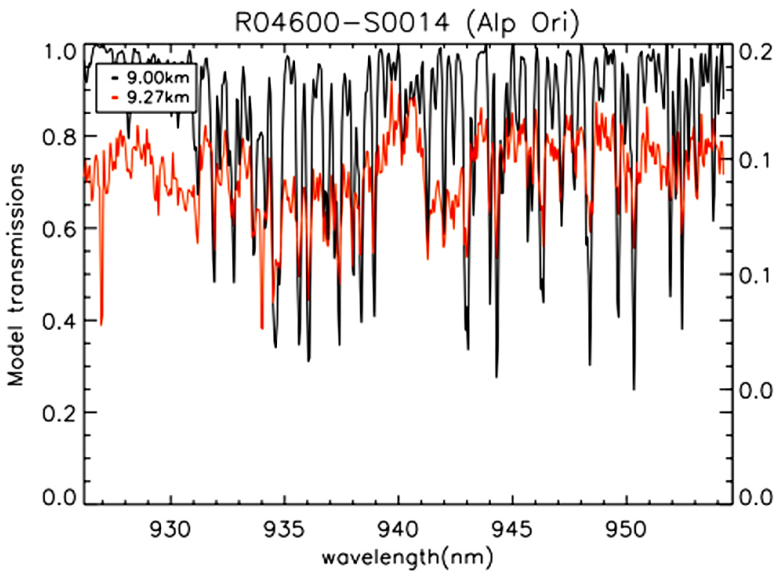

Fig. 16. GOMOS transmission obtained with the star Alpha Orionis (S0014 is the GOMOS ID number) at a tangent altitude of $9.27 \mathrm{~km}$ just below the tropopause (red curve, right scale), and the model transmission at $9 \mathrm{~km}$ (black curve, left scale). There are features in the measured spectra that do not come from $\mathrm{H}_{2} \mathrm{O}$ absorption, but rather from an imperfect correction of the severe pixel-to-pixelnon-uniformity affecting the $\mathrm{CCD}$ of spectrometer $\mathrm{B} 2$ in the range $940 \mathrm{~nm}$. This was taken on ENVISAT orbit $\mathrm{n}^{\circ} 4600$.

domain. GOMOS is the first instrument providing systematic $\mathrm{NO}_{3}$ profiles. This species exists only at night, being rapidly photo-dissociated by sunlight.

Figures 15 and 16 show the spectral transmission obtained respectively in the Oxygen $\mathrm{O}_{2}$ band (called the A band) around $760 \mathrm{~nm}$, and in the water vapour band around $936 \mathrm{~nm}$. Measured transmission spectra are compared with a simulation of the model transmission $T_{\text {mod }}$. It is obtained with HITRAN line-by-line calculations at the highest possible spectral resolution, then convoluted to the actual resolution of the instrument, considering only these gases. The high degree of resemblance should be noted between the data and the model for $\mathrm{O}_{2}$, demonstrating the high spectral quality of the data, considering the weakness of the stellar source. For $\mathrm{H}_{2} \mathrm{O}$, all absorption features predicted by the model are present in the observed spectra. However, there are also other features in the measured spectra that do not come from $\mathrm{H}_{2} \mathrm{O}$ absorption, but rather from an imperfect correction of the severe pixel-to-pixel nonuniformity affecting the $\mathrm{CCD}$ of spectrometer $\mathrm{B} 2$ in the range $940 \mathrm{~nm}$.

\subsubsection{Scintillations observed from fast photometers}

Scintillations are one aspect of refraction. They are due to small irregularities in the air density vertical profile (and therefore, temperature). Figure 17 shows an example of scintillation measurements by the GOMOS fast photometers. The rms variation of relative fluctuations of intensity recorded by the photometer rapidly grows with decreasing altitude (roughly proportional to air density) until it saturates at values $\sim 1$ at an altitude of $\sim 30 \mathrm{~km}$. The spectrometers

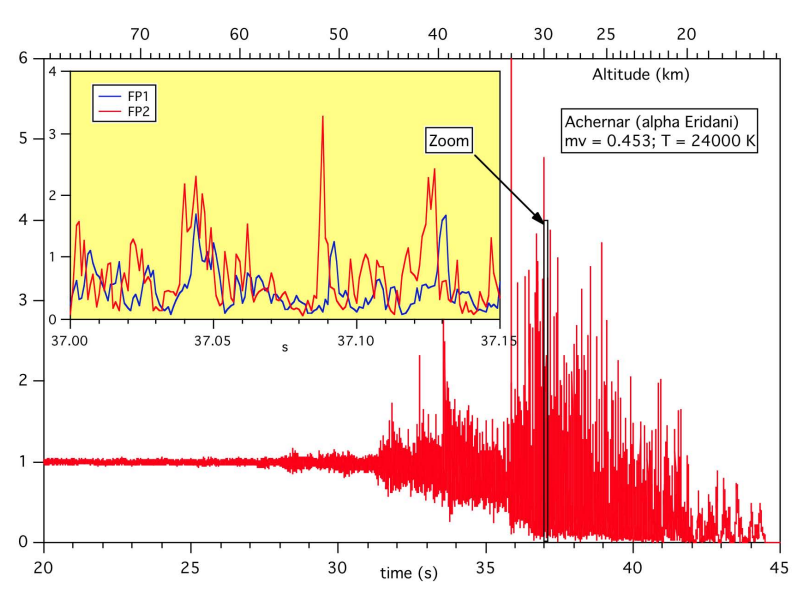

Fig. 17. Example of real scintillation recorded at $1 \mathrm{kHz}$ by Gomos. Tangent altitude is shown on a non-linear scale at the top of the graph. The zoom shows the signals from "blue" and "red" photometers (see the text for details).

have a much lower sampling rate of $2 \mathrm{~Hz}$. However, the photometer signal averaged down to $2 \mathrm{~Hz}$ still exhibits fluctuations (not shown here). The amplitude of these fluctuations range from $\sim 4 \%$ up to $\sim 20 \%$ at altitudes below $30 \mathrm{~km}$. This modulation of the transmittances caused by scintillation significantly exceeds the instrumental noise, especially in case of very bright stars. Therefore, the signals from the two photometers are used to correct the deformations of transmission spectra as much as possible before the spectral inversion.

In addition, the photometers' data may be exploited to characterize the turbulent state of the atmosphere (Sofieva et al., 2007a, b, 2009a; Gurvich et al., 2007), and derive a vertical profile of the temperature with high resolution, from the measured time delay between the blue peaks and the red peaks, which are produced by the same air temperature irregularity: the blue rays are more refracted than the red rays, and are therefore encountered later by GOMOS during the occultation (insert of Fig. 17).

\section{Problems encountered during the GOMOS mission}

During the 8 first years of the GOMOS mission (20022009), some difficulties and problems were encountered that deserve to be described in some detail, as well as related mitigation schemes. These include: an unexpected increase of the Dark Charge (DC) of the CCD detectors, the scintillations produced by the isotropic component of temperature fluctuations, some malfunctions of the orientation mechanisms, a component of stray light on the day side, and the "cosmic hits" produced by energetic particles encountered when ENVISAT is in the South Atlantic Anomaly (SAA). 


\subsection{Dark charge of the CCDs}

The CCDs used as detectors in the GOMOS spectrometers were procured from EEV, Ltd. They are back-side illuminated, ensuring a good quantum efficiency for the whole wavelength range of the spectrometers. They were advertised as having a very low Dark Charge (DC), using the MMPP technology, which should have precluded the usual practice of cooling the CCDs to $-40^{\circ} \mathrm{C}$. The thermal concept of the instrument was therefore simplified, and a good thermo-mechanic stability was ensured (especially important for avoiding spectral shifts).

Unfortunately, some pixels soon showed a different behavior, which affected all of them after 2-3 years in orbit. The DC increased by more than a factor of 10 , and also showed variations of mean level much greater than expected from Poisson statistics, on scale times of tens of minutes. This phenomenon is referred as RTS in the literature (RadioRandom Telegraph Signal), caused by radiation belt energetic particles changing the structure of the silicon in the pixel. Instead of being uniform along the CCD, the DC presented a highly structured pattern: the DCNU (Dark Charge Non-Uniformity).

The situation was mitigated by devoting at each orbit one full dummy occultation, pointing at a Dark Sky area and recording the exact pattern of the DCNU. This occultation occurs near the ascending node (crossing the equator), in the depth of the night-side to avoid any light contamination. This recorded pattern is then used to correct by subtraction all the occultations of the following orbit.

Nevertheless, the mean DC increases as a function of time, as shown on Fig. 18 for a combination of two causes: the increase of temperature of the instrument, because of the degradation of the thermo-optical properties of the insulation layers, and the increase of the RTS. It is possible to disentangle the two effects, since the law of DC change with temperature is well known. Figure 19 shows the histograms of the number of pixels showing a certain dark charge in electrons per $0.5 \mathrm{~s}$, separately for the various years of operation. In 2003, some pixels were still showing low dark charges, while larger and larger values are seen in the following years. It clearly affects the accuracy of the various gases' retrieval, mainly for the dimmest stars, whose signal is competing with the dark charge, as shown in Keckhut et al. (2010) by comparison with microwave- and lidar groundbased instruments.

\subsection{Problems with scintillations}

Scintillations are the result of interaction of light waves with air density irregularities (Gurvich and Brekhovskikh, 2001; Dalaudier et al., 2001). It is known that there are two types of air density irregularities in the Earth stratosphere: anisotropic irregularities, which are stretched along the Earth surface (generated mainly by internal gravity waves), and

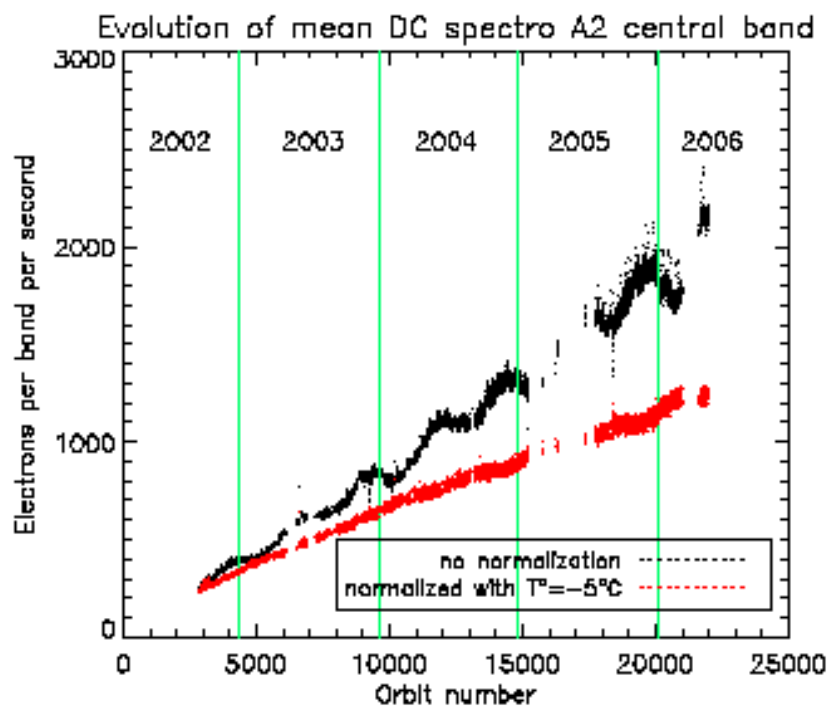

Fig. 18. Mean Dark Charge for spectrometer A2. The black curve shows seasonal variations due to the position of the sun and thus to the change of the temperature of the CCD. The red curve has been obtained by normalizing the black curve at a reference temperature of $-5^{\circ} \mathrm{C}$. Therefore, the red curve shows that an increase of Dark Charge independent of the temperature still exists, due to radiation damage.

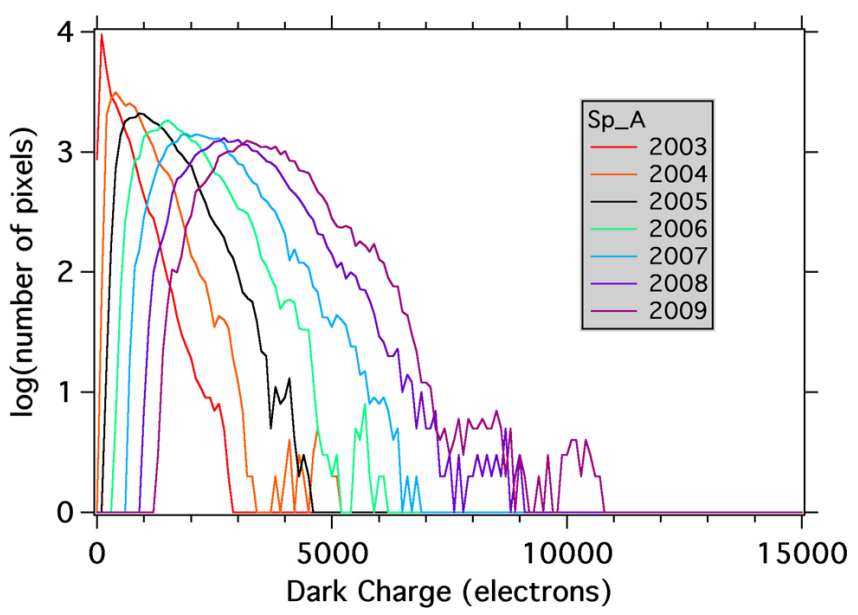

Fig. 19. Histograms of Dark Charge of Spectrometer A CCD detectors. The bins are 100 electrons; the vertical scale is the Log 10 of the number of pixels having the specific dark charge of the bin. They are collected around the 1st of January, during 20 Dark Sky Area observations dedicated to the measurement of Dark Charge. The Dark Charge is increasing from year to year, due to accumulated radiation damage.

isotropic irregularities (turbulence), which appear as a result of gravity wave breaking and due to different instabilities. These irregularities produce scintillations at the observation site. 
Before the launch of ENVISAT, the isotropic component was thought to be small in the stratosphere. However, the analysis of the fast photometers, and of the wavelength transmissions measured by the spectrometers showed that it was more important than expected. As a result, the scheme that was designed to correct the spectrometer transmission did not achieve a complete correction.

When a star lies in the orbital plane $\left(\mathrm{Az}=0^{\circ}\right)$, GOMOS will observe exactly the same density vertical structure at all wavelengths (but various times); in such a case, the scintillation correction will be perfect, even in the presence of isotropic fluctuation. For a star which is not in the vertical plane (the usual situation), the occultation point will have an oblique trajectory at the limb (Fig. 50). If the fluctuations are purely anisotropic (the vertical structure is conserved horizontally), the scintillation pattern will be exactly the same as if the occultation were in the vertical plane. It was found that this is not the case: there is always an isotropic component to the fluctuations, which results in an imperfect correction of the transmission spectra, becoming worse as the obliquity increases.

The impact on the accuracy of ozone retrieval of such an effect has been studied (Sofieva et al., 2009). The effect is significant between 20 and $40 \mathrm{~km}$, increasing the error on ozone local density from $<1 \%$ up to $2 \%$ for a bright star, and up to $4-8 \%$ for dim and cool stars of magnitude $>2.5$, $T<6000 \mathrm{~K}$ (Tamminen et al., 2010).

\subsection{Stray light}

The internal straylight is the light contained in the FOV (Field Of View) limited by the slit, but whose photons are mainly light scattered by the grating and it is most manifest as light from the star collected in the background bands (up to $1.5 \%$ of the light collected in the Star Central band in the UV). At present time, no correction of the internal stray light is implemented.

External stray light is light that makes its way through the slit, although its origin is outside the nominal FOV defined by the slit at the focal plane of the telescope. There are mainly two sources of external stray light. One is the solar light scattered by some ENVISAT hardware into the baffle and optics of GOMOS. The other comes from the sunilluminated limb or the nadir. It bears a strong tropospheric signature of $\mathrm{H}_{2} \mathrm{O}$ and $\mathrm{O}_{2}$ absorption. The external stray light of solar origin does not go through the ozone layer and therefore contains some UV even at maximum ozone absorption wavelengths $(255 \mathrm{~nm})$. At the present time, no correction of the external stray light is implemented, because most of it is the same in the three bands, and is subtracted at the same time as the bright limb contribution (see below). Still, geometrical computations are performed to characterize the illumination conditions of GOMOS, ENVISAT and the tangent point of occultation at the limb at a reference altitude (the nominal value is $50 \mathrm{~km}$ ). The illumination condition is
Percentoge of cosmic ray hits per profile
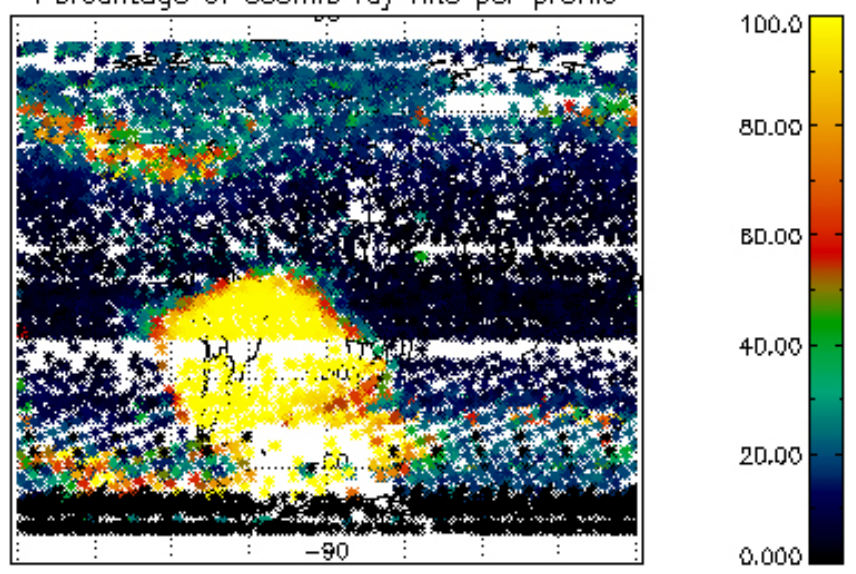

Fig. 20. Each point of the world map is the position of ENVISAT at the time of one occultation recorded by GOMOS during the month of November 2004. The color coding describes the percentage of $0.5 \mathrm{~s}$ samples of CCD spectrometers that have at least one cosmic hit. Both North and South auroral zones are visible, but the region most affected is the South Atlantic Anomaly.

given in the flag stored in the Level $1 \mathrm{~b}$ product (PCD ILUM). This index may be also used as a guide for the stray light contamination.

\subsection{Cosmic hits}

They are generated by high energy particles trapped in the radiation belts, and manifest themselves by a much larger content of electrons in a pixel, or a few adjacent pixels (depending on the angle of the trajectory to the plane of the CCD). They are tracked and flagged by the pipeline software of Level $1 \mathrm{~b}$. They affect the measurements mainly when ENVISAT is in the South Atlantic Anomaly, or at high latitudes in the auroral zones, as can be seen on Fig. 20. Only the flagged pixels are ignored during the retrieval of constituents, and the occultation as a whole is still valid.

\subsection{Orientation mechanism malfunctions}

Figure 9 shows some interruptions of the data, all of which were caused by some malfunction of the Steering Front Mechanism (SFM). In May and July 2003, a malfunction of the "voice coil" commanding the azimuth motion forced ESA to switch GOMOS to a redundant system, Side B. In 2005, a similar problem affected the elevation mechanism, which could not track the star completely. It was found through thorough investigation that the problem occurred mainly when a large excursion in azimuth was scheduled between two consecutive stars. Therefore, GOMOS was then operated in a limited range of azimuth, a window of $30^{\circ}$, which limits were optimized by one of us (Hauchecorne) in 
order to still get a good sky coverage. It is obvious from Fig. 9 that the overall sampling is coarser after this second failure, and GOMOS is still operated in this mode at the time being (December 2009).

\section{Retrieval algorithms}

\subsection{Overview}

In this section we describe briefly the algorithms used to retrieve vertical profiles of atmospheric parameters from GOMOS raw measurements (Level 0 data). A more complete description may be found in Kyrölä et al. (2010a) as a part of this GOMOS ACP special issue.

The species covered by GOMOS are $\mathrm{O}_{3}, \mathrm{NO}_{2}, \mathrm{NO}_{3}, \mathrm{H}_{2} \mathrm{O}$, $\mathrm{O}_{2}$, as well as aerosols and temperature: which together constitute the so-called Level 2 GOMOS data products. The Level 2 product contains also the line densities of measured species, and the ECMWF data for air (both local density and temperature, and integrated line density). The measurements cover altitudes of $5-150 \mathrm{~km}$. The vertical range of valid data is somewhat variable, in particular with the star type and brightness: for ozone, $10-100 \mathrm{~km}$; for $\mathrm{NO}_{2}$ and $\mathrm{NO}_{3}, 20-$ $50 \mathrm{~km}$; aerosols, $15-40 \mathrm{~km}$. In special occurrences, $\mathrm{NO}_{2}$ is seen up to $70 \mathrm{~km}$.

In a very natural way, the data processing of GOMOS includes the extraction of the atmospheric transmission as the intermediate product, defining the output of the processing that proceeds from raw measurements (Level 0) to Level $1 \mathrm{~b}$ (atmospheric transmission). Quite obviously also, the data flow of GOMOS is divided into individual data sets each covering one occultation, since each of them can be analyzed independently from the others. The data processing is somewhat different, according to the illumination conditions at the tangent point as defined earlier.

The algorithms discussed here are used in the Envisat GOMOS ground segment supported by ESA. The algorithms have been developed under a contract between ESA and the company ACRI France in Sophia-Antipolis, France. The scientific institutes, the Finnish Meteorological Institute, the Service d'Aéronomie (now LATMOS) and the Institut d'Aéronomie Spatiale de Belgique, have been the scientific partners of ACRI, forming together the GOMOS Expert Support Laboratory coalition (ESL) in charge of the algorithm development. The GOMOS Level $1 \mathrm{~b}$ and Level 2 algorithms are described in great detail in the GOMOS Detailed Processing Model document (GOMOS ESL, 1998).

\subsection{Level 0 to Level 1b processing overview and Level 1b data products}

The data processing to reach Level $1 \mathrm{~b}$ spectrometers' products consists basically of:

- correction of instrumental effects,
- separation of stellar light from limb background light and calculation of total transmission,

- geolocation of each measurement.

The data processing requires good orbit knowledge and good knowledge of instrument characteristics (dark charge, wavelength calibration, wavelength resolution, etc.), based on ground calibration and in-flight additional measurements (with special monitoring modes of GOMOS). In addition, an atmospheric vertical profile is needed, in order to account for atmospheric refraction of the ray path for a more precise geolocation of the transmission measurement.

The Level $1 \mathrm{~b}$ data processing is identical for both spectrometers A (UV-Vis) and $\mathrm{B}$ (for $\mathrm{O}_{2} 760$ and $\mathrm{H}_{2} \mathrm{O}$ $936 \mathrm{~nm})$.

\section{Level 1b data products}

The Level $1 \mathrm{~b}$ data products are listed below, with their sampling rate. They are grouped occultation-by-occultation (about $\sim 30$ per orbit). Some comments on these data products are listed below, while details on the data processing are given later.

Reference star spectrum (once per occultation): it is obtained by averaging the 10 first spectra obtained above $120 \mathrm{~km}$ during the occultation to minimize the noise.

Reference atmospheric profile $(2 \mathrm{~Hz})$ : this density profile is extracted from a meteorological field analysis (ECMWF) combined with a climatological model at higher altitudes (MSISE-90, Hedin, 1991). It is used during Level 1b processing to compute the refraction of the LOS, by full raytracing computation. It is also used in Level 2 processing to fix air density (Rayleigh extinction) and is contained in the Level 2 product.

Full transmission spectra and covariance $(2 \mathrm{~Hz})$ : this spectrum is obtained by dividing each spectrum in one occultation by the reference star spectrum. It is called "full" because it is the actually measured transmission, not corrected for refraction effects (dilution, scintillation, chromatic refraction) nor for variable PSF (Point Spread Function). The covariance (here, in fact, the variance of each pixel signal) is computed from analysis of the $S / N$ ratio.

Central Background estimate $(2 \mathrm{~Hz})$ : this is the estimated background contribution to the total signal in the central band, which is subtracted to yield the pure stellar signal.

Photometers data $(1 \mathrm{kHz})$ : correction to raw data includes the unfolding of the data (when they suffer from an overflow) and the subtraction of Dark Charge.

SATU data $(100 \mathrm{~Hz})$ : this is the position of the centroid of star image in the SATU CCD (Stellar Tracking Unit), which enables calculation of the wavelength assignment.

Wavelength assignment $(2 \mathrm{~Hz})$ : this is the wavelength of the center of each pixel, for the given measurement of transmission (it may change during one occultation owing to imperfect tracking). 
Geolocation $(2 \mathrm{~Hz})$ : this includes both the position of the ENVISAT spacecraft and the position of the tangent point of the LOS.

Limb products $(2 \mathrm{~Hz})$ : this product includes the background actually measured with the two external bands of CCD spectrometers, uncorrected and corrected for stray light and other factors. This product is found in separate dedicated files.

\subsection{Level 0 to Level $1 \mathrm{~b}$ processing details}

\section{Geolocation of the LOS}

In principle, the tangent altitude of one line of sight is defined only by the position of ENVISAT and the direction of the star. Several effects must be taken into account. The direction of the stars depends on the date because of the proper motion of the star and the aberration of light. Also, the LOS is not a straight line, because of atmospheric refraction. A full refraction computation is performed, assuming that the atmosphere is well represented by ECMWF predictions (for Near Real Time processing) or ECMWF analysis (for off-line processing, and re-processing).

\section{Wavelength assignment for spectrometers}

In this operation each pixel is assigned a wavelength. The assignment relies on regular calibration observations of stellar features, supplemented for Spectrometer B by the use of the $\mathrm{O}_{2}$ band atmospheric absorption, very well defined. Since the entrance slit is rather wide, equivalent to $\sim 10$ spectral pixels, the mean position of the star during one $0.5 \mathrm{~s}$ sample in the horizontal direction translates into a small wavelength shift that is taken into account. The star moves by a few microradians because of scintillations and imperfections of the tracking. Data from the SATU are used to correct the wavelength of each spectral pixel (spectel) by a small amount, since the image of the star is much smaller than the width of the entrance slit of the spectrometer.

\section{Bad pixels and cosmic ray processing}

A list of bad pixels is regularly updated from a GOMOS inflight calibration process called CALEX, which includes the use of a Uniformity monitoring mode. Only a few pixels, out of 2336, are out of order after 7 years in flight. The CCD detectors are sensitive to high energy particles arriving mainly from the Earth's radiation belts, at high latitude, and in the South Atlantic Anomaly. A cosmic ray produces an additional charge in a single pixel or in several pixels if the angle of incidence with respect to the surface of the CCD is small. This phenomenon is seen in all space-flying CCDs. A pseudo-image is built from the succession of all spectra in one single occultation, on which a median filter is applied to detect these cosmic rays. The pixel data are flagged and not used in the further processing. In bright limb condition cosmic ray processing is disabled.

\section{Modulation correction}

The offset level of the ADC (Analog to Digital Converter) was found to vary by 0.5 to $1.5 \mathrm{ADU}$ (Analog to Digital Unit), according to a very regular pattern. This pattern was determined, and a correction is now systematically applied for spectrometers A1 and A2.

\section{Conversion of ADU to measured electrons}

Each ADU value is converted to a number of measured electrons, for which Poisson statistics apply. A linearity correction is applied, which is verified at each CALEX monthly operation, by using a mode in which the same star is observed with various integration times from 0.25 to $10 \mathrm{~s}$. The correction depends on the CCD, but is quite small (less than $1 \%)$.

\section{Dark charge correction}

Even in the absence of light some electrons are generated in a pixel, the so-called Dark Charge, and must be subtracted to get the number of electrons generated by light. As described earlier, the CCDs have aged rapidly on this respect, and have increased their DC generation. As a result, the DC has increased constantly with time (Fig. 18); furthermore, it changes from one pixel to the next, and the DCNU pattern (Dark Charge Non-Uniformity) is changing over time scales of tens of minutes. The DCNU pattern is measured on each orbit, while observing a sky dark area, around the ascending node of ENVISAT (in the deep night). These measurements are used to correct all other occultations in the same orbit, with a multiplication factor taking into account the CCD temperature dependence of this DC. However, this temperature is digitized by steps of $0.4^{\circ} \mathrm{C}$, rather large to make a very accurate correction. In the future reprocessing, we will use the pixels in the UV range below $280 \mathrm{~nm}$ at low altitude, where we know that ozone blocks all the light, to determine the mean value of the DC, and the accurate variation of temperature between the DC calibration near the equator and the current measurement.

\section{Correction of the SFA mirror reflectivity}

The plane mirror of the SFA (Steering Front Assembly) is oriented in such a way that the line of sight is reflected in a fixed direction, along the telescope axis. The reflectivity of the mirror varies with wavelength and the angle of incidence, which is $\geq 25^{\circ}$. The incidence angle depends on the elevation and azimuth angles, defined in a frame of reference attached to ENVISAT. Since the incidence angle varies by a few degrees during one occultation, it is important 
to correct for the effect. Otherwise the transmission ratio of two star spectra measured with different incidence angles would be biased. A look-up-table has been built from Sirius observations with various azimuth and incidence angles. The variation of reflectivity is assumed to be a linear function of the angle of incidence, different for each wavelength. This effect was detected in flight, while monitoring the minute variations of star intensities: the influence of azimuth and elevation was obvious.

\section{Internal and external stray light correction}

At present time, no correction of the internal stray light (leakage of star light in the background bands) is implemented to correct the background bands. However, in full dark limb conditions, there is no subtraction of the background bands from the Central band where the star spectrum is recorded.

In some illumination conditions, there is some external stray light, either directly coming from the sun (and reflected on some part of ENVISAT and scattered to the mirror of GOMOS), or coming from the bright scene illuminated. Each occultation is qualified with an indicator (PCD ILLUM) defining the illumination conditions at the tangent point. One main parameter is the Solar Zenith Angle (SZA) at the tangent point. The data analysis may be slightly different for the various conditions. They are defined as follows:

PCD ILUM = 0: full dark

Not in bright limb nor twilight nor stray light conditions.

PCD ILUM = 1: bright limb

SZA at tangent point $<97^{\circ}$ for at least one measurement with altitude at tangent point $<50 \mathrm{~km}$.

PCD ILUM = 2: twilight

Not in bright limb and SZA at tangent point $<110^{\circ}$ for at least one measurement with altitude at tangent point $<100 \mathrm{~km}$.

PCD ILUM = 3: stray light

Not in bright limb and SZA of ENVISAT $<120^{\circ}$ for at least one measurement.

PCD ILUM = 4: twilight + stray light

Not in bright limb and both conditions for twilight and for stray light are verified. The index (PCD ILUM) may be also used as a guide for the stray light contamination.

\section{Vignetting correction}

The azimuth of the star may go from $-10^{\circ}$ to $+90^{\circ}$, where the origin $0^{\circ}$ is taken in the orbital plane. For negative azimuth ranging from -5 to $-10^{\circ}$, a part of the telescope area dedicated to feeding Spectrometer B is occulted by the plane mirror. A vignetting correction factor is applied for such cases.

\section{Correction of limb background}

In bright limb occultations the background radiation falling on the central band needs to be subtracted from the measured central signal in order to get the star signal alone. This background is estimated by a linear or exponential interpolation (the latter is currently activated in the processing) between the measurements of the background made in the upper (U) and lower (L) bands. The central background estimate is stored in the Level $1 b$ product. The estimates of the upper and lower background are stored in the GOMOS Limb Products, which may be analyzed to retrieve also ozone and other gases, as done for SCIAMACHY (Bovensmann et al., 1999) and OSIRIS on ODIN (Tukiainen et al., 2008).

Before the estimates, a flat field correction is applied. The sensitivity of each pixel is retrieved from a PRNU map (Pixel-to-pixel Response Non-Uniformity). The PRNU values of all pixels have been determined in-flight, and for the PRNU band correction an average is made over the 7 lines of the upper (U), central (C) and lower (L) bands.

The subtraction of the background estimate is not performed in the full dark limb conditions for Spectrometer A because it is negligible and subtracting it would add the noise of the Dark Charge of the two background bands. For spectrometer B (IR), it is not negligible, not even during the night. There are indeed an $\mathrm{O}_{2}$ emission around $760 \mathrm{~nm}$ and $\mathrm{OH}$ emissions near $940 \mathrm{~nm}$.

On the day-side, the limb brightness is large. It saturates the spectrometers usually at altitudes lower than $26 \mathrm{~km}$ so that the background correction is no longer feasible. When any of the upper, central or lower band is saturated, the corresponding column is flagged and not used in the ensuing processing.

\section{Star signal and transmission}

The stellar signal created by the background subtraction must be corrected for flat-fielding in order to get a valid estimate of the star spectrum. The nominal location of the star signal on the CCD, the SATU output data, the photometer engineering data and the PRNU full maps are used in flat-fielding. The flat-fielding is most important for spectrometers B1 and B2 (up to $\pm 20 \%$ for $\mathrm{B} 2$, due to an optical interference in the coating of the CCD, and of the order of $\pm 1-2 \%$ for $\mathrm{A} 1$ and A2). The exact position of the star communicated by the SATU is taken into account. In spite of these refinements, we found that the PRNU effect was not corrected perfectly for the $\mathrm{B} 2$ spectrometer, dedicated to $\mathrm{H}_{2} \mathrm{O}$ measurements at $936 \mathrm{~nm}$. We suspect that this is because the star light is extremely well focused on the CCD, and that the pixel response is not uniform over one pixel. For the next version of the processing, a new sub-pixel PRNU map will be used, which could be established when the plane mirror made unwanted excursions over the SATU detector (Bertaux et al., 2009). 
Once the star signal is corrected as described above to obtain $S_{\text {obs }}$, the 10 first spectra (obtained above $120 \mathrm{~km}$ ) are averaged together to form the reference spectrum $S_{\text {ref }}$ , and the atmospheric transmission $T_{\text {obs }}$ for each sample is calculated on a fixed reference wavelength grid (one value per spectral element, or "spectel") as the ratio:

$T_{\text {obs }}=S_{\text {obs }} / S_{\text {ref }}$

This is the actually measured transmission, not corrected for dilution nor scintillations. Therefore, in some extreme cases of scintillations, it may be larger than 1 . These corrections are done during Level 2 processing.

\section{Error bar on the transmission}

All Level $1 \mathrm{~b}$ products are accompanied by error estimates. These estimates are calculated assuming that there is no modeling error and normal Gaussian error statistics. The pixelwise data are assumed to be independent of each other. The error is estimated from Poisson statistics for the number of electrons, the CCD read-out noise, the digitization step (one ADU corresponds to different numbers of electrons for the various CCDs and operating modes) and the error of the background subtraction, as described in more details in Kyrölä et al. (2009). All these error estimation formulas have been validated by simulations and by in-flight statistical analysis of measurements.

\section{Photometer data processing to reach Level 1b}

The photometer data (red and blue channels) are measured with a sampling rate of $1 \mathrm{kHz}$ and each sample corresponds to a time integration of $1 \mathrm{~ms}$. The detectors are CCDs of $16 \times 16$ pixels, but only 14 lines are binned together, and five central columns only are read separately to procure a signal in ADU and added together digitally.

Because of the 12bits capacity of the on-board adder included in the photometer electronic chain, only the 12 least significant bits are written in the GOMOS packets. In case of overflow, the analysis of the temporal series usually allows rebuilding the original signal by continuity (unfolding algorithm). This algorithm may fail for Sirius (strongest signal) and in a case of strong scintillations.

There is also a Dark Charge correction and the total of ADUs after unfolding is converted into electrons in the processing with the appropriate gain.

A strong sub-pixel response is present in these small CCDs, as was discovered recently.

\subsection{Level 1b to Level 2 processing}

\subsubsection{Level 2 products and processing overview}

Starting from the Level $1 \mathrm{~b}$ products, the Level 2 processing aims at computing the following Level 2 products for each tangent altitude measurement:
- The local densities (or concentrations in $\mathrm{cm}^{-3}$ ) of ozone, $\mathrm{NO}_{2}, \mathrm{NO}_{3}, \mathrm{OClO}, \mathrm{O}_{2}, \mathrm{H}_{2} \mathrm{O}$ and their error bars.

- The line densities (also called slant densities, $\mathrm{cm}^{-2}$ ) of the same species and their error bars, which are the concentrations integrated along the line of sight.

- The aerosol extinction and its wavelength dependence.

- The temperature with a high vertical resolution (available in the next re-processing).

- The geolocation of these data.

- The air density given in the ECMWF/MSISE-90 model, and the corresponding integrated line density along the LOS. This product obviously does not come from GOMOS data, but is recommended for use when one wants to compute a mixing ratio from GOMOS density products.

Each of these products gives one vertical profile for each stellar occultation, with about 30-40 occultations per orbit.

The processing is divided into two sequential steps:

1. The spectral inversion, which gives the line densities for a given transmission measurement.

2. The vertical inversion, which provides the local densities from the line densities.

This second step assumes that the distribution of species has a locally spherical symmetry, while the extraction of line densities are independent of such an assumption. In addition, the local density data are noisier than the line densities, because the vertical inversion introduces some oscillations that are smoothed out by a Tikhonov regularization, decreasing somewhat the vertical resolution. Therefore, the time variations and long term trends may be studied, not only on the local densities, but also directly with the line densities.

The spectral inversion is quite different for spectrometer $\mathrm{A}$ and $\mathrm{B}$, and they are treated almost fully separated. The reason is that in the UV-VIS domain of spectrometer A, the forward modeling of the transmission is rather fast, and is used in a best fit approach with Levenberg-Marquardt scheme; while for $\mathrm{O}_{2}$ and $\mathrm{H}_{2} \mathrm{O}$ absorption calculations, forward modeling requires a line-by-line computation with the HITRAN data base, with a prohibitive computing time if done on-line.

The GOMOS Level 2 data processing assumes that a measured transmission, $T_{\mathrm{obs}}$, (produced by the GOMOS Level $1 \mathrm{~b}$ processing) can be taken as a product of two transmissions:

$T_{\text {obs }}=T_{\text {ref }} T_{\text {ext }}$

The transmission $T_{\text {ref }}$ is due to refractive effects and the transmission $T_{\text {ext }}$ is due to absorption and scattering 
processes in the atmosphere. The Level 2 processing aims first at estimating the refractive part using the fast photometer data and the geolocation data, and then to remove it from the measured transmission. The remaining transmission $T_{\text {ext }}$ can then be connected to the atmospheric constituent densities, the retrieval of which is the main mission objective of the GOMOS instrument. This transmission can be written as:

$T_{\text {ext }}=e^{-\tau}$

where the optical thickness $\tau(\lambda)$ is given by an integral along the line of sight $s$ :

$\tau(\lambda)=\sum_{j} \int \sigma_{j}(\lambda, r(s)) \rho_{j}(r(s)) d s$

in which $\rho_{j}$ is the density of species $\mathrm{j}$, with absorption cross section $\sigma_{j}(\lambda)$ and $r(s)$ is the altitude of the running point with curvilinear abscissa $s$ along the line of sight. The simplifications discussed in Kyrölä et al. (2010a) lead to some inaccuracies of the modeling but they are counteracted by performing iteration loops. After performing spectral and vertical inversion we perform the sequence again and so on. Usually one iteration is enough to converge.

In the forward model that computes the model transmission to be compared to $T_{\text {ext }}$, one must take into account the finite measurement time $(0.5 \mathrm{~s})$ of GOMOS. During this time the tangent point altitude can change by up to $1.7 \mathrm{~km}$, for occultations in the orbital plane. For occultations outside the orbit plane this movement is smaller, leading to a better vertical sampling resolution.

Also, because of the finite spectral resolution of the instrument, we cannot simply use the cross-sections as such in the inversion. One solution often chosen is to convolute the cross-sections by the instrumental profile, which is mathematically incorrect. Rather, we convolute the model transmission by the instrument profile.

\subsubsection{Refraction corrections}

The scintillation and dilution correction process is based on the assumption that the modulation due to refraction may be split into two independent terms:

$T_{\text {ref }}=T_{\text {dil }} T_{\text {sci }}$

where $T_{\text {dil }}$ represents the dilution component and $T_{\text {sci }}$ represents the effect of scintillation. The dilution is due to the fact that refraction bends the star light rays more and more as the impact parameter decreases. It depends on the derivative of the refractive index with altitude, and on the distance of the observer to the limb. This is one reason why the stellar occultation is not very appealing from a geostationary orbit.

The scintillation is derived from the fast fluctuations of the photometers, assuming that the fluctuations due to extinction by species are negligible at these small vertical scales, which is justified by the geometry of the line of

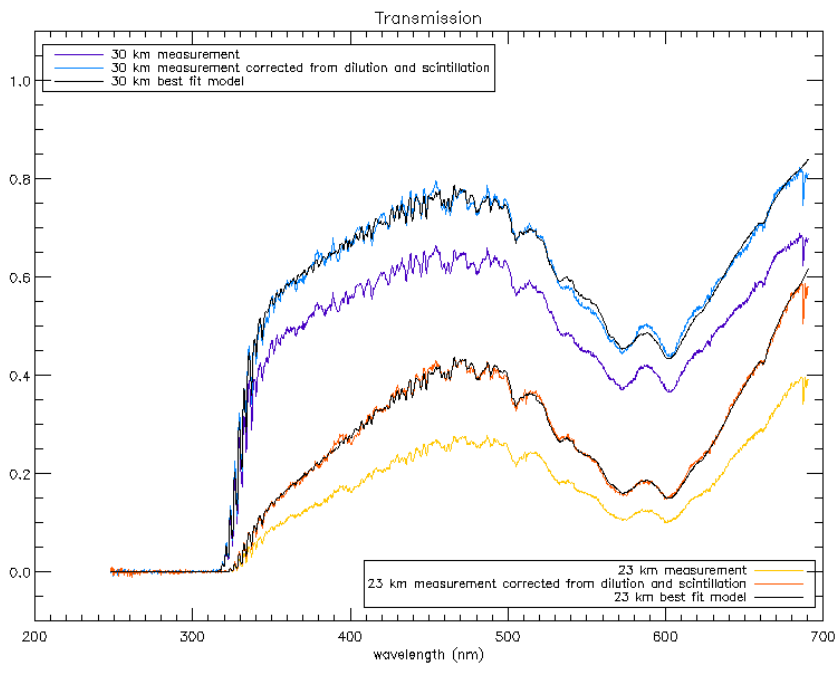

Fig. 21. Examples of transmissions measured at 23 and $30 \mathrm{~km}$. The Level $1 \mathrm{~b}$ measurements are in yellow and violet for 23 and $30 \mathrm{~km}$, respectively. They are shown in red and blue after correction for dilution and scintillations, while the best fit models of the transmission are shown in black.

sight, integrating over $\sim 300 \mathrm{~km}$. Once $T_{\text {dil }}$ and $T_{\text {sci }}$ are determined, the observed transmission $T_{\mathrm{obs}}$ is divided by $T_{\text {ref }}$, to obtain the transmission due only to the extinction spectrum $T_{\text {ext }}$ (Fig. 21).

Since the refraction depends on the wavelength, one transmission spectrum probes slightly different altitudes. A correction for chromatic refraction is made by means of a linear interpolation between two consecutive acquisitions and this corrected transmission is then fit by a forward model of the transmission that depends on all the species that we want to determine.

\subsection{Spectral inversion of spectrometer A (UVIS)}

\subsubsection{Cross sections}

As described in more detail in Kyrölä et al. (2010a), some refinements are introduced to take into account the finite integration time of the instrument, the finite spectral resolution of the imaging system (as well as the combined of effects of these two processes) and the temperature-dependent value of the cross-section (important for UV absorption of ozone). The cross-sections and their temperature dependence are taken from the GOMOS data base (Figs. 2 and 3).

\subsubsection{Aerosols}

Unlike the other trace gases retrieved by the spectral inversion of GOMOS transmission data, the slant path optical thickness, $\tau_{\mathrm{a}}(\lambda)$, of the aerosols and of the stratospheric or mesospheric clouds is not characterized by a well 
defined extinction cross-section that can be measured in the lab. Instead, the spectral dependence of $\tau_{\mathrm{a}}(\lambda)$ is a product of the inversion, not data. In the UVvisible range, the extinction of light results from the Mie scattering by a population of particles whose size distribution is a priori unknown. Important diffractive effects are expected when the wavelength matches the characteristic particle size. However, these effects are smoothed out when integrated over an extended particle size distribution, allowing expression of $\tau_{\mathrm{a}}(\lambda)$ as a smooth (polynomial) wavelength dependence as:

$\tau_{\mathrm{a}}(\lambda)=\tau_{\mathrm{a}}^{[0]}+\tau_{\mathrm{a}}^{[1]}\left(\lambda-\lambda_{\text {ref }}\right)+\ldots \tau_{\mathrm{a}}^{[p]}\left(\lambda-\lambda_{\text {ref }}\right)^{p}$

and the spectral inversion consists of retrieving the quantities $\tau_{\mathrm{a}}^{[0]}, \tau_{\mathrm{a}}^{[1]}, \ldots \tau_{\mathrm{a}}^{[p]}$.

The present stratospheric transparency is very high due to the quasi-unperturbed relaxation of the Pinatubo aerosol load since 1991, which makes the measurement of stratospheric aerosols a difficult task. Therefore we restricted the description of the aerosol slant path optical thickness to a form with $\sigma_{0} / \lambda$ wavelength dependence in the current operational version of the algorithms, GOPR 6 (also called IPF 5).

During volcanic periods like the aftermath of the Mount Pinatubo eruption (Fussen et al., 2001) large stratospheric particle formation has been observed up to radii of $0.5-$ $0.7 \mu \mathrm{m}$ that induced a broad maximum in the optical wavelength dependence. The wavelength dependence was modified for the new version GOPR 7 (or IPF 6), by taking a quadratic form to describe $\tau_{\mathrm{a}}(\lambda)$. The arbitrary reference wavelength has been fixed at $\lambda_{\text {ref }}=500 \mathrm{~nm}$ after an empirical optimization of the covariance matrix elements. Tamminen et al. (2010) have studied the influence on ozone line density retrieval of taking various forms of the aerosols wavelength dependence. There are some significant differences below $20 \mathrm{~km}$.

\subsubsection{Air density}

Before launch we thought that the air density could be retrieved from its Rayleigh extinction. However, because of coupling with aerosol extinction and $\mathrm{NO}_{2}$ cross-section, the achieved accuracy was not satisfactory. We decided to fix the neutral density to the value given by ECMWF for altitudes below $1 \mathrm{hPa}$ and to MSISE-90 model above. It should be emphasized that ECMWF outputs are based on actual measurements made all over the planet, updated every $6 \mathrm{~h}$ through data assimilation, therefore providing a good representation of reality.

\subsection{4 $\mathrm{NO}_{2}$ and $\mathrm{NO}_{3}$}

In the $20-40 \mathrm{~km}$ altitude range, chromatic scintillations caused by isotropic turbulence create perturbations in GOMOS transmission spectra. These lead to unrealistic oscillations in the vertical profiles of the line densities of $\mathrm{NO}_{2}$ and $\mathrm{NO}_{3}$. The scintillation correction step is unable to remove these scintillations from the transmission data. In order to improve the retrieval, a variant of DOAS (Differential Optical Absorption Spectrum) named GDI (Global DOAS Iterative) is applied for $\mathrm{NO}_{2}$ and $\mathrm{NO}_{3}$.

In the GDI a species is artificially separated into two virtual species, $X_{S}$ and $X_{D}$, with their respective crosssections, one which has a cross-section varying smoothly with wavelength, the other having a rapidly varying crosssection, the sum of the two cross section being equal to the total cross section of $\mathrm{NO}_{2}\left(\right.$ or $\left.\mathrm{NO}_{3}\right)$.

The spectral inversion is performed in two phases. The first Levenberg-Marquardt fit is applied with $X_{S}=0$ and $X_{D}$ as a free parameter together with all other species that we want to retrieve. The fit gives a first value $X(1)_{D}$. The second fit is applied with $X_{S}=X(1)_{D}$. A second value $X(1)_{D}$ is obtained. The process is iterated until the maximum number of iterations is performed or until a given convergence criterion is reached.

\subsection{Spectral inversion of IR spectrometer $B$ $\left(\mathrm{O}_{2}\right.$ and $\left.\mathrm{H}_{2} \mathrm{O}\right)$}

The IR spectrometer measures the densities of two different constituents $\mathrm{O}_{2}$ and $\mathrm{H}_{2} \mathrm{O}$ in two bands: $756-774 \mathrm{~nm}$ (spectro B1) and 926-954 nm (spectro B2), respectively. Due to the physics of the problem, the individual absorption lines are very narrow and saturated, the apparent crosssections change with the integrated densities, and they can no longer be considered as only wavelength dependent. The calculation of the transmission function is computationally very demanding. Therefore, it is difficult to use the same direct inversion method as for the UVIS spectrometer data.

A new algorithm has been developed. The method uses reference transmission spectra, $T_{\bmod }(\lambda, N)$, that are calculated for different integrated line densities, $N$, of $\mathrm{O}_{2}$ and $\mathrm{H}_{2} \mathrm{O}$. These reference transmissions depend on pressure and temperature. To take into account this dependency, calculations of transmission are performed by using a direct model with a standard atmospheric profile. Several such calculations are done for different atmospheric models (US Standard 76) in order to get the response for 5 different conditions such as tropical, mid-latitude (winter and summer) and subarctic atmospheric profiles. The calculations were made in steps of $1 \mathrm{~km}$ altitude from 0 to $100 \mathrm{~km}$, with the highest possible wavelength resolution allowed by the HITRAN data base, and the radiative transfer code LBLRTM (http://rtweb.aer.com/lblrtm.html). Then, they are convoluted with the spectral function of the instrument and integrated over each spectel wavelength interval $(0.046 \mathrm{~nm}$ for B1 and 0.056 for B2). They constitute transmission model tables, or LUT (Look Up Tables). 
For each measured transmission, $T_{\mathrm{obs}}$, at an altitude, $z$, and for the climatic table appropriate to latitude and season, a scatter plot of $T_{\mathrm{obs}}$ as a function of $T_{\bmod }(\lambda, N)$ is made for all the pixels of B1 (or B2) separately in log coordinates, for a number of altitudes $z_{i}$ (and corresponding $N_{i}$ ), around the altitude of measurement. A best-fit linear fit yields a value of the slope $a_{i}$; in the series of $a_{i}$, two consecutive values bracket the value 1. A linear interpolation for $a=1$ is then done in the series $a_{i}, N_{i}$, yielding the retrieved best-fit line density $N_{\text {ret }}$.

In order to take into account a possible residual continuous extinction and its possible wavelength dependence, the fitting function of the scatter plot is of the form:

$\ln \left(T_{\mathrm{obs}}\right)=a \ln \left(T_{\bmod }(\lambda, N)\right)+b / \lambda+c$

and the parameters $b$ and $c$ are also retrieved in the process. Actually, for the $\mathrm{O}_{2}$ band at $760 \mathrm{~nm}$, there is a small absorption by ozone in the Wulf bands. Therefore, the observed transmission $T_{\text {obs }}$ is first corrected from the extinction of ozone, as determined from the spectral inversion of spectrometer A. In addition, the Rayleigh extinction by air (fixed with ECMWF and MSISE-90), and the aerosol extinction extrapolated at the wavelength of spectrometers B1 and B2 are also computed for a further correction of $T_{\mathrm{obs}}$, before the comparison with the tables of $T_{\text {mod. }}$.

The values of $b$ and $c$ are not used at present. They may represent a deviation of the wavelength dependence of aerosol extinction far away from the reference wavelength $(500 \mathrm{~nm})$. However, one should be cautious, since the dilution and scintillations may change quite severely the level of the continuum, and though the processing does try to correct for it, the value of $c$ may not represent only the aerosol extinction. At any rate, the value of a is independent of $c$. Due to its nature, this method has been called "comparative method".

\subsection{Vertical inversion}

The GOMOS vertical inversion seeks finding a vertical profile that, when integrated along the various lines of sight during one occultation, gives the measured line densities, $N_{i}$. This is the inversion of Abel's integral. The number of layers is given by the number of measurements in the occultation processed. The resulting problem is a readily solvable linear inversion problem. By the triangular nature of the kernel matrix the inversion can be done using the so-called onion peeling method. While in its standard version, the onion peeling technique yields layers with constant density in each layer, for GOMOS we approximate the local densities to be linear as a function of altitude between two successive GOMOS measurements.

The fact that line densities are an average over the integration time is dealt with a Taylor expansion. Unphysical oscillations attached to the classical problem of inversion of
Abel's integral are smoothed by a special type of Tikhonov regularization (Sofieva et al., 2004). The parameters of such a regularization were selected to provide a constant vertical resolution over some range of altitudes: 20 to 30,30 to 40 and above $40 \mathrm{~km}$. The resolution sought is different for each species and each altitude region. They are indicated below for each species, in the 3 above-defined altitude regions (separated by commas, in order of increasing altitude:

$$
\begin{aligned}
& \mathrm{O}_{3}: 2,2-3,3 \mathrm{~km}, \\
& \text { Aerosols, } \mathrm{NO}_{2}, \mathrm{NO}_{3}, \mathrm{OClO}: 4,4,4 \mathrm{~km}, \\
& \mathrm{O}_{2}: 3,3-5,5 \mathrm{~km}, \\
& \mathrm{H}_{2} \mathrm{O}: 2,4,4 \mathrm{~km} .
\end{aligned}
$$

Once the vertical inversion is done, the spectral inversion is performed once more in order to take into account the temperature dependence of the cross sections. This loop is necessary because temperature-dependent cross sections in the extinction line-of-sight integrals (Beer-Lambert formula) couple the spectral and vertical inversion problems.

\subsection{Error estimate}

With GOMOS we measure atmospheric transmissions, from which are derived optical thicknesses from the BeerLambert law. Optical thicknesses are absolute quantities; consequently all errors affecting the measurements translate into absolute errors in line densities, then in local densities after vertical inversion. Relative errors are then computed by the ratio of absolute errors to retrieved absolute quantities (line densities, or local densities as shown on Figs. 14 and 22). The absolute error estimate is based on the propagation of all Level $1 \mathrm{~b}$ errors, the largest contributors to which are dark current and photon noise, through the spectral and vertical inversions. An additional error source in Level 2 is the step where intensity fluctuations from scintillations are removed using photometer data. In the currently available GOMOS data (IPF 5, GOPR 6), this additional error is estimated empirically from large numbers of equatorial measurements with varying azimuth angle. The underlying assumption is that the ozone distribution around the equator is relatively constant and the variability of ozone profiles comes from scintillations. In the new version of the processing, GOPR 7.0, this is replaced by the use of a full co-variance matrix between spectral pixels: scintillations are perturbing the spectrum, with a strong covariance between adjacent pixels. The retrieved quantities are not different from the previous version GOPR 6 , but the error bars are somewhat reduced, and are much better estimated. Relative errors are computed as the ratio of absolute errors to the derived absolute quantity.

Figure 22 (from Tamminen et al., 2010) shows the new error estimate for ozone and $\mathrm{NO}_{2}$, for various stars (brightness and temperature), and for vertical or oblique 


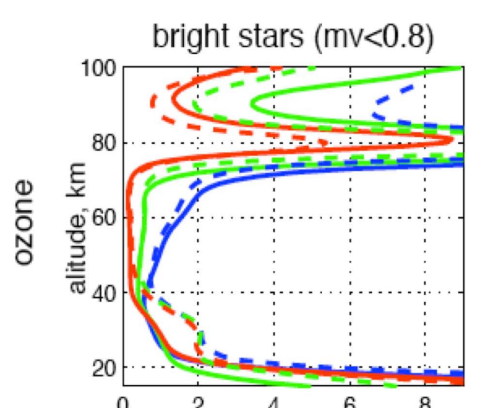

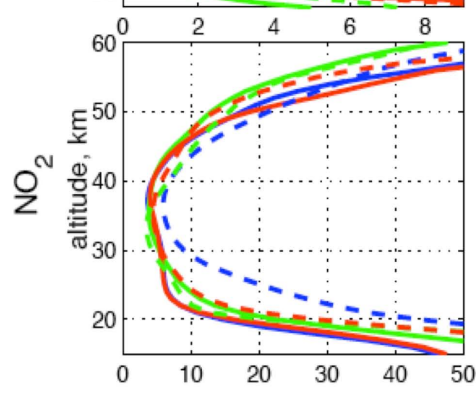

relative error estimate $\%$
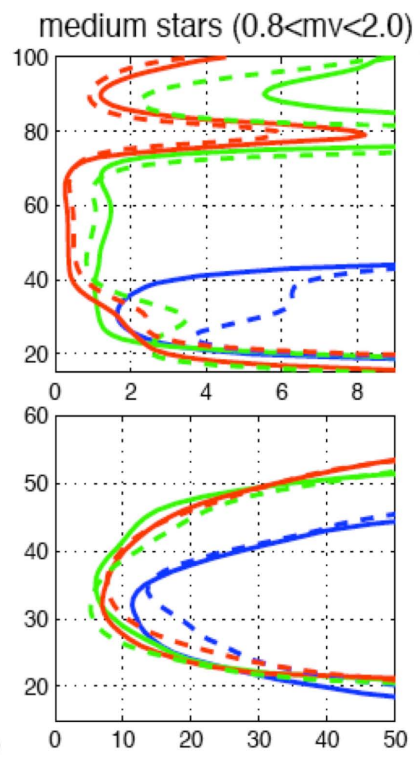

relative error estimate, \%
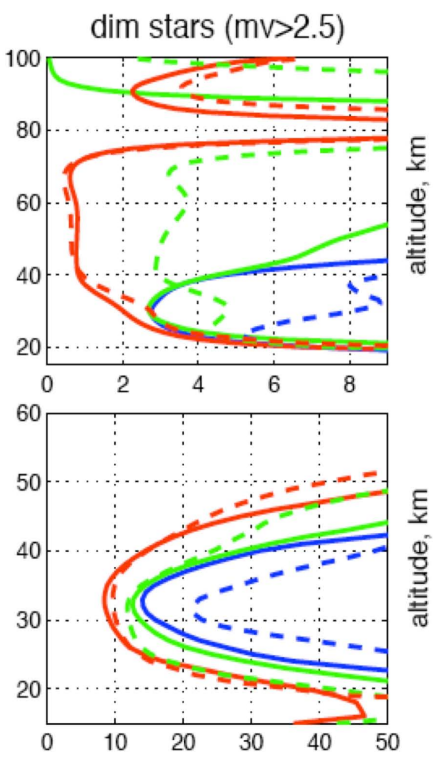

relative error estimate, $\%$

$$
\longrightarrow V, \text { cool } \longrightarrow V \text {, medium } \longrightarrow V \text {, hot }=-=0 \text {, cool }=-=0 \text {, medium }=-=0 \text {, hot }
$$

Fig. 22. GOMOS error estimates for $\mathrm{O}_{3}$ (top row), and $\mathrm{NO}_{2}$ (second row) for representative cases: bright star (left column), typical star (middle column) and dim star (right column). The dashed lines correspond to oblique occultations and the solid lines to occultations in the orbital plane. Stellar temperature is indicated with the line color: hot stars (red), medium stars (green) and cool stars (blue). These values correspond to the most recent version of data processing (GOPR 7.0), which is the baseline for the reprocessing in 2010 (from Tamminen et al., 2010).

occultations (the latter being more sensitive to scintillation errors). For ozone the retrieval error of the night-time measurements is typically $1-5 \%$ in the stratosphere, and better than $8 \%$ up to $100 \mathrm{~km}$, except for cool stars above $40 \mathrm{~km}$. The precision between 20 and $40 \mathrm{~km}$ is around 1$3 \%$ and below $20 \mathrm{~km}$ it degrades to $10 \%$ at $15 \mathrm{~km}$. With reasonably bright stars $\mathrm{NO}_{2}$ can be measured at $20-50 \mathrm{~km}$ altitude range with $10-20 \%$ precision. $\mathrm{NO}_{3}$ can be measured in the $25-45 \mathrm{~km}$ altitude range with a precision of 20 $40 \%$ with bright and medium brightness stars. Aerosols can be measured up to $30 \mathrm{~km}$ with about $5-10 \%$ precision. Below $15 \mathrm{~km}$ the accuracy degrades to $30 \%$. Tamminen et al. (2010) have also shown that the increasing CCD dark charge (Fig. 18) degrades the ozone accuracy from $1.5 \%$ to $2.8 \%$ (2008) for a dim star $\left(n^{\circ} 124\right)$, while the degradation is weak or negligible for bright stars.

\subsection{High resolution temperature profile}

Besides the scintillation correction, the photometers' signals provide a way to study small scale structures in the atmosphere. A high resolution temperature profile can be derived from the time delay between the two photometers. This is one of the GOMOS Level 2 products for IPF 6 . The algorithm is based on the computation of the difference in time delay between the signals of the two photometers. Due to the variation of the index of refraction of air with wavelength, the light beam of an occulted star is more bent in the blue part of the spectrum than in the red part. For a given tangent altitude, the red beam reaches GOMOS before the blue beam (see Fig. 17). The computation of the time delay gives information on the bending angle, which is related to the density and temperature profiles in the atmosphere. This method enables derivation of a high resolution temperature profile with a vertical resolution of $100-200 \mathrm{~m}$, in the altitude range $\sim 20-40 \mathrm{~km}$, where scintillations are most pronounced (see paragraph 7.15).

\section{Validation of GOMOS products}

In principle, the star occultation method is self-calibrating, and does need to be validated, since an absolute quantity is derived from a relative measurement. The reality is more complex (in a large part due to scintillations) and the comparison with other sources of similar data (validation exercises) makes it possible to adjust the processing, or even to detect errors. One good example is the retrieval of $\mathrm{H}_{2} \mathrm{O}$, which showed a systematic maximum of $\sim 9 \mathrm{ppmv}$ at $29 \mathrm{~km}$, while other instruments never showed such a behaviour. This 


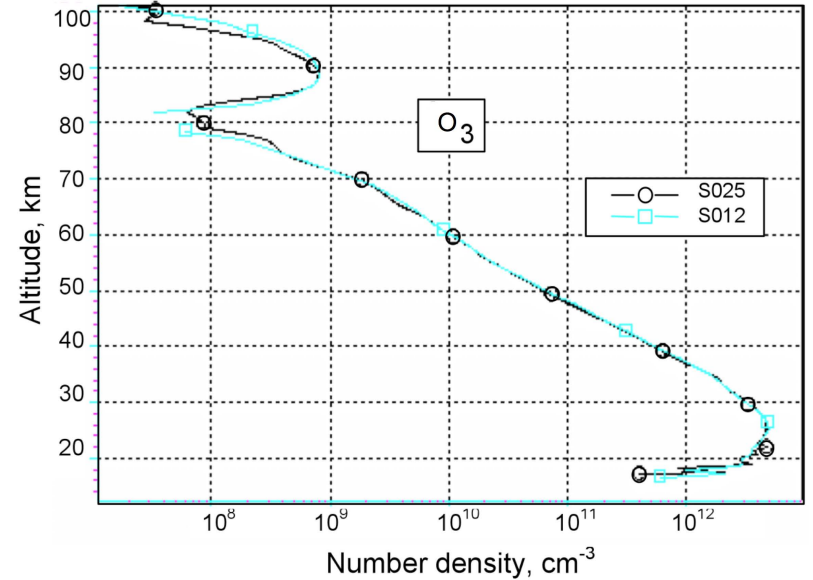

Fig. 23a. Two vertical profiles of ozone obtained at the same geographical location, but on two successive orbits and using two different stars with the tangent points differing by only $25 \mathrm{~km}$.

bias could be traced back to an unexpected strong sub-pixel PRNU of spectrometer B2 (Bertaux et al., 2009).

The GOMOS data validation activity has been carried out since the summer of 2002. The most useful comparison for ozone was made by Meijer et al. (2004), with balloon-borne ozone sondes, lidars and microwave instruments, which all produce vertical profiles like GOMOS. The results show that in dark limb the GOMOS data agree very well with the other data: between 14 and $64 \mathrm{~km}$ altitude their differences show only a small $(2.5-7.5 \%)$ insignificant negative bias with a standard deviation of $11-16 \%(19-63 \mathrm{~km})$. This conclusion was demonstrated to be independent of the star temperature and magnitude and the latitudinal region of the GOMOS observation, with the exception of a slightly larger bias in the polar regions at altitudes between 35 and $45 \mathrm{~km}$. GOMOS, MIPAS and SCIAMACHY measurements of $\mathrm{O}_{3}$ and $\mathrm{NO}_{2}$ have been compared by Bracher et al. (2005). In the mesosphere and lower thermosphere only satellite data are available for validation purposes. GOMOS ozone results have been compared with MIPAS by Verronen et al. (2005a). A general agreement between these two instruments has been found, but at that time too few coincident measurements were available for a definitive comparison. Another ozone profiles comparison was made in the tropics with $\mathrm{SAOZ}$ long-duration balloon measurements (with solar occultation measurements) in 2003 and 2004 (Borchi and Pommereau, 2007), together with other instruments. A good agreement was found between 22 and $26 \mathrm{~km}$ showing a small negative bias $(-1$ to $-2.5 \%)$ and $6 \%$ dispersion on individual profiles.

In the case of GOMOS $\mathrm{NO}_{2}$, validation against MIPAS has indicated a good night-time agreement between the two instruments (Verronen et al., 2009). In the middle and upper stratosphere the statistical differences were found to be within 5 and $15 \%$, respectively. For high mesospheric
$\mathrm{NO}_{2}$ mixing ratios observed in the winter polar regions, the differences were within $35 \%$. In the vast majority of cases, the differences were smaller than the combined systematic error of the measurements, giving great confidence in the data quality.

One way to check the internal consistency of the GOMOS data and retrieval scheme is to compare the vertical profile of ozone obtained at the same geographical location, but at two different (successive) orbits, and using two different stars (at 2 consecutive orbits, the same star is occulted at the same latitude, but at longitudes differing by about $25^{\circ}$ ). This is shown in Fig. 23a, where the tangent points of two consecutive orbits differed by only $25 \mathrm{~km}$. The two vertical profiles are in excellent agreement and show also the unique capability of GOMOS to measure mesospheric ozone at night. It should be noted that while it is assumed, in the vertical inversion, that the ozone distribution is locally spherically symmetric, in reality there are horizontal variations of the local density. As a result, the line density integrated at a given tangent altitude may depend on the angle of the line of sight (with the meridian circle, for example). Calculations have shown that there may be $4 \%$ variation in a modeled ozone 3-D field, and this may account for some of the small differences seen in the two profiles of Fig. 23a.

Another way to check the GOMOS internal consistency is to examine a series of profiles in a region where natural variability is in principle small. This is the case for low latitudes, as illustrated in Fig. 23b, showing (left) a series of 10 profiles obtained with the star Sirius, gathered within a day (therefore, at a constant latitude of $9^{\circ} \mathrm{N}$, and sampling all longitudes). While above $30 \mathrm{~km}$, all measurements are almost identical, below $30 \mathrm{~km}$ there are longitude variations of the detailed vertical structure; daily mean profiles may be constructed, and superimposed together as in Fig. 23b (right) showing 19 of such daily mean profiles. During this span time, the point of star occultation varied from 0 to $15^{\circ} \mathrm{N}$, which may be a factor for the small internal dispersion of the daily profiles.

\section{Overview of GOMOS results}

\subsection{Ozone climatology in the stratosphere}

Before GOMOS on ENVISAT, the most commonly used ozone climatology with vertical profiles (based on measurements) was produced by Fortuin and Kelder (1998) (hereafter, F-K), primarily based on ozone sondes up to $30 \mathrm{~km}$, and satellite data above $30 \mathrm{~km}$ from SBUV and SBUV-2, using nadir back-scattered solar UV, which have an inherently coarse vertical resolution, and stopping at $0.3 \mathrm{hPa}$ $(\sim 55 \mathrm{~km})$.

GOMOS was able to provide the first climatology of ozone extending up to $100 \mathrm{~km}$, thus covering the mesospheric ozone layer, and with a good vertical resolution. It was produced 

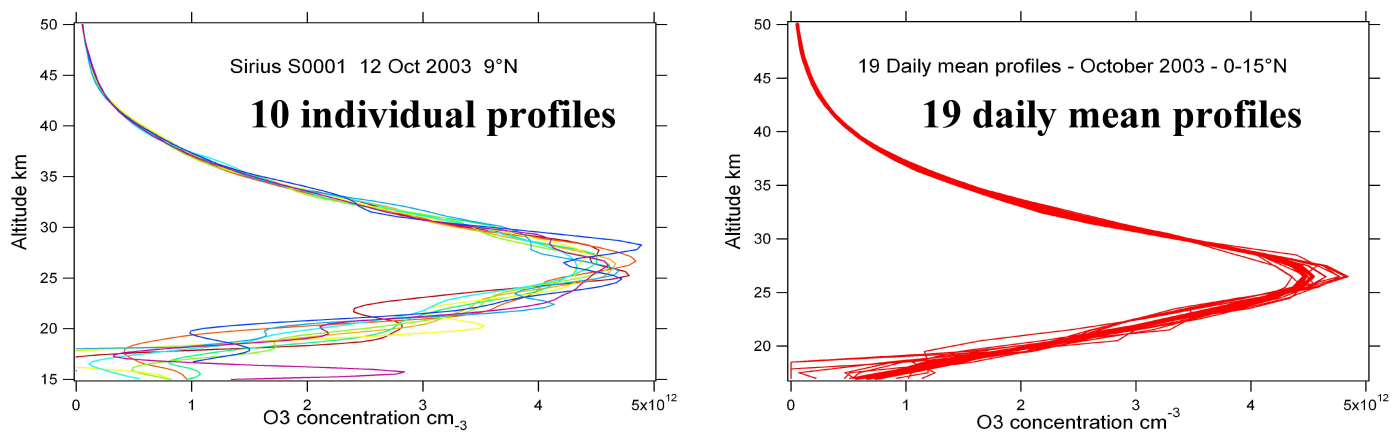

Fig. 23b. Left: ozone vertical profiles obtained with the occultation of Sirius at $9^{\circ} \mathrm{N}$ and 10 different longitudes, obtained in a single day ( 9 October 2003). Right: 19 daily mean profiles obtained with Sirius during the month of October 2003.

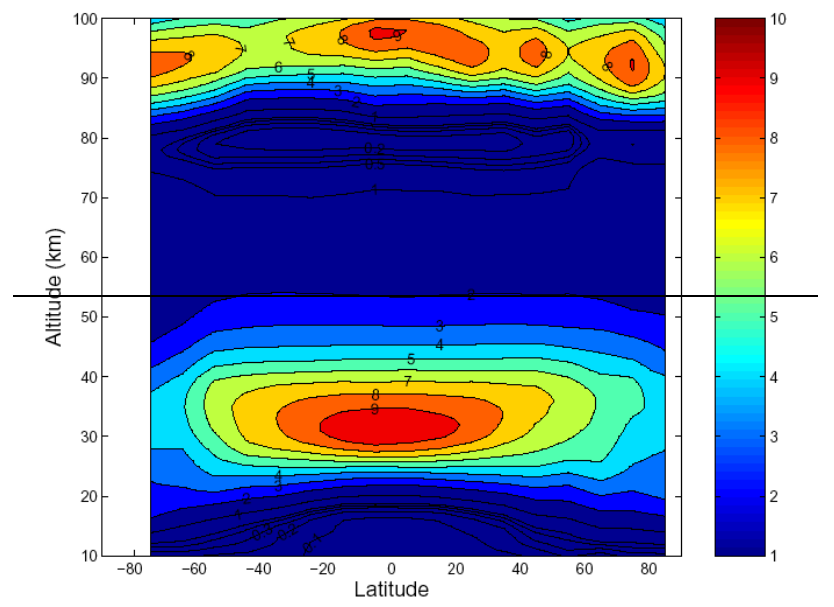

Fig. 24. Ozone mixing ratio yearly mean from GOMOS night time occultations only (ppmv). Polar areas are not sampled during the summer and only the region $50^{\circ} \mathrm{S}$ to $50^{\circ} \mathrm{N}$ are sampled over the whole year.

from all night-time star occultations recorded during the year 2003 (Kyrölä et al., 2006). The night-time limit was set at a solar zenith angle of $108^{\circ}$, and 43000 occultations were kept in the analysis, binned by month and latitude zone of $10^{\circ}$ (zonal average). The quality was controlled by checking the retrieved ozone profiles for each star individually. The altitude range covered from 10 to $100 \mathrm{~km}$, covering the whole stratosphere and the mesosphere lower thermosphere (MLT) at the same time. For the cool stars (with no UV flux), the maximum valid altitude was 35 to $45 \mathrm{~km}$. Mixing ratios were computed with air densities from ECMWF below $50 \mathrm{~km}$, and MSISE-90 model above, included in the GOMOS products, and the yearly mean is represented on Fig. 24.

This 2003 climatology is compared (Fig. 25) to the earlier F-K stratospheric climatology, using the yearly average. In the upper stratosphere above $45 \mathrm{~km}$, GOMOS shows more ozone by $5-10 \%$ (increasing with altitude), explained by the diurnal cycle of ozone at these altitudes: GOMOS measures

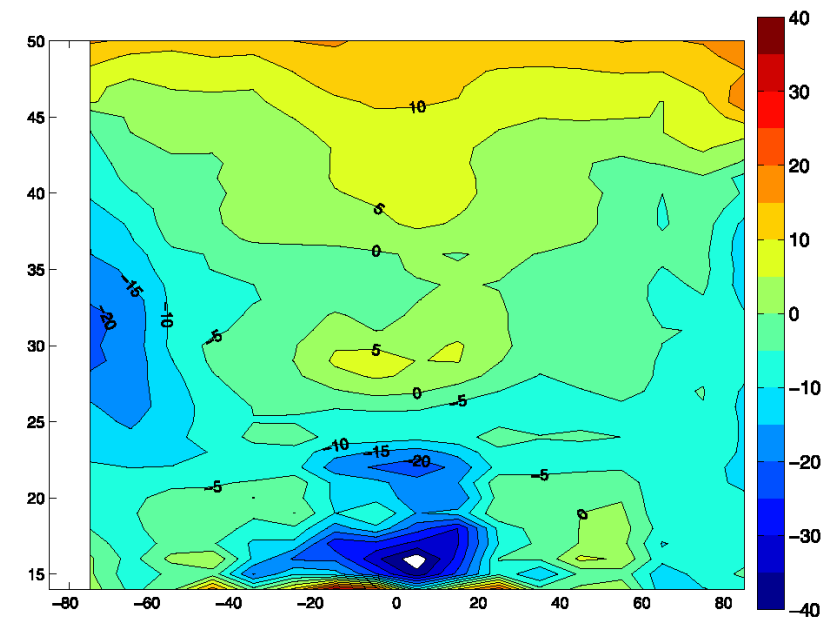

Fig. 25. Median of the relative difference (in\%) between GOMOS ozone mixing ratio and the F-K climatology in 2003. The contours are separated by $5 \%$. The horizontal axis is latitude in degrees, and the vertical axis is altitude in $\mathrm{km}$ (from Kyrölä et al., 2006).

at night, while SBUV data are day-side. At low altitudes (15-25 km) near the equator, GOMOS finds less ozone than F-K (up to $20 \%$ less). The GOMOS results are not so reliable below $18 \mathrm{~km}$, but the discrepancy in the region $18-23 \mathrm{~km}$ is probably real, with an unclear reason up to now. Finally, there is a conspicuous deficit in both polar regions, very much more prominent in the South. This is the signature of the ozone hole, greater in 2003 than the earlier times of F-K data. However, the time evolution of this relative difference GOMOS versus F-K indicates that the deficit appears already in March, and grows during the South polar night (Kyrölä et al., 2006). Both deficits are probably linked to an increase of $\mathrm{NO}_{2}$ in the polar winters of 2003, w.r.t. the period around 1985 pertinent to the F-K climatology.

A more recent analysis provides ozone time series analysis for the period covering the six years period 2003-2008 (Kyrölä et al., 2010b). The average of ozone vertical profiles are displayed on Fig. 26 for various latitude bins. 

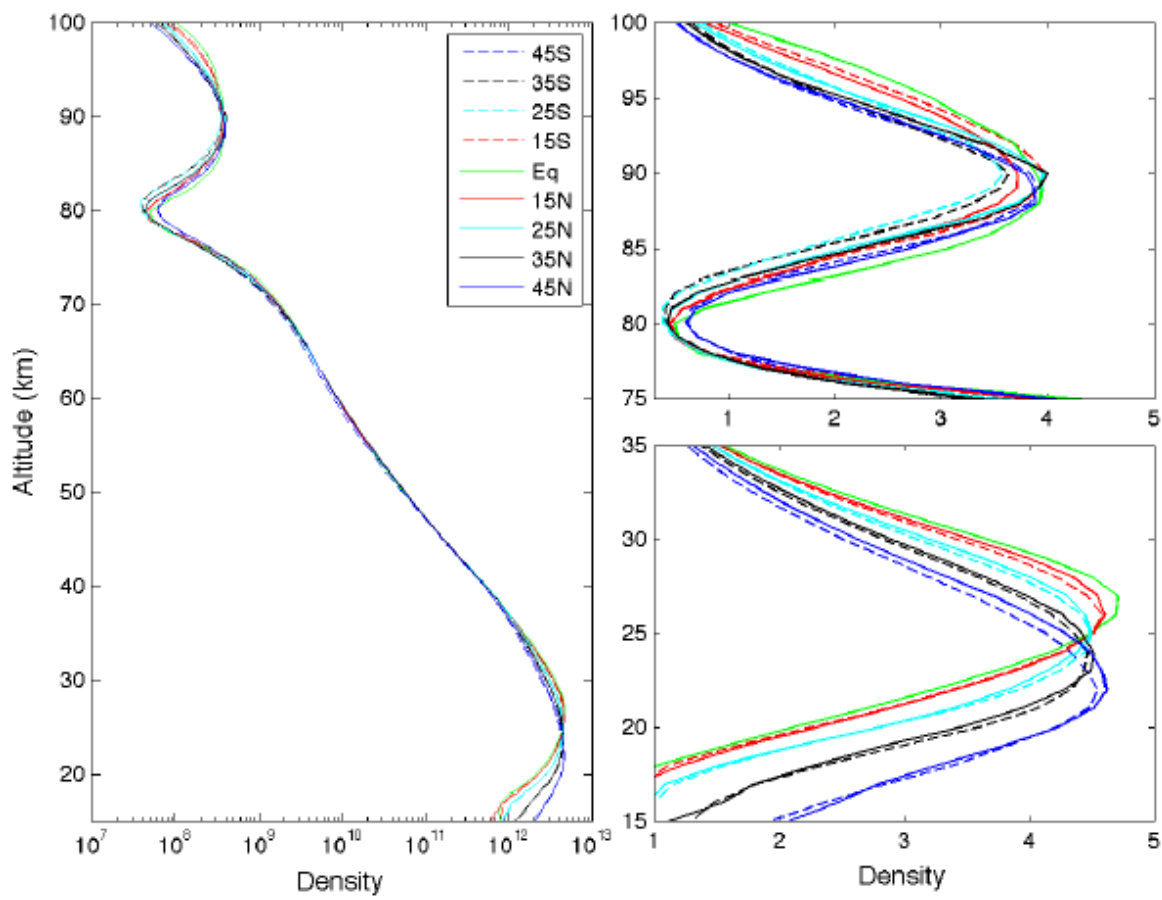

Fig. 26. Average vertical profiles of ozone in the period 2003-2008. The left panel shows vertical profiles at different latitudes (in $\mathrm{cm}^{-3}$ ). The upper right panel shows the same profiles in the mesosphere (in $10^{8} \mathrm{~cm}^{-3}$ ) and the lower right panel in the stratosphere (in $10^{12} \mathrm{~cm}^{-3}$ ) (from Kyrölä et al., 2010b).
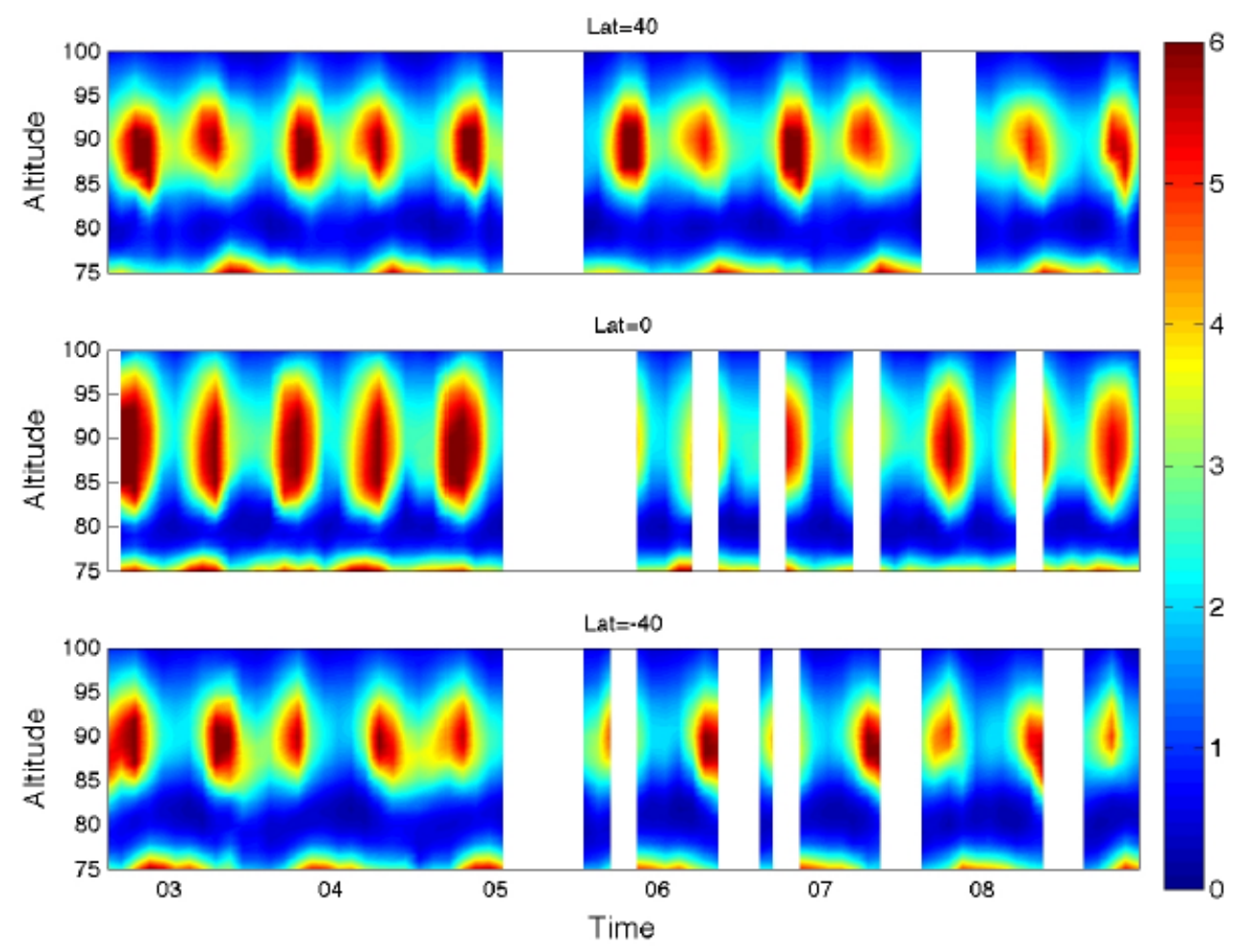

Fig. 27. Ozone number density (scaled by $10^{8} \mathrm{~cm}^{-3}$ ) in the MLT at three latitude belts in 1 August 2002-31 December 2008. Top: $30^{\circ} \mathrm{N}-$ $50^{\circ} \mathrm{N}$, Middle: $10^{\circ} \mathrm{S}-10^{\circ} \mathrm{N}$, Bottom: $30^{\circ} \mathrm{S}-50^{\circ} \mathrm{S}$ (from Kyrölä et al., 2010b). 
The ozone density in the stratosphere has a (well-known) seasonal behavior, with a maximum appearing during winterspring months. The ozone column in the range $15-100 \mathrm{~km}$ is calculated to be 244 Dobson at the equator, 264 Dobson in south mid-latitudes and 271 Dobson in North mid-latitudes for the period 2003-2008. There are two possible reasons for more ozone in the North mid-latitudes than in the South. We believe that the main reason is the greater activity of planetary waves in the Northern Hemisphere (due to the contrast between oceans and continents), leading to a faster Brewer-Dobson circulation and enhanced transport of ozone from equatorial region to mid- and high-latitudes (see for instance Tie et al., 1999). The second reason is the more severe anthropogenic depletion in the Southern Hemisphere $(-5.5 \%)$ than in the North $(-3 \%)$, as a result of air masses migrating from polar vortex toward lower latitudes in spring (Fioletov and Shepherd, 2005; WMO, Scientific Assessment of Ozone Depletion, 2007); this accounts for a 6.7 Dobson difference between North and South mid-latitudes, that is most of the GOMOS measured 7 Dobson difference. There would be no need for asymmetric transport. However, there is some ozone below $15 \mathrm{~km}$, whose column might be larger in the North than in the South, not included in the GOMOS $15-100 \mathrm{~km}$ columns.

\subsection{Ozone climatology in the Mesosphere-Lower Thermosphere (MLT)}

The night-time occultations of hot stars, whose spectra are UV-rich (highest cross section of ozone), enables measurement of ozone in the MLT with unprecedented refinement and coverage. In the MLT (Fig. 27) ozone exhibits a bi-annual oscillation (Kyrölä et al., 2010b), with maxima at equinoxes and minima at solstices. This behavior has been explained by Garcia and Solomon (1985) by invoking the effect of enhancement of gravity waves breaking at both solstices. They transport more $\mathrm{H}_{2} \mathrm{O}$ from below, a source of $\mathrm{H}, \mathrm{OH}$ and $\mathrm{HO}_{2}$ radicals that initiate a catalytic cycle of ozone destruction in this region. It may be noted that at $40^{\circ} \mathrm{N}$, the summer maximum lower boundary of the mesospheric ozone layer (at $85-87 \mathrm{~km}$ ) is lower by 2$3 \mathrm{~km}$ than the winter maximum lower boundary. This might be related to vertical eddy diffusion in this region, which has been shown through modeling to have a strong influence on the detailed vertical distribution of ozone (Smith, 2004).

\subsection{The tertiary ozone maximum}

The vertical profile of ozone mixing ratio usually shows two pronounced maxima (Fig. 28), one in the stratosphere around $\sim 25-30 \mathrm{~km}$ and the other in the mesosphere around $\sim 90 \mathrm{~km}$, corresponding to the two regions of production. A third ozone peak (mixing ratio) was reported first by Marsh et al. (2001), observed at $72 \mathrm{~km}$ altitude near the polar night terminator.

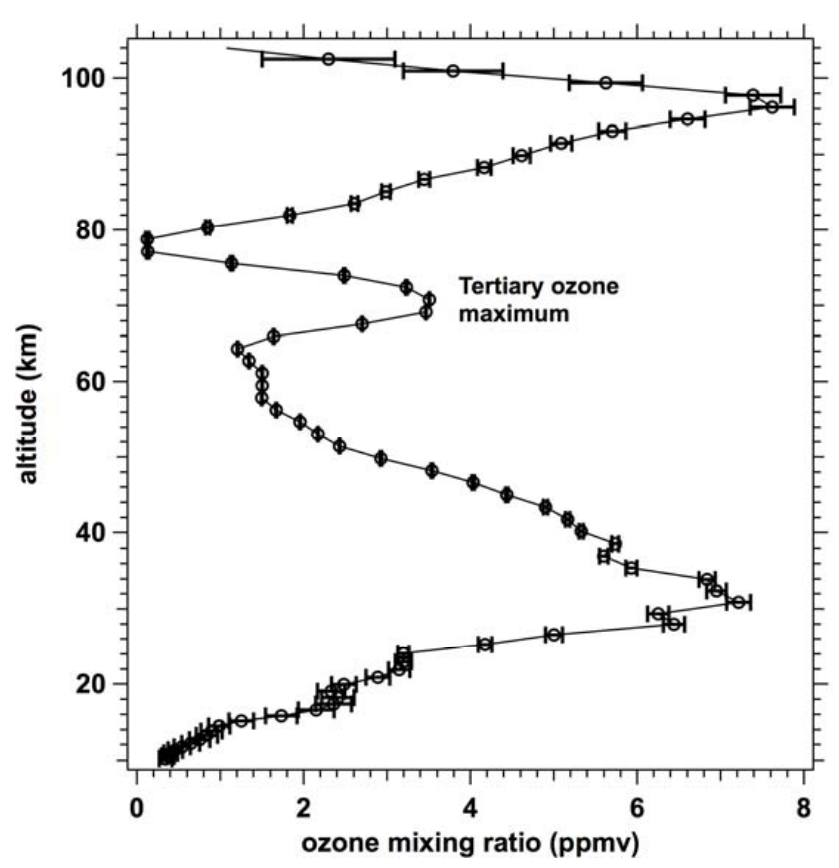

Fig. 28. Vertical profile of ozone mixing ratio measured by GOMOS with star S001 on orbit 4638 , at $71^{\circ} \mathrm{N}$ latitude on 30 January 2003. It displays the Tertiary Ozone Maximum, peaking at 3.6 ppmv on this particular profile. The air density is found in the Level 2 GOMOS product, derived from ECMWF and the MSISE 90 model.

In the mesosphere, ozone is destroyed by catalytic reactions involving odd hydrogen $\left(\mathrm{HO}_{\mathrm{x}}=\mathrm{H}+\mathrm{OH}+\mathrm{HO}_{2}\right)$, as discovered by Bates and Nicolet (1950). As a result, ozone is almost fully absent at an altitude of $\sim 80 \mathrm{~km}$. The tertiary ozone maximum (TOM) is explained by low concentrations of odd-hydrogen and the subsequent decrease in the destruction rate of $\mathrm{O}_{3}$, as modeled by Marsh et al. (2001). The reason is the decrease of $\mathrm{H}_{2} \mathrm{O}$ photolysis by a lack of solar UV radiation at high latitudes in winter. However, the predicted peak amplitude is $\sim 7 \mathrm{ppmv}$, while MLS and CRISTA observations were rather showing $\sim 3$ ppmv. It was suggested that the observed peak was underestimated by the instrumental FOV extent (Marsh et al., 2001).

GOMOS measurements, with their very good vertical resolution, are not affected by this problem (Fig. 28). Sofieva et al. $(2004,2009)$ have reported the analysis of 4 years of GOMOS data in the night (131500 occultations), yielding the first spatial-temporal distributions of tertiary ozone maxima (Fig. 29). This experimental TOM distribution was compared with that simulated by the WACCM model (The Whole Atmosphere Community Climate Model) developed under the leadership of NCAR. WACCM is a global model that extends from the ground to $4.5 \times 10^{-6} \mathrm{hPa}$, approximately $145 \mathrm{~km}$. 

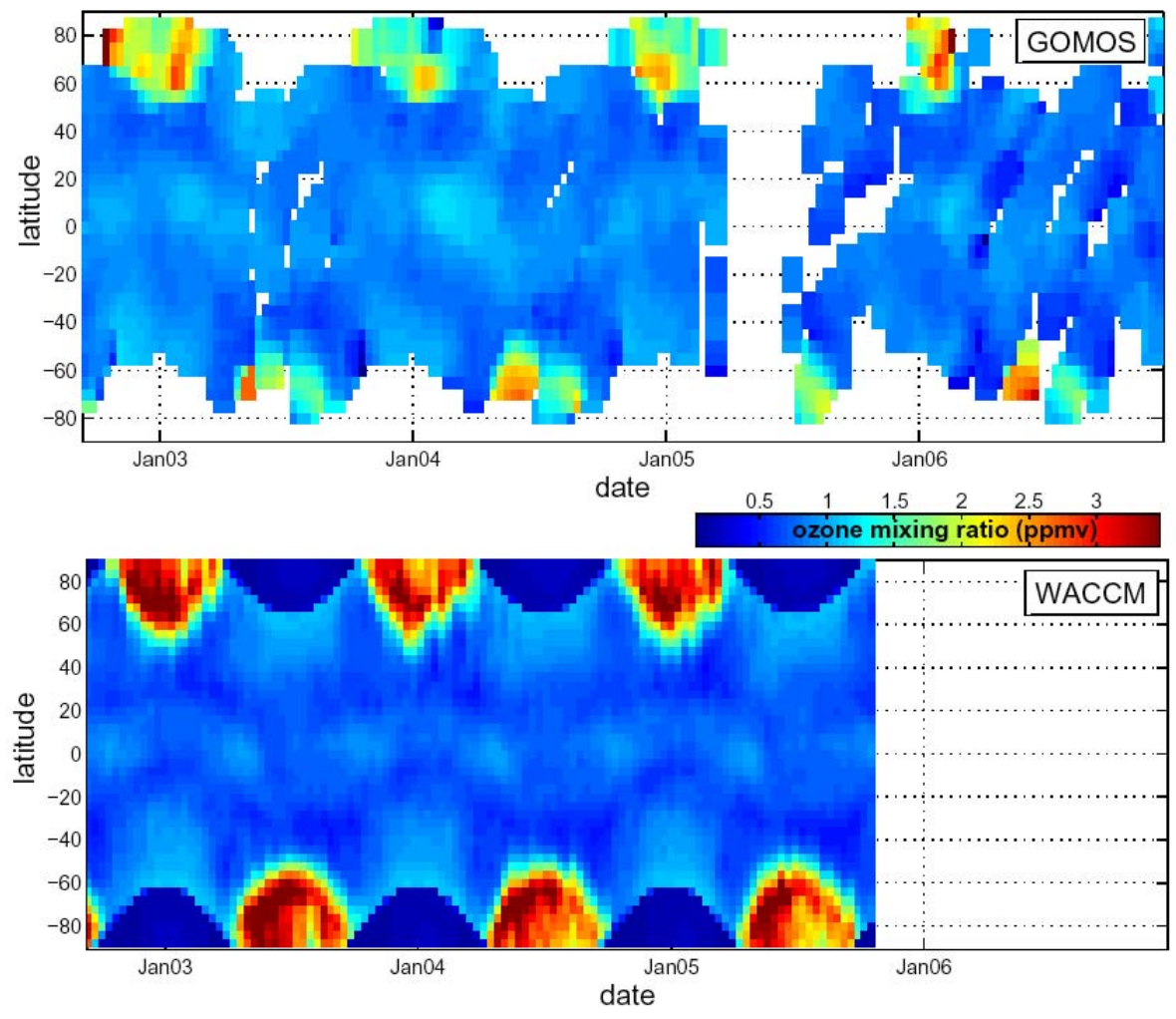

Fig. 29. Ozone mixing ratio at $72 \mathrm{~km}$. Top: from GOMOS measurements (zonal mean), bottom: from WACCM simulations (mean over three ensemble simulations for $0^{\circ}$ longitude, midnight). Temporal and latitudinal averaging is nearly the same for both experimental and model data (from Sofieva et al., 2009b).

We may summarize the findings of Sofieva et al. (2009b) on Tertiary Ozone Maximum as follows:

- The modeling of Hartogh et al. (2004) predicting large ozone values around the pole at upper altitudes (down to $75 \mathrm{~km}$ ), as a result of strong downward transport of atomic oxygen from the thermosphere, is not confirmed by the GOMOS data.

- The observed tertiary ozone maximum is not clearly isolated from the high latitudes, which is at variance with the model prediction in Hartogh et al. (2004).

- The overall evolution of the latitudinal distribution of ozone mixing ratio at $\sim 72 \mathrm{~km}$ (Fig. 29 , bottom) is well reproduced by the WACCM model. The only difference is that WACCM predicts slightly larger ozone mixing ratios (up to $5 \mathrm{ppmv}$ ) than those observed by GOMOS (not exceeding 4 ppmv, Fig. 28).

- The TOM is sometimes fully destroyed by two types of events: by sudden stratospheric warmings, followed by a strong downward transport of upper mesospheric air rich in $\mathrm{NO}$, another scavenger of ozone (through $\mathrm{NO}_{2}$ ), as discussed, for example, by Hauchecorne et al. (2007) for the January 2004 event, and by energetic particles precipitation such as Solar Proton events (SPE), as discussed in Seppälä et al. (2006) for the January 2005 SPE event.

\subsection{Ozone depletion during solar proton events}

Coronal mass ejections from the Sun can emit large amounts of high-energy protons into space, sometimes toward the Earth. Guided by the Earth's magnetic field, the protons can penetrate into the middle atmosphere in the polar regions and cause changes in the neutral composition. For example, following the ionization due to proton precipitation, substantial amounts of odd hydrogen and odd nitrogen $\mathrm{NO}_{\mathrm{x}}$ are produced which can then decrease the amount of ozone by tens of percent through catalytic reaction cycles. Increase in $\mathrm{HO}_{\mathrm{x}}$ decreases ozone in the mesosphere typically during the solar proton event (SPE) only, while $\mathrm{NO}_{\mathrm{x}}$ can have more lasting effects in the upper stratosphere due to its long chemical lifetime. Although infrequent, SPEs provide great possibilities to test our understanding of ozone chemistry of the middle atmosphere. Before the launch of GOMOS, the atmospheric effects of SPEs had already been observed by rocket and satellite instruments 

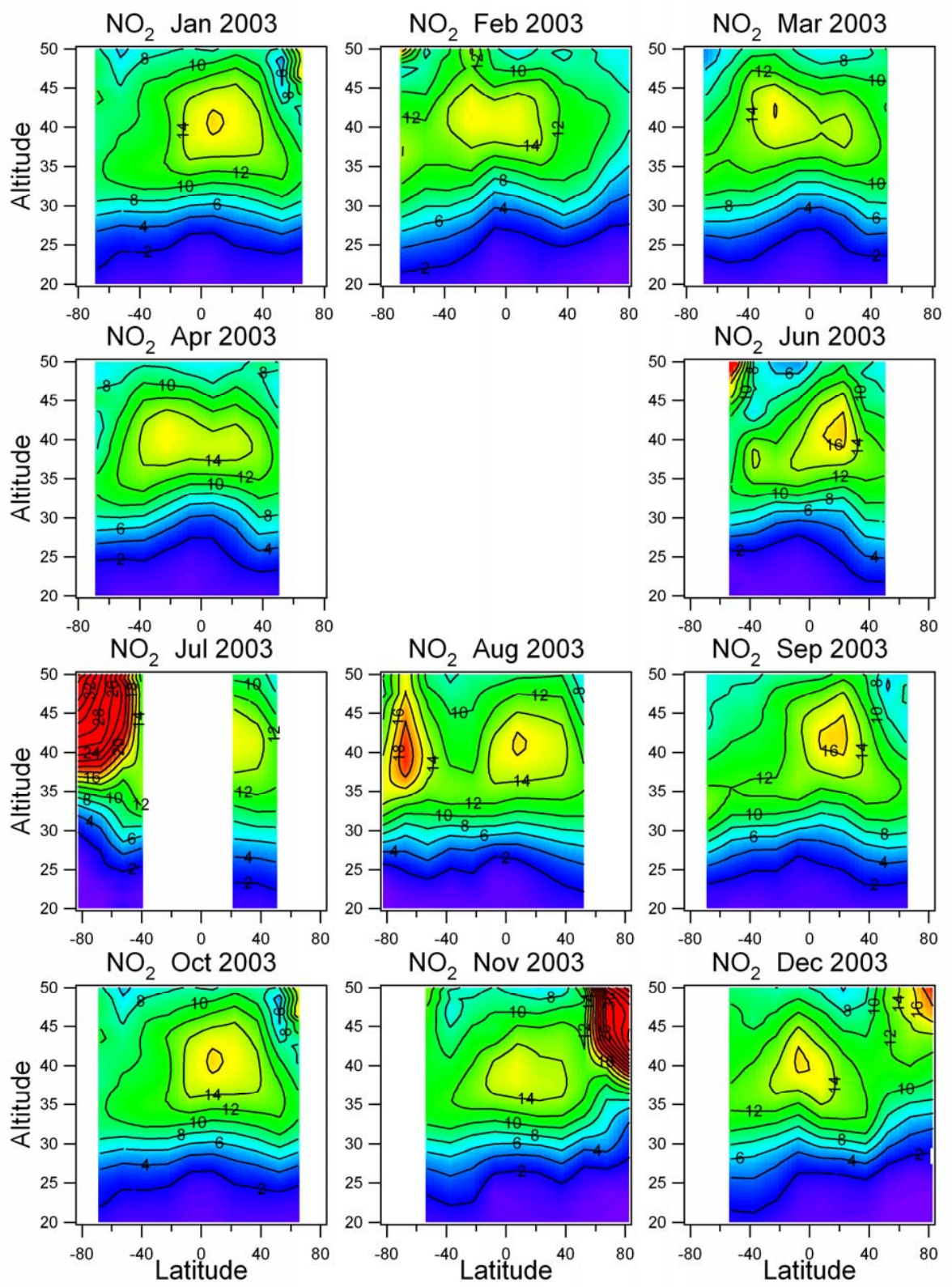

Fig. 30. Latitude-altitude maps of $\mathrm{NO}_{2}$ mixing ratio in ppbv for each month in 2003. A malfunction of the pointing mechanism occurred in May 2003. There are strong increases of $\mathrm{NO}_{2}$ in July 2003 in the south polar region, and in November 2003 in the north polar region, caused by the descent of air carrying NO molecules in the polar night (from Hauchecorne et al., 2005a).

since 1970s. However, these observations were typically limited in time and space such that, e.g., the data covered sunlit atmosphere only. Thanks to its unique night-time capabilities, GOMOS was the first satellite instrument to provide measurements of SPE effects on $\mathrm{NO}_{\mathrm{x}}$ and ozone in the winter polar regions. For example, Seppälä et al. (2004) and Verronen et al. (2005b) studied the SPE of October 2003, both the short-term and long-term effects. Shortterm ozone depletion larger than $60 \%$ was observed in the mesosphere $(65-80 \mathrm{~km})$ while a decrease of $\sim 40 \%$ lasting several months was seen in the upper stratosphere, and these were attributed to the substantial increases of $\mathrm{HO}_{\mathrm{x}}$ and $\mathrm{NO}_{\mathrm{x}}$, respectively. In a study of the January 2005 SPE, GOMOS observations of ozone, together with $\mathrm{OH}$ observations from the MLS/Aura instrument, were used in confirming the theoretically expected connection between mesospheric $\mathrm{HO}_{\mathrm{x}}$ increase and ozone depletion (Verronen et al., 2006). It was found that an order-of-magnitude increase observed in the $\mathrm{OH}$ concentration led to short-term ozone depletion by $90 \%$ at $70-80 \mathrm{~km}$ altitude. These results were 
found to be in good agreement with the predictions from the Sodankylä Ion and Neutral Chemistry model. Overall, GOMOS observations have advanced our understanding of SPE effects considerably, and have been especially important in studies of the winter polar regions.

\subsection{Climatology of $\mathrm{NO}_{2}$ and special events of $\mathrm{NO}_{2}$ enhancements in the polar night}

Since $\mathrm{NO}_{2}$ is a catalytic scavenger of ozone, it is important to monitor it at the same time. The main source of $\mathrm{NO}_{2}$ is the oxidation of $\mathrm{N}_{2} \mathrm{O}$ by atomic oxygen. Other significant potential sources are the upward transport of $\mathrm{NO}_{2}$ produced by lightning in the tropical upper troposphere and the downward transport of NO from the thermosphere or the mesosphere in the winter polar vortex.

Partitioning of odd nitrogen family, defined as $\mathrm{NO}+$ $\mathrm{NO}_{2}+\mathrm{NO}_{3}+\mathrm{N}_{2} \mathrm{O}_{5}$, is controlled in the stratosphere by the fast photochemical equilibrium between $\mathrm{NO}$ and $\mathrm{NO}_{2}$ and the slow photo-dissociation of $\mathrm{N}_{2} \mathrm{O}_{5}$ during daytime and the slow conversion of $\mathrm{NO}_{2}$ into $\mathrm{NO}_{3}$ and then into $\mathrm{N}_{2} \mathrm{O}_{5}$ during nighttime. Owing to these photochemical reactions, the $\mathrm{NO}_{2}$ concentration is never constant. It is slowly decreasing by night and increasing by day and rapidly increasing at sunset and decreasing at sunrise. It is therefore very important to take into account the local time when comparing measurements made by different instruments. In the case of GOMOS, nighttime observations are made during the ascending path of Envisat and the local time is approximately equal to the local hour of the ascending node, 22:00 LT.

Hauchecorne et al. (2005) have established a first climatology of night time $\mathrm{NO}_{2}$ from GOMOS occultations, represented on Fig. 30 in the form of monthly maps of mixing ratios in latitude-altitude (zonal average).

At mid- and low-latitudes, a maximum in the vertical profile is observed around $40 \mathrm{~km}$ in both hemispheres. The peak value ranges from 14 to $16 \mathrm{ppbv}$, depending on the month, and its latitude location varies between $20^{\circ} \mathrm{S}$ and $20^{\circ} \mathrm{N}$ with a shift toward the summer/autumn hemisphere, to the south from February to April and to the north from August to November. This value is slightly smaller than the results of Reburn et al. (1996) who found a $17 \mathrm{ppbv}$ peak of night-time $\mathrm{NO}_{2}$ around $3 \mathrm{hPa}(\sim 40 \mathrm{~km})$ in ISAMS and LIMS (limb infra red emission) January data at equatorial and south tropical latitudes. At high latitude, lower values are observed in general, except when a strong descent of air enriched in $\mathrm{NO}_{\mathrm{x}}\left(\mathrm{NO}+\mathrm{NO}_{2}\right)$ of mesospheric or thermospheric origin occurs inside the winter polar vortex.

It is interesting to note the strong peak of $\mathrm{NO}_{2}$ above $38 \mathrm{~km}$, with up to $38 \mathrm{ppbv}$ at $50 \mathrm{~km}$, in the northern high latitude upper stratosphere in November. This peak has been attributed by Seppälä et al. (2004) and Hauchecorne et al. (2006) to the formation of a large amount of $\mathrm{NO}_{\mathrm{x}}$ during the very intense solar proton event at the end of
October 2003. Such $\mathrm{NO}_{2}$ enhancement after strong solar proton events have been observed and simulated in the past (Jackman et al., 2000, 2001; Verronen et al., 2002). A very high mixing ratio (up to $36 \mathrm{ppbv}$ at $50 \mathrm{~km}$ ) is also detected in July at southern high latitude. In this case it is probably due to the strong diabatic descent of mesospheric and thermospheric air with high $\mathrm{NO}_{\mathrm{x}}$ mixing ratio in the southern winter polar vortex.

Indeed another $\mathrm{NO}_{2}$ descent event in 2004 was clearly not associated with particle precipitation, but rather was due to a dynamical cause (Hauchecorne et al., 2007). A layer of strongly enhanced $\mathrm{NO}_{2}$ was detected by GOMOS/ENVISAT starting on 15 January 2004 at $65 \mathrm{~km}$ in the north polar stratosphere with mixing ratios up to $1000 \mathrm{ppbv}$ (Fig. 31). The descent of the layer can be followed until 10 March down to $45 \mathrm{~km}$. The simultaneous destruction of $\mathrm{O}_{3}$ in the layer is clearly detected. An intense warming was observed in the north polar middle mesosphere at the time of formation of the $\mathrm{NO}_{2}$ layer by SABER/TIMED. This warming is an indication of a strong air descent in the polar vortex, bringing a large quantity of NO from the lower thermosphere. When such a descent occurs in the polar night, NO cannot be photodissociated before reaching an altitude where it is stable and can be transformed into $\mathrm{NO}_{2}$ by reaction with $\mathrm{O}_{3}$ (at $\sim 60$ $65 \mathrm{~km}$ ). Large enhancements of $\mathrm{NO}_{2}$ in the polar winter upper stratosphere/lower mesosphere have been observed in the past. They were attributed to the production of NO by large energetic particle precipitations (solar proton or energetic electron precipitations). These events are relatively rare. In the present 2004 case no significant particle precipitation was reported and we attribute the event to a pure atmospheric dynamical phenomenon: the breaking of atmospheric waves in the upper mesosphere, inducing a strong descent of thermospheric NO into the polar vortex. If the proposed mechanism appears to be frequent, it will be necessary to re-evaluate the contribution of stratospheremesosphere-lower thermosphere dynamical coupling in the polar stratospheric ozone budget.

Further $\mathrm{NO}_{2}$ and $\mathrm{NO}_{3}$ results for the years 2002 to 2008 are discussed in Kyrölä et al. (2010b).

\subsection{The influence of the QBO on tropical stratospheric ozone, $\mathrm{NO}_{2}$ and $\mathrm{NO}_{3}$}

In a companion paper of this GOMOS ACP issue, Hauchecorne et al. (2010) have investigated the impact of the Quasi-Biennial Oscillation (QBO) of the equatorial wind on the inter-annual variations of ozone and $\mathrm{NO}_{2}$. A six years period of GOMOS data from August 2002 to December 2008 recorded between $15^{\circ} \mathrm{S}$ and $15^{\circ} \mathrm{N}$ was analysed, first by establishing a seasonal mean, and then dividing all data by this seasonal mean. It is found that the $\mathrm{QBO}$ of the equatorial wind induces variations in the local concentration larger than $10 \%$ for $\mathrm{O}_{3}$ and larger than $25 \%$ for $\mathrm{NO}_{2}$. The $\mathrm{NO}_{2}$ and QBO signals are out of phase below $36 \mathrm{~km}$ and in phase 

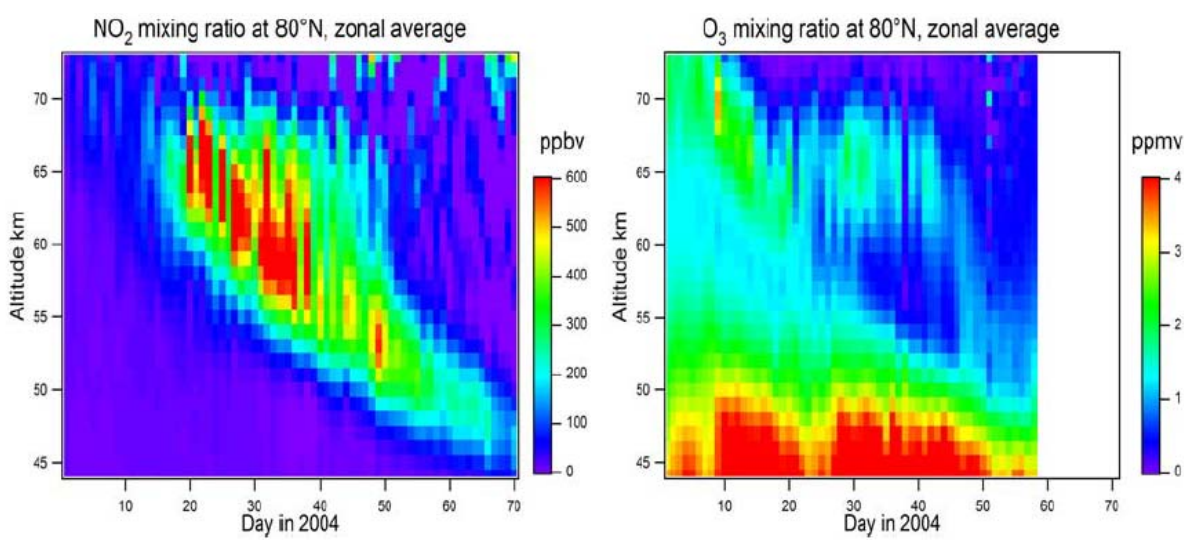

Fig. 31. Evolution of zonal averaged mixing ratio of $\mathrm{NO}_{2}$ (left) and $\mathrm{O}_{3}$ (right) at $80^{\circ} \mathrm{N}$ from GOMOS data between 1 January and $10 \mathrm{March}$ 2004 (from Hauchecorne et al., 2007).

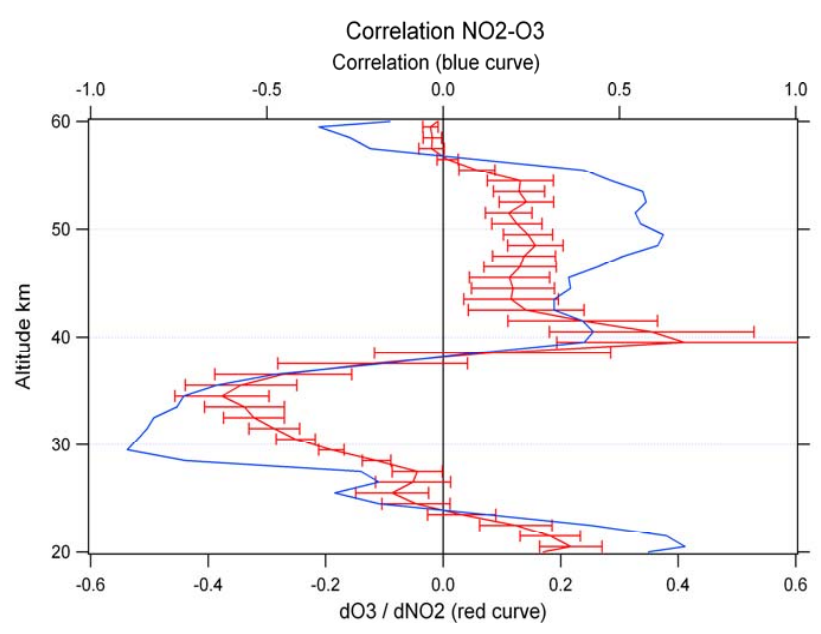

Fig. 32. Correlation between the deviation of $\mathrm{NO}_{2}$ and $\mathrm{O}_{3}$ from their mean seasonal values at the same altitude (blue curve) and relative variation of $\mathrm{O}_{3}$ per unit relative variation of $\mathrm{NO}_{2}$ (red curve) (from Hauchecorne et al., 2010).

above $36 \mathrm{~km}$. The correlation between the excess of $\mathrm{NO}_{2}$ and those of $\mathrm{O}_{3}, d \mathrm{O}_{3} / d \mathrm{NO}_{2}$, is displayed in Fig. 32. It has a strong vertical structure. Below $28 \mathrm{~km}$, the correlation is the sign of dynamical control of these species, while above $28 \mathrm{~km}$, there is an anti-correlation that is due to chemical control, through the catalytic destruction of $\mathrm{O}_{3}$ by $\mathrm{NO}_{\mathrm{x}}$. This transition was already observed in the data of SAGE II and explained as described above by comparison with models by Chipperfield et al. (1994) and Tian et al. (2007). The GOMOS results (Fig. 32) show a new transition at $38 \mathrm{~km}$, previously undetected: in the range $38-50 \mathrm{~km}$, a different regime is found with $\mathrm{O}_{3}$ and $\mathrm{NO}_{2}$ correlated with each other and anti-correlated with temperature, for which a new explanation is now proposed (Hauchecorne et al., 2010): an enhancement of the ascending motion (during one phase of

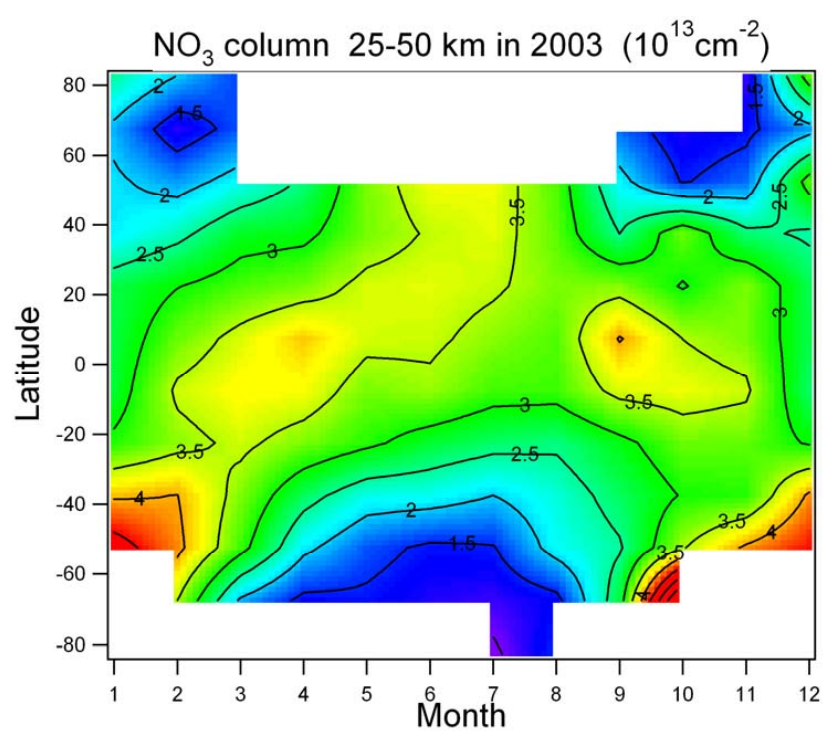

Fig. 33. Time-latitude cross section of $\mathrm{NO}_{3}$ stratospheric column $(25-50 \mathrm{~km})$ in $10^{13} \mathrm{~cm}^{-2}$ in 2003 , derived from GOMOS vertical profiles (from Hauchecorne et al., 2005).

the QBO) will cool adiabatically the atmosphere, and will increase ozone because the destruction of $\mathrm{O}_{3}$ by $\mathrm{O}_{3}+\mathrm{O} \rightarrow 2$ $\mathrm{O}_{2}$ reaction is strongly temperature dependent. It will not bring more $\mathrm{NO}_{2}$, because the maximum mixing ratio of $\mathrm{NO}_{2}$ is around $40 \mathrm{~km}$. Rather, this air ascent will increase the amount of $\mathrm{N}_{2} \mathrm{O}$ concentration available for $\mathrm{NO}_{\mathrm{x}}$ formation. The oxidation of $\mathrm{N}_{2} \mathrm{O}$ (coming from the troposphere) is the main source of $\mathrm{NO}_{\mathrm{x}}$ in this altitude region. Furthermore, because the destruction of $\mathrm{O}_{3}$ by $\mathrm{NO}_{2}$ decreases with temperature (see Reaction R1 in the next section), both species would increase with decreasing temperature, but this is a minor effect in the overall budget of $\mathrm{O}_{3}$ and $\mathrm{NO}_{2}$ in this region. 


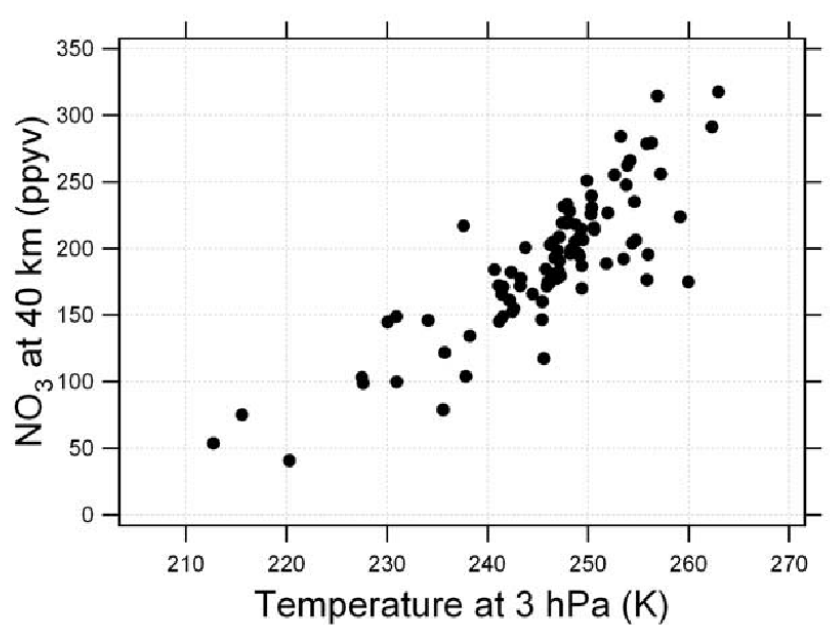

Fig. 34. $\mathrm{NO}_{3}$ mixing ratio at $40 \mathrm{~km}$ versus European Centre for Medium-Range Weather Forecasts' (ECMWF) temperature at $3 \mathrm{hPa}(\sim 40 \mathrm{~km})$, a result of the temperature-dependent reaction rate producing $\mathrm{NO}_{3}$ (from Hauchecorne et al., 2005).

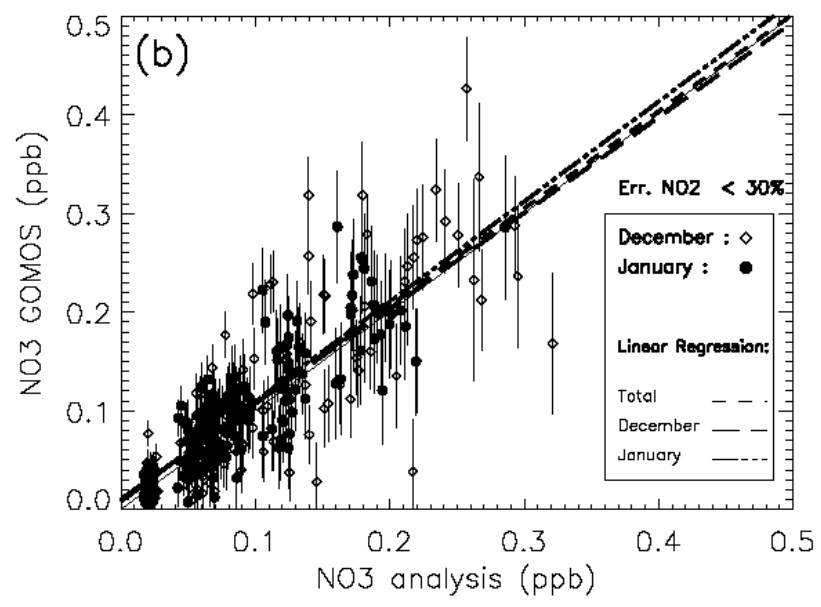

Fig. 35. GOMOS $\mathrm{NO}_{3}$ measurement as a function of analyzed $\mathrm{NO}_{3}$ for cases when $\mathrm{NO}_{2}$ error measurements are less than $30 \%$. $\mathrm{NO}_{3}$ error measurements are indicated with vertical lines. The linear regressions for the different periods are represented with dashed lines. The 1-to-1 line is represented with a thin black line. The two periods of GOMOS $\mathrm{O}_{3}$ and $\mathrm{NO}_{2}$ assimilated data are 5 to 6 December 2002, and 30 January 2003, with occultations of the star Beta Carinae (GOMOS star S029, visual magnitude 1.67, temperature $10200 \mathrm{~K}$ ) in a latitude band $0-1^{\circ} \mathrm{N}$ for the first period, and $16-17^{\circ} \mathrm{N}$ for the second period (from Marchand et al., 2004).

\subsection{Climatology of $\mathrm{NO}_{3}$ and temperature retrieval}

The measurement of the nitrate radical $\mathrm{NO}_{3}$ by the GOMOS instrument on board the ENVISAT platform is the first satellite measurement of this species (Fig. 14). $\mathrm{NO}_{3}$ exists only at night, being photo-dissociated very quickly. Therefore, it is not possible to measure it with solar occultations, and it has no strong infra-red emission.

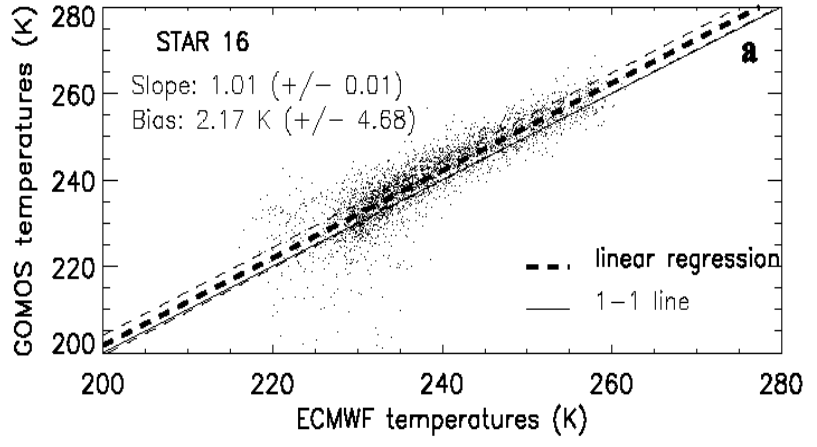

Fig. 36. Temperature retrieved from $\mathrm{NO}_{3}$ and $\mathrm{O}_{3}$ GOMOS data (star number 16, Antares Alpha Scorpio) as a function of the corresponding ECMWF temperature. The slope is $1.01 \pm 0.01$. GOMOS-retrieved temperatures are warmer by about $2 \mathrm{~K}$ than ECMWF temperatures. When more stars are included in the study, the bias is found to be $2.67 \mathrm{~K}$ (from Marchand et al., 2007).

A climatology of $\mathrm{NO}_{3}$ has been established for the first time from 2003 night-time GOMOS measurements (Hauchecorne et al., 2005), using the same occultations that produced a $\mathrm{NO}_{2}$ climatology. The chemistry of $\mathrm{NO}_{3}$ is rather simple, being associated to $\mathrm{O}_{3}, \mathrm{NO}_{2}$ and $\mathrm{N}_{2} \mathrm{O}_{5}$, with the following three reactions:

$$
\begin{aligned}
& \mathrm{NO}_{2}+\mathrm{O}_{3} \rightarrow \mathrm{NO}_{3}+\mathrm{O}_{2} \\
& \mathrm{NO}_{3}+\mathrm{NO}_{2}+\mathrm{M} \rightarrow \mathrm{N}_{2} \mathrm{O}_{5}+\mathrm{M} \\
& \mathrm{N}_{2} \mathrm{O}_{5}+\mathrm{M} \rightarrow \mathrm{NO}_{3}+\mathrm{NO}_{2}+\mathrm{M}
\end{aligned}
$$

$\mathrm{NO}_{3}$ is produced by reaction of $\mathrm{NO}_{2}$ with $\mathrm{O}_{3}$ (Reaction $\mathrm{R} 1$ ). This reaction, whose rate constant is strongly temperature dependent, affects the nighttime decay rate of $\mathrm{NO}_{2}$. The $\mathrm{NO}_{3}$ sink in the stratosphere is its reaction with $\mathrm{NO}_{2}$ (Reaction R2), which produces $\mathrm{N}_{2} \mathrm{O}_{5}$. Finally, the other source of $\mathrm{NO}_{3}$ is the thermal decomposition of $\mathrm{N}_{2} \mathrm{O}_{5}$ (Reaction R3). The $\mathrm{NO}_{3}$ concentrations are close to zero on the day-side.

The main features found for $\mathrm{NO}_{3}$ climatology are as follows: a peak mixing ratio of up to $320 \mathrm{pptv}$ is reached between 41 and $45 \mathrm{~km}$. At low latitude, a semiannual variation is observed in $\mathrm{NO}_{3}$ peak concentration and stratospheric column with a maximum at equinoxes (Fig. 33). At middle and high latitudes, the variation of $\mathrm{NO}_{3}$ is annual with a maximum in summer. The peak concentration is linked to the total column 25$50 \mathrm{~km}$, plotted in zonal average (latitude/season) on Fig. 33. The $\mathrm{NO}_{3}$ mixing ratio in the upper stratosphere is also correlated with temperature through the thermal dependence of Reaction (R1): $\mathrm{NO}_{2}+\mathrm{O}_{3} \rightarrow \mathrm{NO}_{3}+\mathrm{O}_{2}$, and consequently the maximum $\mathrm{NO}_{3}$ column follows more or less the latitude of the sub-solar point. 
Indeed, from their $\mathrm{NO}_{3}$ climatology GOMOS data Hauchecorne et al. (2005) have correlated the $\mathrm{NO}_{3}$ mixing ratio measured by GOMOS to the ECMWF temperature analysis at the point of occultation (Fig. 34). There is a clear dependence of $\mathrm{NO}_{3}$ on temperature.

In an interesting exercise, Marchand et al. (2004) have compared some GOMOS $\mathrm{NO}_{3}$ densities with those predicted by a chemistry model in which the GOMOS ozone and $\mathrm{NO}_{2}$ densities were assimilated. Restricting the data to the best $\mathrm{NO}_{2}$ measurements (less than $30 \%$ error, $20-45 \mathrm{~km}$ ) the plot of measured values versus model prediction may be fitted by a linear regression (Fig. 35) with a slope of $0.98 \pm 0.04$. The fact that linear regression slopes are so close to unity indicates that there is no substantial bias in GOMOS $\mathrm{NO}_{3}$ data. It also suggests that $\mathrm{O}_{3}, \mathrm{NO}_{2}$ and $\mathrm{NO}_{3}$ GOMOS measurements are self consistent chemically and that the nighttime $\mathrm{NO}_{3}$ chemistry is well understood.

In this exercise, Marchand et al. (2004) have assumed that the stratospheric temperature was the one given by ECMWF analysis. However, it is possible to build a reverse approach: the local temperature may be determined from the simultaneous measurements of $\mathrm{NO}_{2}, \mathrm{NO}_{3}$ and $\mathrm{O}_{3}$ from GOMOS by using the temperature dependence of reaction rates in (R1) and assuming steady state equilibrium.

This was done by Marchand et al. (2007) in an attempt, never demonstrated before, to determine the temperature from chemical measurements. Figure 36 indicates the GOMOS retrieved temperature for a number of occultations of the star Antares (GOMOS star number 16) as a function of ECMWF temperature. The GOMOS temperatures are warmer by about $2 \mathrm{~K}$. When increasing the sample to 4938 occultations of 4 bright stars (for which the temperature is retrieved with a precision better than $3.5 \mathrm{~K}$ ), between $50^{\circ} \mathrm{S}$ and $50^{\circ} \mathrm{N}$, and 30 to $40 \mathrm{~km}$ of altitude, the bias and dispersion are found to be $2.67 \mathrm{~K}$ and $3.01 \mathrm{~K}$, respectively.

What is the source of the bias? Assuming that ECMWF temperature represents the true temperature (i.e. no bias), Marchand et al. (2004) have determined how the rate constants of Reactions (R1) and (R2) should be modified from their current JPL recommended values, which are known for stratospheric temperatures with a $30 \%$ error bar. They found that the temperature bias could be eliminated by changing only the activation energy of Reaction (R1): instead of the JPL-recommended value $\mathrm{k} 1=1.2 \times 10^{-13}$ $\exp (-2450 / T)$, they propose a new temperature dependence: $\mathrm{k} 1==1.2 \times 10^{-13} \exp (-2400 / T)$, which changes k1 by only $23 \%$, within the uncertainty bar. This work is similar to the one of Renard et al. (2005), with somewhat different results. However, the work of Renard et al. (2005) relied on only a very limited number of balloon observations, while here the statistic sample is much larger.

It illustrates how the stratosphere itself may be used as a gigantic laboratory to determine fundamental reaction rates, provided that simultaneous measurements are performed under a known temperature and pressure environment.

\subsection{GOMOS observations of OCIO}

GOMOS has detected the presence of a global OClO layer in the upper stratosphere that had not been reported before (Fussen et al., 2006). Although $\mathrm{OClO}$ is not directly responsible for the ozone destruction, it plays a fundamental role in the inorganic chlorine (Cly) chemistry, responsible for the Cl-based catalytic destruction cycle of ozone, particularly active in the polar regions. It is indeed produced by a reaction involving $\mathrm{ClO}$ and $\mathrm{BrO}$, another ozone catalytic scavenger:

$\mathrm{ClO}+\mathrm{BrO} \rightarrow \mathrm{OClO}+\mathrm{Br}$

and therefore $\mathrm{OClO}$ is a proxy of ozone $\mathrm{Cl}-$ and $\mathrm{Br}$-based scavengers. The correct modeling of its abundance is a crucial test for our understanding of the stratospheric halogen chemistry. The major sink for $\mathrm{OClO}$ is its rapid photolysis by the solar radiation due to a large absorption cross section well characterized by a strong differential structure in the UV wavelength range. This feature has allowed the measurement of OClO, a species that is only present at very small concentrations.

Since the first detection of atmospheric $\mathrm{OClO}$ in Antarctica (Solomon et al., 1987), total column densities were retrieved from ground-based, airborne and satellite measurements. So far, the measurement of the vertical profile of the $\mathrm{OClO}$ number density has been performed in a very limited number of experiments, and only in polar regions, as, for example, the balloon experiments reported by Pommereau and Piquard (1994), and Renard et al. (1997a).

In this context, Fussen et al. (2006) have reported the first global determination of the stratospheric OClO distribution measured during the year 2003 by the stellar occultation spectrometer GOMOS. The analysis was restricted to about 4500 occultations of the five brightest stars in the range $335-390 \mathrm{~nm}$, where OClO absorption spectral features can be detected using the DOAS method, and distinguished from $\mathrm{NO}_{2}$ spectral features. Fussen et al. (2006) had to average the transmission spectra in latitude bins of $5^{\circ}$ (zonal and time average) in order to identify the small spectral features (below 0.01 differential transmittance). This was the first latitude-altitude map of OClO slant path densities, represented on Fig. 37, and was compared to the prediction of BASCOE model (Errera and Fonteyn, 2006).

The strong enhancements in both polar nights were known; they are reproduced by the model, with however, a much larger extension outside the polar vortex in the data of the north polar region. In addition, the data show the presence of a global OClO layer in the upper stratosphere that had not been reported before. For more details on the spectral detection of $\mathrm{OClO}$ with GOMOS, the reader is referred to Fussen et al. (2006).

Tétard et al. (2009) made a further study of $\mathrm{OClO}$ GOMOS data, correlated with simultaneous $\mathrm{O}_{3}$ and $\mathrm{NO}_{2}$ measurements, and during several winters of the Northern Polar regions. In January 2008, the center of the polar 

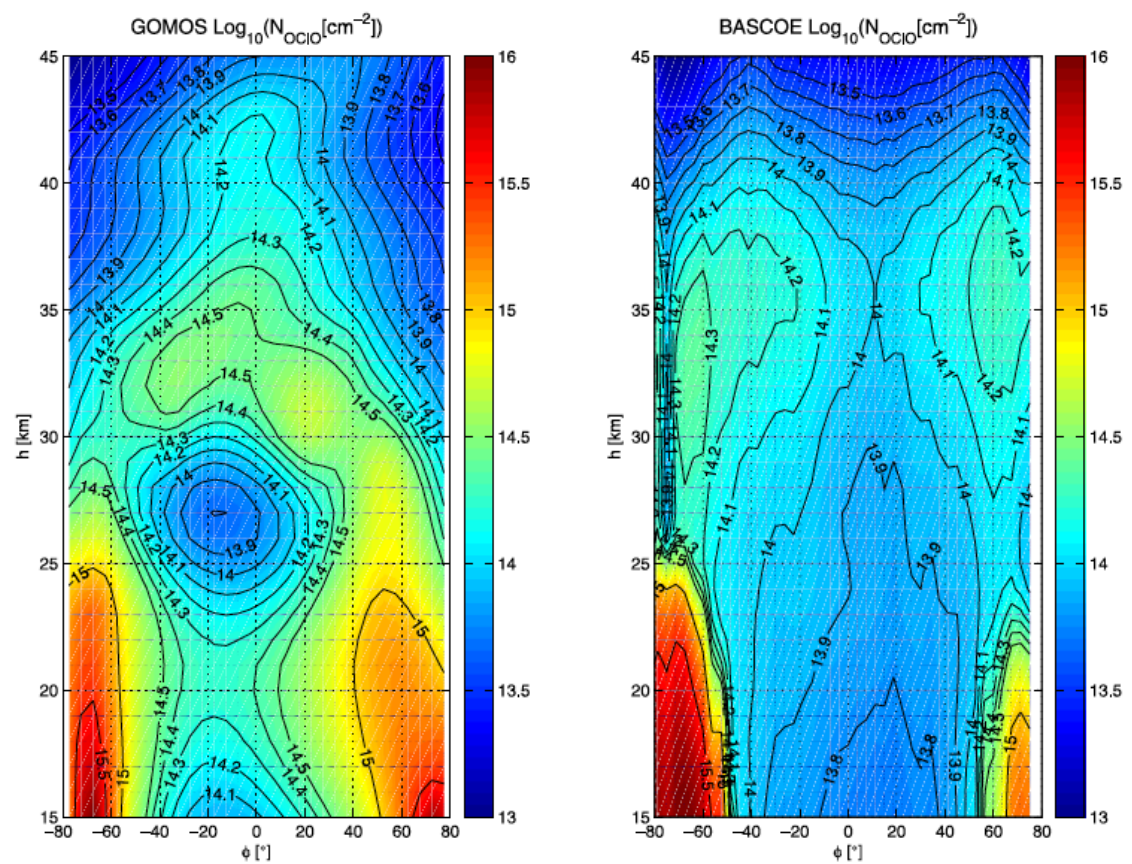

Fig. 37. Isocontours (latitude-altitude) of the OClO slant column densities measured by GOMOS and modeled by the BASCOE model at the same geolocation and time for the measurement periods (14 January-9 February 2003) and (20 July-30 December 2003). Both polar regions are probed during polar night (from Fussen et al., 2006).
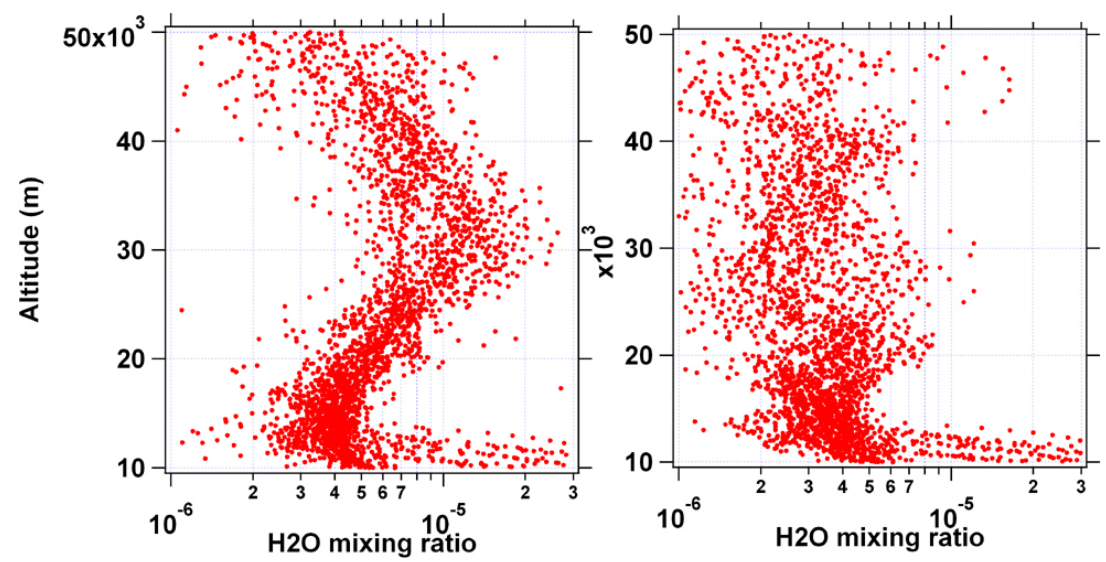

Fig. 38. Comparison of the $\mathrm{H}_{2} \mathrm{O}$ mixing ratios found with the official algorithm V6.0 (left) and the new Level 1B algorithm V7.0 (right). This series of occultations of star Canopus over one month covered latitudes from $23^{\circ} \mathrm{S}$ to $5^{\circ} \mathrm{S}$. The bump at $30 \mathrm{~km}$ has completely disappeared (right) with the new version of the algorithm (from Bertaux et al., 2009).

vortex was strongly offset from the pole. As a result, along a constant latitude circle of $72^{\circ}$, strong variations of these species were observed to be correlated (as a function of longitude) in the lower stratosphere. Inside the polar vortex, $\mathrm{NO}_{2}$ was weak, while $\mathrm{OClO}$ was strong, with a reversed situation outside of the polar vortex. This was a sign of $\mathrm{NO}_{2}$ trapping on PSC particles in the vortex (under the form of $\mathrm{HNO}_{3}$, denoxification) and halogen activation, since $\mathrm{OClO}$ is a good qualitative indicator of chlorine activation, and a good quantitative indicator of $\mathrm{ClO}$ presence (Sessler et al., 1995).

\subsection{Water vapor measurements at $936 \mathrm{~nm}$}

GOMOS is the first instrument to measure water vapour by the technique of stellar occultation. The status of GOMOS $\mathrm{H}_{2} \mathrm{O}$ analysis was reported recently by Bertaux et al. (2009). Nine stars are sufficiently bright to provide useful measurements for stratospheric $\mathrm{H}_{2} \mathrm{O}$, with a reasonable latitude/season coverage. Figure 16 shows a comparison of a modeled transmission with one actual measurement. Based on the $\mathrm{H}_{2} \mathrm{O}$ results obtained with version GOPR 6 of the software, Montoux (2008) has compared the $\mathrm{H}_{2} \mathrm{O}$ 


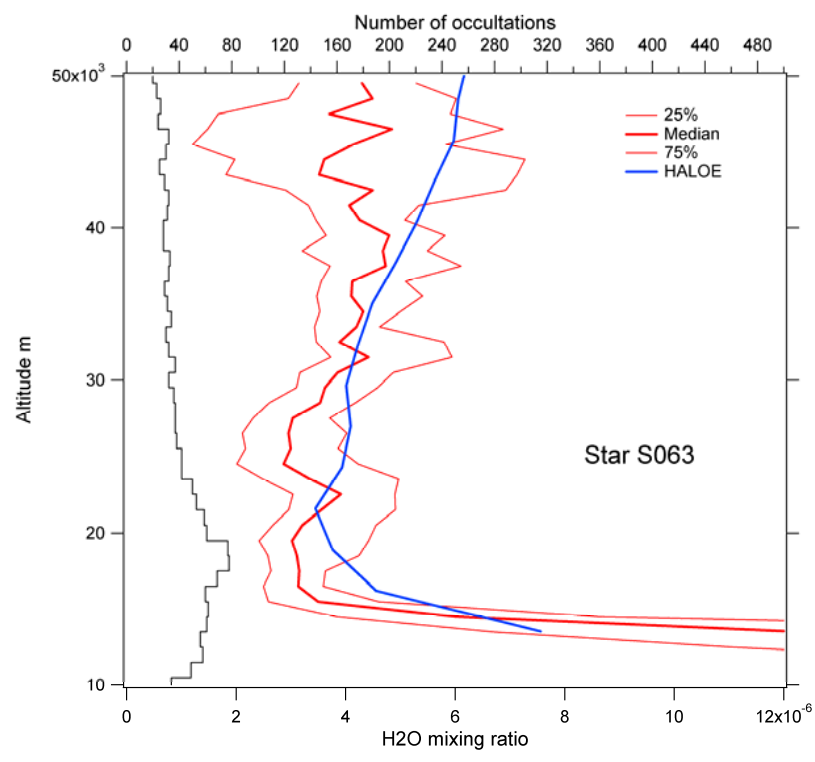

Fig. 39. Comparison of $\mathrm{H}_{2} \mathrm{O}$ vertical profiles obtained by HALOE (blue line) and GOMOS (red lines). In black and upper scale: the number of GOMOS measurements in each altitude bin obtained with the occultation of star Gamma Gruis. The thick red line is the median value, and the thin lines are the upper and lower quartile of the distribution in each bin (from Bertaux et al., 2009).

GOMOS average measurements with the IR solar occultation HALOE on UARS, and the microwave limb sensor MLS on AURA. In the upper troposphere, GOMOS is thought to be correct, slightly higher than MLS data, while HALOE shows a significant dry bias. In the stratosphere, neither HALOE nor MLS show the bump present in the GOMOS data around $30 \mathrm{~km}$ (Fig. 38, left), while all 3 sets of measurements are similar around $40 \mathrm{~km}$, at $\sim 5 \mathrm{ppmv}$ (oxidation of methane produces $\mathrm{H}_{2} \mathrm{O}$ at this altitude). This exercise of validation (in fact, invalidation in this case) led the GOMOS team to review in detail the $\mathrm{H}_{2} \mathrm{O}$ data processing. A problem was identified: the CCD dedicated to $926 \mathrm{~nm}$ radiation presents a severe pixel-to-pixel non-uniformity of the CCD detector, while the star image (smaller than one pixel) on the CCD is moving because of refraction/scintillation. The PRNU is about $\sim 20 \%$, while absorption features are of the order of a few $\%$ in the lower stratosphere. This was calibrated on ground, and in principle the Level 1b software takes care of the motion of the star on the particular pixels (at most, two pixels in the vertical direction). However, it was discovered in flight serendipitously that there is also a rather large subpixel non-uniformity. Some episodes of malfunction of the front steering mechanism occurring in 2008 could be used to reconstruct a much better PRNU including sub-pixel response.

A test with a new Level $1 b$ algorithm taking into account the sub-pixel response was performed on a limited set of occultations. Figure 38 compares the $\mathrm{H}_{2} \mathrm{O}$ densities found

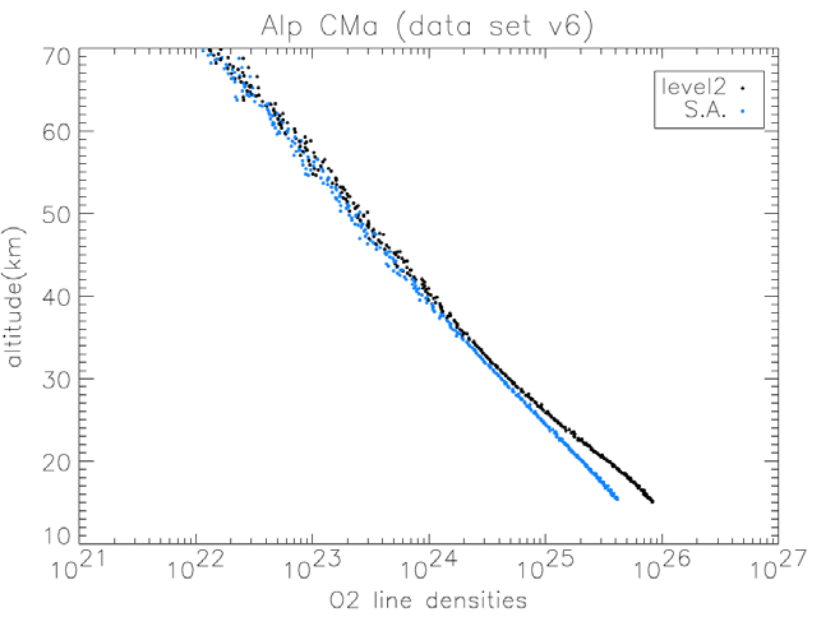

Fig. 40. Comparison of oxygen line densities retrieved from GOMOS $760 \mathrm{~nm} \mathrm{O}_{2}$ band transmission measurements for 6 occultations of the star Sirius (Alpha Canis Major). Black points: with models computed with HITRAN 1996 data base. Blue points: with HITRAN 2000 data base. There is an increasing difference with decreasing altitude.

with the nominal algorithm (left) and the new algorithm (right). The bump at $30 \mathrm{~km}$ has completely disappeared and reasonable values (3-6 ppmv) are retrieved in the lower stratosphere.

In Fig. 39 are compared the statistical distribution of GOMOS profiles (median, rather than average, to avoid the influence of bad data, and upper and lower quartiles) with HALOE average for similar latitude and season. GOMOS is drier than HALOE above $40 \mathrm{~km}$. However, the absorption features of $\mathrm{H}_{2} \mathrm{O}$ at these altitudes are very weak in the $936 \mathrm{~nm}$ band, and the GOMOS values are more uncertain above $40 \mathrm{~km}$.

These new results are very encouraging. When GOMOS data are reprocessed with this new algorithm and subpixel PRNU response, it will open the way to a better understanding of the $\mathrm{H}_{2} \mathrm{O}$ trend and possibly of the mechanisms of injection in the stratosphere, two subjects of controversy at this time.

\subsection{Measurements of $\mathrm{O}_{2}$}

Measurements of $\mathrm{O}_{2}$ are made in the $\mathrm{A}$ band of $\mathrm{O}_{2}$ around $760 \mathrm{~nm}$ (Fig. 15). The basis of the GOMOS retrieval is to compare the transmission spectra to a series of pre-calculated model spectra, computed with the HITRAN data base, and five typical US standard profiles. In the version GOPR 5 of the GOMOS software, we used the 1996 HITRAN data base, while in the version GOPR 6 (the present one) we used a later version, HITRAN 2000. In Fig. 40 the line densities for 6 occultations of Sirius obtained in 2003 are represented, retrieved for the two versions of the data base. There is a decrease of the $\mathrm{O}_{2}$ line density, especially large at the 


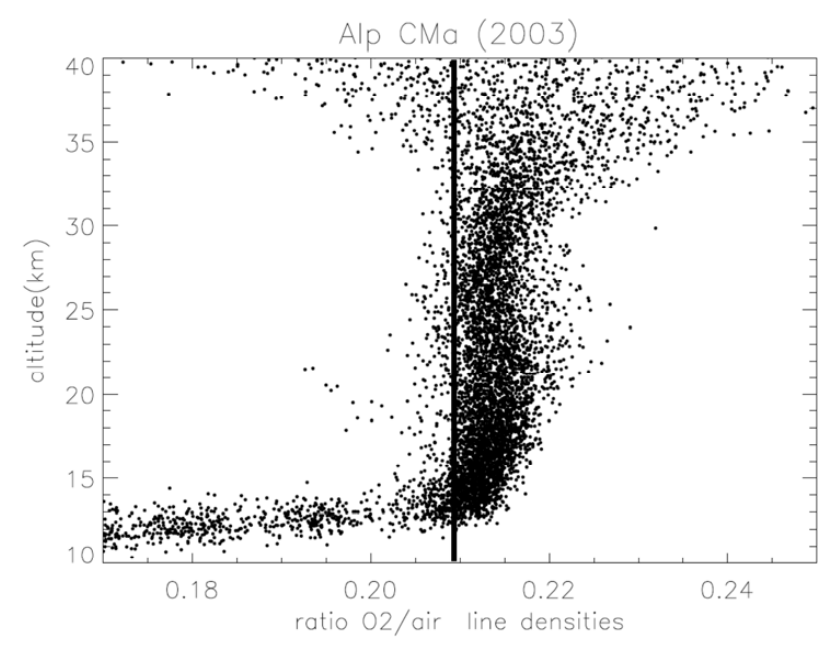

Fig. 41. A number of occultations of Sirius are considered during 2003. Each point is the ratio $N\left(\mathrm{O}_{2}\right) / N_{\text {air }}$ of the retrieved line density $N\left(\mathrm{O}_{2}\right)$ from GOMOS to the line density $N_{\text {air }}$ computed from ECMWF. A vertical bar is placed at the value 0.20946, the normal $\mathrm{O}_{2}$ mixing ratio. There is an unexplained bias where the measurements are better.

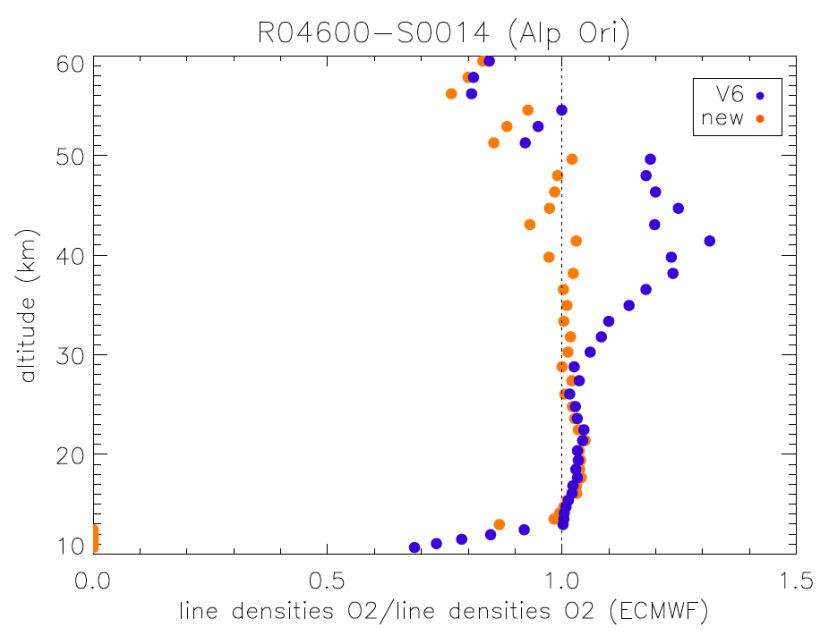

Fig. 42. Comparison of the retrieved line densities with the present version GOPR 6 of the algorithm, and a new version, where the modeled transmissions are adapted from ECMWF to the time and location of the occultation. This is one occultation of Alpha Orionis during orbit 4600 .

lowest altitude $(\sim-50 \%)$ with HITRAN 2000. In fact, when compared to ECMWF data, the GOPR 5 values were much too high. Spectroscopic data for $\mathrm{O}_{2}$ were wrong in HITRAN 1996.

The current Level 2 GOMOS product (GOPR 6) includes the retrieved line and local densities of $\mathrm{O}_{2}$, as well as the line and local densities of air delivered by ECMWF from analysis, for the time and location of the occultation. This gives us the opportunity to compare the GOMOS- retrieved quantities to a serious reference. In Fig. 41 are plotted, for several tens of Sirius occultations of 2003, the ratio $N\left(\mathrm{O}_{2}\right) / N_{\text {air }}$ of the retrieved line density $N\left(\mathrm{O}_{2}\right)$ from GOMOS to the line density $N_{\text {air }}$ contained in ECMWF (obtained by proper integration along the line of sight of the local density). In some sense, it is the mixing ratio of oxygen measured by GOMOS, when averaged along the line of sight. The value of the $\mathrm{O}_{2}$ mixing ratio in air is in principle $20.946 \%$, while we find much lower values below $15 \mathrm{~km}$, and a large dispersion above $35 \mathrm{~km}$; in the range $15-35 \mathrm{~km}$, the $1 \sigma$ dispersion of GOMOS measurements is about $\sim 1 \%$, while the bias is $+2 \%$. The study of all measurements (15$20 \mathrm{~km}$ ) on the 9 brightest stars over the year 2003, 2006 and 2008 showed us that the bias was variable with season and latitude, the pattern being somewhat similar from year to year (not shown here). The bias may go up to $6 \%$, and may be also negative. Therefore, it is difficult to invoke again an incorrect spectroscopic data base, which would likely give a bias always with the same sign at a constant altitude. This systematic difference with the ECMWF may be explained by three possibilities: (i) the ECMWF reference is biased itself; (ii) the retrieval algorithm of $\mathrm{O}_{2}$ line density (as described in Sect. 5.6) is incorrect or (iii) the choice of a small number of pre-calculated model transmissions (5 typical US standard profiles) is insufficient to represent all possible transmission spectra.

In order to test hypothesis (iii), a new software was developed at LATMOS. Instead of comparing all occultations to a limited number of typical US standard profiles, the table of modeled transmissions is computed for the very ECMWF profile that corresponds to the time and place of occultation. It is certainly a good approximation of reality, better than using the US standard profiles. However, this procedure is computer-time consuming, and only limited samples could be run up to now. Figure 42 shows an example of compared retrieved mixing ratios of line density profiles (with ECMWF being the reference) with the standard method (GOPR 6) and the new method. Both profiles are normalized to 1 for the mixing ratio $20.946 \%$. The bias of the new method is very similar below $28 \mathrm{~km}$ (also checked on 10 other occultations), but much lower above $28 \mathrm{~km}$ : this new method may improve the retrieval accuracy above $28 \mathrm{~km}$. Further studies are needed in this field. It is probably useful to perform a systematic comparison with ECMWF line density profiles (with both method, GOPR 6 and new); perhaps, the variations of bias as a function of altitude, latitude, longitude, season, may be then analyzed, to disentangle the respective contributions of the three possible sources of biases mentioned above.

\subsection{Aerosols and Polar Stratospheric Clouds (PSCs)}

At the time of the ENVISAT launch, the lower stratosphere content was probably at a historical low, making rather challenging the retrieval of aerosols. Nevertheless, Vanhellemont 


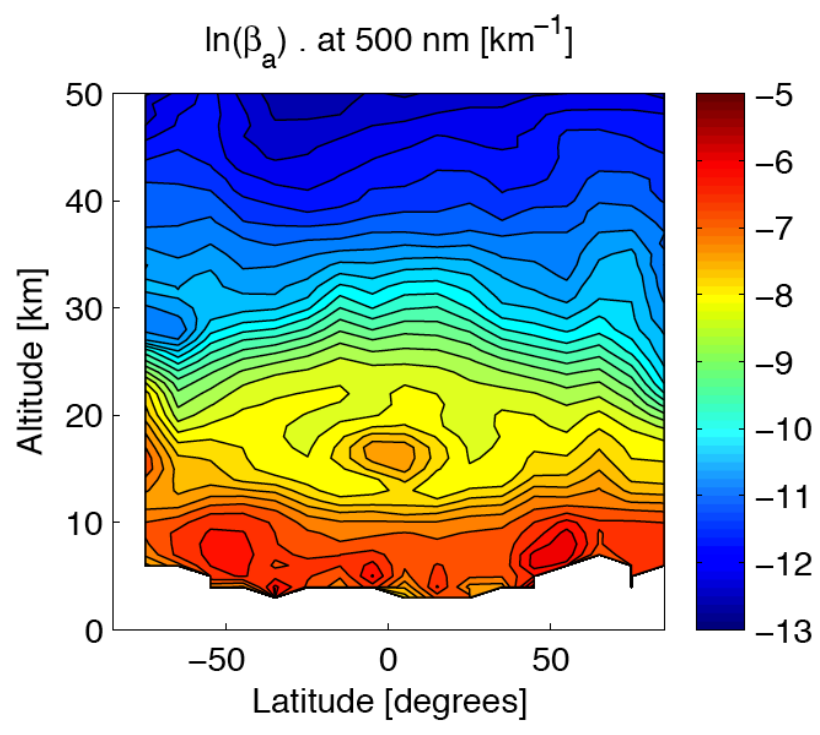

Fig. 43. The natural logarithm of the $500 \mathrm{~nm}$ aerosol extinction median values for the year $2003\left(\mathrm{~km}^{-1}\right)$. The isolated feature at the equator $(15-17 \mathrm{~km})$ is due to sub-visible equatorial cirrus, while PSCs are mostly seen in the South polar vortex, and barely visible in the North polar vortex (from Vanhellemont et al., 2005).

et al. (2005) established a first GOMOS climatology of aerosols and PSCs, based on the analysis of about 50000 night-time occultations during 2003, which allow probing at lower altitudes and with less noise due to bright-limb background contamination. In the fit of transmission spectra, it was decided to describe the aerosol extinction $\beta_{\mathrm{a}}(\lambda)$ with one parameter only:

$\beta_{\mathrm{a}}(\lambda)=N \frac{\sigma_{0}}{\lambda}$

with the fixed scaling factor $\sigma_{0}=3 \times 10^{-7} \mathrm{~cm}^{2} \mathrm{~nm}$ and $N$ (unit: $\mathrm{cm}^{-3}$ ) the parameter to be retrieved.

The climatology grid was composed of monthly bins, with 18 latitude bins with a width of 10 degrees, and an altitude grid with $1 \mathrm{~km}$ bins from 0 to $60 \mathrm{~km}$. No variations along longitude are considered (zonal mean). Finally, the median value in each bin was retained for establishing a climatology, and the variability was also studied. Figure 43 displays the median value for the whole year 2003 (all monthly bins averaged together) of the extinction $\beta_{\mathrm{a}}(\lambda)$ at $500 \mathrm{~nm}$.

Clearly visible is the umbrella-shaped aerosol layer, having the largest altitude above the equator and gradually sloping downwards when moving to the poles. The conspicuous isolated maximum in the tropics at an altitude of 15 to $17 \mathrm{~km}$ has been observed by other instruments as well and is caused by the presence of high sub-visible cirrus clouds in the tropics. As shown in Vanhellemont at al. (2005), this equatorial feature has a seasonal motion: along the year, it gradually moves towards the North, and then heads back southwards at the end of the year. This motion is in phase with the seasonal shift of the Intertropical Convergence Zone (ITCZ).

The PSCs revealed themselves in the elevated levels of the aerosol extinction in the Antarctic region, found confined to the polar vortex. On the contrary, no elevated extinction levels are observed in the Arctic region from January to March 2003 (and do not show in the yearly average). This may be explained by the much lower occurrence of PSCs, due to the weaker polar vortex. It should be noted that in this period there were some Sudden Stratospheric Warmings that weaken the polar vortex significantly.

A larger study, covering several years of GOMOS data (2002-2008), is presented in a companion paper of the ACP GOMOS Special Issue (Vanhellemont et al., 2010). In this case, based on a later version GOPR 6 of the GOMOS processing software, the wavelength dependence of the aerosol extinction is assumed to be of a quadratic form in $\lambda-$ $\lambda_{\text {ref }}, \lambda_{\text {ref }}=500 \mathrm{~nm}$ (Sect. 5.5.2). Four types of aerosols could be distinguished (from season, altitude and volcanic history, rather than from the spectral signature): PSCs, tropical subvisual cirrus, background aerosol, and volcanic stratospheric aerosols. Their typical vertical profiles are shown in Fig. 44, taken from Vanhellemont et al. (2010). The smoothness of these individual profiles is somewhat exaggerated, as a result of Tikhonov smoothing, yielding a $4 \mathrm{~km}$ target resolution. The effect of moderate volcanism is clearly seen in GOMOS data (Vanhellemont et al., 2010), and the dispersion of $\mathrm{H}_{2} \mathrm{SO}_{4}$ droplets made from volcanic $\mathrm{SO}_{2}$ reaching the stratosphere $(18-20 \mathrm{~km})$ could be followed, in particular the eruption of the Soufrière Hills volcano $\left(16.7^{\circ} \mathrm{N}, 62.2^{\circ} \mathrm{W}\right.$, Montserrat, West Indies), on 20 May 2006.

\subsection{Noctilucent clouds (Polar Mesospheric Clouds)}

Polar Mesospheric Clouds (PMCs) are thin clouds of ice particles condensing around $80-85 \mathrm{~km}$, which can be observed from the ground after sunset, while they are still illuminated by the sun: their first name was accordingly Noctilucent clouds. During a large number of star occultations, GOMOS has seen some extra signal coming from PMCs. In Fig. 45 is shown an example of GOMOS photometers' observation of a noctilucent cloud (or Polar Mesospheric Cloud), detected as a sharp increase of light. In addition to the star signal, and Rayleigh scattering by air molecules, there is some solar light scattered by the icy particles of the cloud.

A statistical analysis of PMC occurrence has been established (Pérot et al., 2010) over 4 years of data (bright limb only), from 2002 to 2006 . In order to condense, the icy particles need some water vapour and a very cold temperature, conditions found only in the high latitude summer mesosphere. The GOMOS data set consists of approximately 10000 cloud observations over the eight PMC 

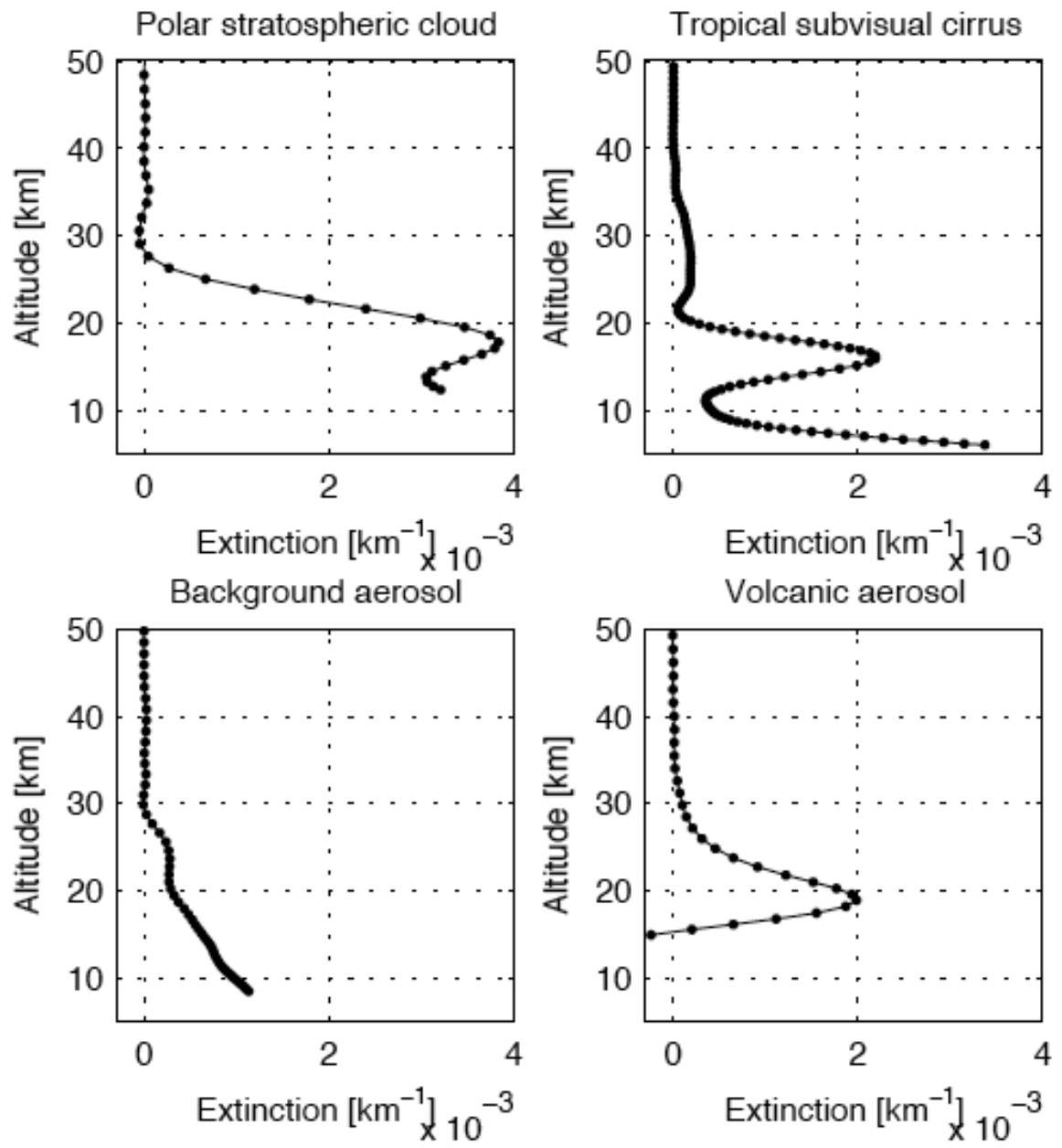

Fig. 44. Individual GOMOS particle extinction profiles: a Polar Stratospheric Cloud (30 July $2003,72.57^{\circ} \mathrm{S}, 2.18^{\circ} \mathrm{W}$ ), a tropical sub-visual cirrus cloud ( 7 October $2002,0.52^{\circ} \mathrm{S}, 78.69^{\circ} \mathrm{E}$ ), background stratospheric aerosols (12 September $2003,43.34^{\circ} \mathrm{S}, 137.36^{\circ} \mathrm{W}$ ) and volcanic stratospheric aerosols ( 9 January $2007,4.52^{\circ} \mathrm{S}, 27.15^{\circ} \mathrm{E}$ ). All plots have the same scale, in order to facilitate comparisons of magnitude (from Vanhellemont et al., 2010).

seasons studied. An example of cloud detection (or nondetection) is presented in Fig. 46 for the whole year 2004, for all day-side occultations. They are presented under the form of maps in Pérot et al., 2010. It is found that GOMOS photometers allow a very sensitive PMC detection. According to this first GOMOS climatology of PMCs, they seem to be more frequent in the Northern Hemisphere than in the Southern Hemisphere, confirming other observations. The number of stars for GOMOS available during the PMC season is about twice greater in the North than in the South in the latitude band $65^{\circ}-75^{\circ}$. The frequency of PMC occurrence, normalized by the number of observations, is $50 \%$ in the South and $80 \%$ in the North at the peak season (Pérot et al., 2010, Fig. 7).
The PMC altitude distribution was also calculated, and the obtained median values are $82.7 \mathrm{~km}$ in the North and $83.2 \mathrm{~km}$ in the South. It will be interesting to compare with the results of the Aeronomy of Ice in the Mesosphere (AIM) mission, recently launched (2007) by NASA, first satellite mission entirely dedicated to the study of polar mesospheric clouds (Russell III et al., 2009). It would allow extending the climatology of AIM with the years 2003-2006, before the launch of AIM, for a better study of long term trends. It has been suggested (Thomas and Olivero, 2001) that PMCs could be a potential indicator of global atmospheric changes: the increase of $\mathrm{CO}_{2}$ will decrease the temperature at these altitudes (by IR radiative cooling), while at the same time there might be an increase of water vapour injection in the stratosphere. There was indeed a magnificent PMC over Paris on Bastille Day (14 July) 2009, probably not sufficient to claim a climatic change indicator! 


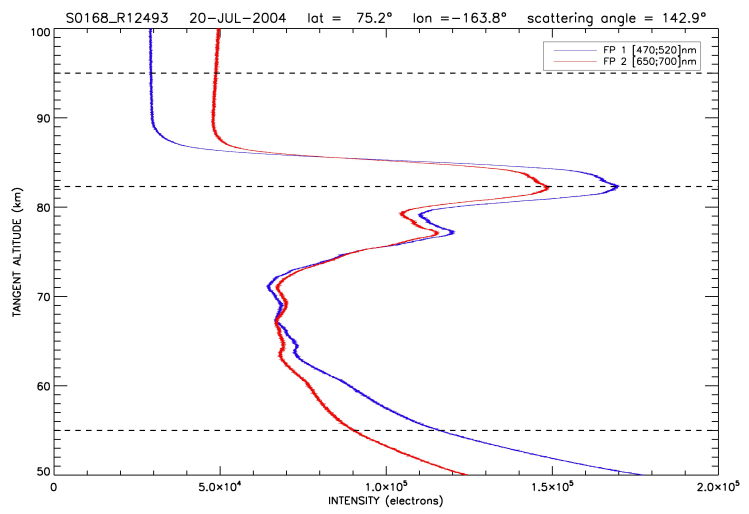

Fig. 45. Example of GOMOS photometers' observation of a noctilucent cloud (or Polar Mesospheric Cloud), detected as an increase of solar light scattered by the particles of the clouds, in addition to the Rayleigh scattering by air molecules around $85 \mathrm{~km}$. The dashed lines indicate, from top, the region with no cloud (signal from the star), the peak intensity of the cloud and the region below the cloud, where the Rayleigh scattering dominates the bright limb intensity (from Pérot et al., 2010).

The GOMOS spectrometers have also seen this PMC solar scattered light. The scattering efficiency as a function of wavelength will allow determination of the characteristics (at least the mean radius, and possibly the standard deviation of the size distribution width, assuming, for instance, a Gaussian distribution) of these icy particles.

\subsection{Detection and climatology of the natural sodium layer}

A layer of sodium atoms is present in the mesosphere, mainly as a result of ablation of meteorite material impacting the upper atmosphere. When illuminated by the sun, resonance scattering at the $589 \mathrm{~nm}$ doublet makes it the source of one of the most conspicuous natural aeronomic emissions, particularly for twilight observations (e.g., Bricard and Kastler 1944), and as such has been studied by a variety of methods (ground based spectrometers, lidars (Mégie and Blamont, 1977), space borne photometers on OGO-6 in 1969, etc.). It has recently attracted a renewed interest, because, besides the scientific questions related to the origin and the vertical distribution of the layer, the mesospheric sodium is of major importance in astronomy, where it is used to produce an artificial star on which to base guidance for adaptive optics with a lidar illumination.

Although extensive data exist about the mesospheric sodium layer measured from particular sites, very little is known about the geographical distribution (Plane, 2004). GOMOS has demonstrated a new method of studying the sodium layer, and has provided the first global mesospheric sodium climatology made so far (Fussen et al., 2004). The new method is to detect and measure the tiny absorption of the sodium doublet imprinted on the stellar spectrum during a standard star occultation. Many transmittance spectra have to be binned together in order to provide a sufficient accuracy. The bins are $1 \mathrm{~km}$ altitude, $10^{\circ}$ in latitude, and one month time. Figure 47 represent such a transmittance, the median of 1121 occultations in a particular bin. The two components of the Na doublet are well separated, but the line profile $(\sim 0.5 \mathrm{~nm})$ is the instrumental one, because the natural lines at mesospheric temperatures $(\sim 200 \mathrm{~K})$ are much narrower.

Fitting such a transmittance spectrum, one may retrieve an optical thickness averaged over the sampled wavelength interval and the slant (line) density of $\mathrm{Na}$ atoms as a function of altitude (Fig. 48, left). This vertical profile may be fitted by a layer of sodium atoms described by the peak altitude, the peak concentration, and two parameters describing the vertical width and some asymmetry w.r.t. the peak altitude.

The first analysis was based on about 100000 star occultations recorded during the year 2003 (Fussen et al., 2004). It was found later that, in some cases, the absorption lines were saturated, yielding an underestimate of the slant density of $\mathrm{Na}$ atoms. Once this effect was corrected for, Fussen et al. (2010) extended the GOMOS analysis to the whole period 2003-2009. The main findings are summarized below. For more details, see Fussen et al. (2010).

The peak concentration is variable, in the range $2--5 \times$ $10^{3}$ atoms $\mathrm{cm}^{-3}$. The integrated vertical column is in the range $1--7 \times 10^{9}$ atoms $\mathrm{cm}^{-2}$. The peak altitude varies in the range $90-93 \mathrm{~km}$, and the vertical extent is typically $\sim 15 \mathrm{~km}$. GOMOS data in the latitude bins $30-40^{\circ} \mathrm{N}$ and $40-50^{\circ} \mathrm{N}$ were compared to a series of lidar measurements acquired at Fort Collins (latitude $41^{\circ} \mathrm{N}$ ) (She et al., 2000). The results are generally consistent, but not fully identical.

GOMOS average climatology shows a strong enhancement of sodium at both polar winter solstices, with strong minima in between. At tropical latitudes, a semi-annual variation of the total column is present in the data, which evolves progressively at higher latitudes into an annual variation, a time-space distribution pattern unreported so far. The peak altitude demonstrates an annual variation with two peaks at mid-latitude, confirming earlier lidar results.

During polar winter the sodium layer peak altitude is lower, a sign that the layer is influenced by the mesospheric global circulation, ascending at the summer pole and descending at the winter pole around solstices.

It should be noted that, during summer polar solstices, the frequent presence of Polar Mesospheric Clouds (Perot et al., 2010) adds a strong signal at the limb, making more delicate the GOMOS retrieval of sodium. Future GOMOS studies will use also the bright limb emission of the $\mathrm{Na}$ doublet, in a fashion similar to the ODIN/OSIRIS bright limb observations (Gumbel et al., 2007). 


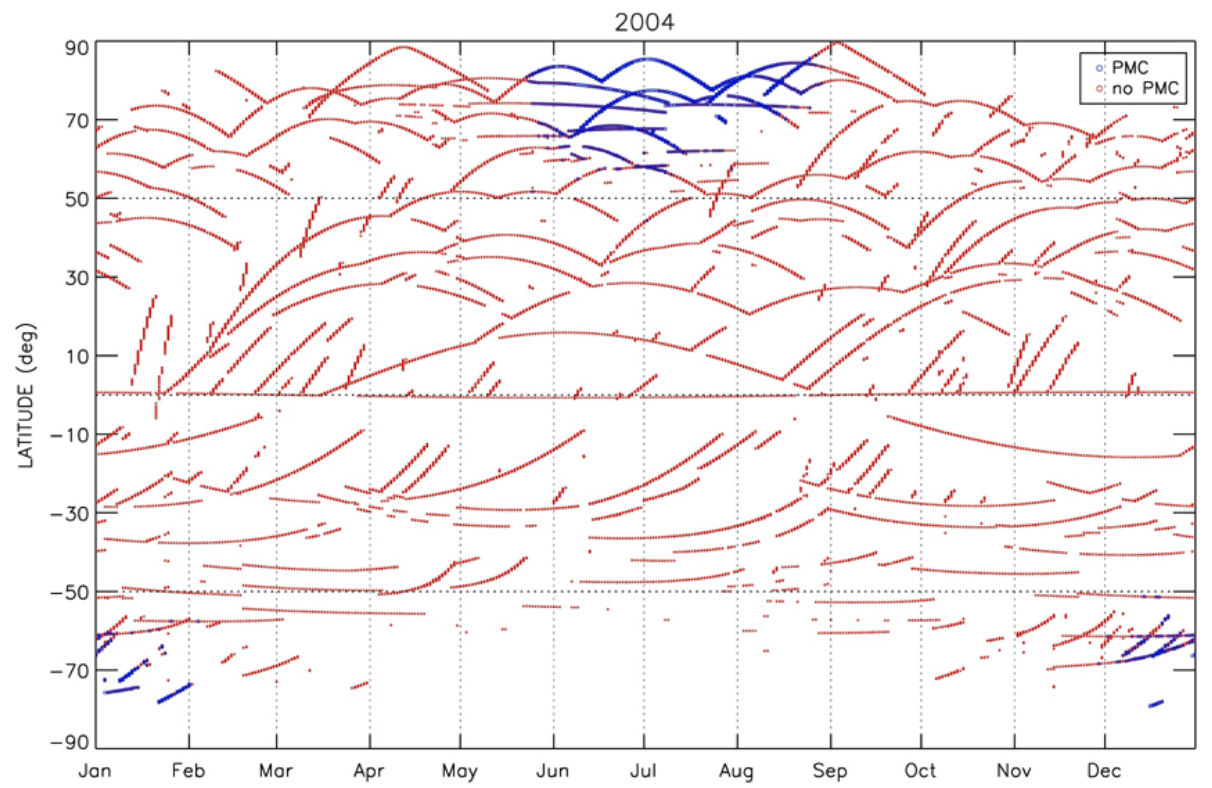

Fig. 46. Latitude-time plot of all day-side occultation points during the year 2004. In red are marked the points where no PMC is seen, while in blue are the points with a PMC detected. The seasonal behavior is well marked. The coverage is somewhat poorer in the south, but still PMCs are less frequent in the South than in the North.

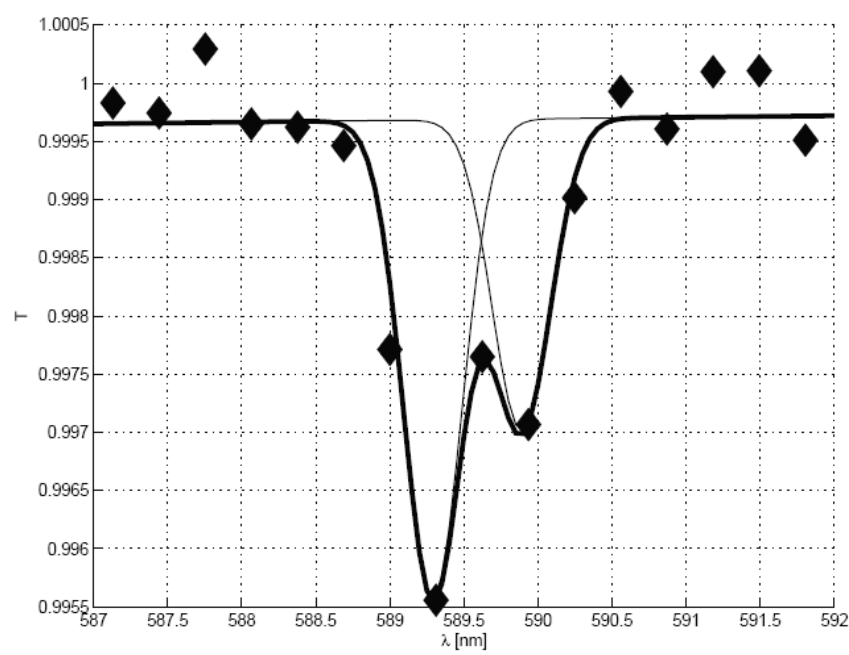

Fig. 47. Diamonds: the median transmittance (of 1121 occultations) measured between $70^{\circ} \mathrm{N}$ and $80^{\circ} \mathrm{N}$ during January 2003. Thin full lines: individual D2 and D1 absorptions, assumed to have the same instrumental line spread function. Thick line: best fit for the total transmittance (with baseline removed) (from Fussen et al., 2010).

\subsection{Scintillations and the study of air density irregularities: internal gravity waves and turbulence}

The scintillation of stars observed through the Earth's atmosphere is generated by random irregularities of air density (and, consequently, of refractive index). The two fast photometers of GOMOS (with red and blue filters), allows a statistical study of this field of irregularities with unprecedented refinement, and a global coverage for the first time.

As an example of the variability of observations, we show in Fig. 49 the signals of the red photometer as a function of tangent altitude, recorded during two different occultations. The signal is normalized to unity at high altitude. Both show increasing fluctuations with decreasing altitude, but the high latitude occultation $\left(62^{\circ} \mathrm{S}\right.$, black $)$ displays much stronger fluctuations than the other recorded at $37^{\circ} \mathrm{S}$ (red). The intensity of fluctuations may be quantified by one main parameter, the variance, $\beta^{2}$, of relative fluctuations of the measured signal; $\beta$ is defined as the scintillation index (Sofieva et al., 2007), and is displayed at bottom of Fig. 49. At high altitudes, the fluctuations are due to the noise, while at low altitudes, the fluctuations are saturated to unity in both occultations. The curves of $\beta(z)$ are otherwise very different between 30 and $70 \mathrm{~km}$, opening a prospect for case studies and climatology studies of density irregularities. The various analysis performed from GOMOS scintillation data are briefly reviewed below.

Gurvich and colleagues have proposed a model of the atmospheric density irregularities (i.e., Gurvich et al., 2005, and references therein) that is fully confirmed by the study of the signal of the two photometers $(1 \mathrm{kHz})$. In this theoretical forward model (going from the field of irregularities to a prediction of scintillations), the field of irregularities is the sum of two independent fields with different characteristics: 

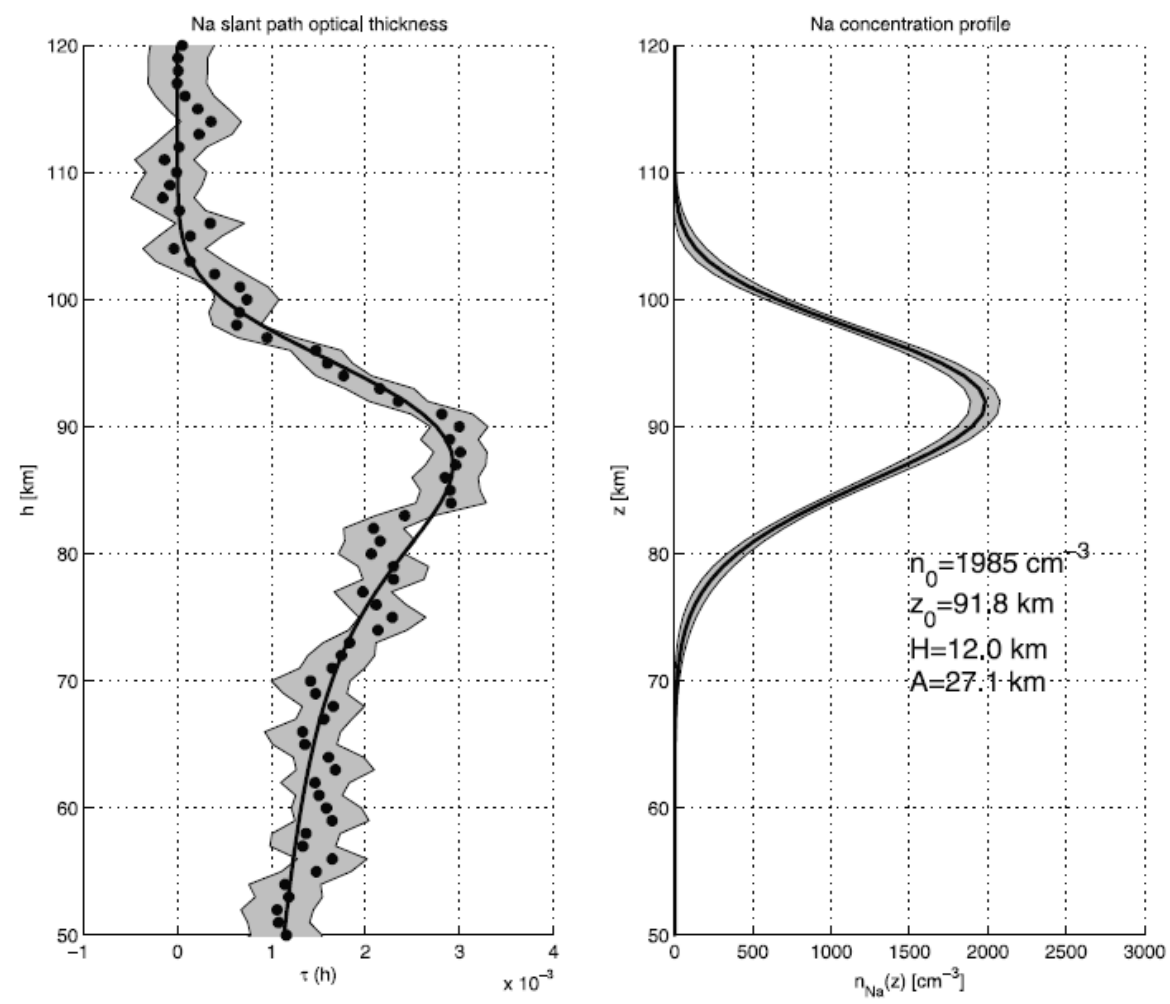

Fig. 48. Left panel: retrieved Na slant path optical thickness (full circles) versus the tangent altitude with associated errors (grey band). The full line is a best fit model. Right panel: corresponding Na concentration profile and optimal parameters. The grey band represents the estimated random error on the transmittance (Fussen et al., 2004).

- The anisotropic component represents mainly the Internal Gravity Waves (IGW). It is characterized by a spectrum (amplitude vs. size) with a large spectral slope $(-3)$ and a large aspect ratio (horizontal/vertical $\sim 100$ ).

- The isotropic component is characterized by its Kolmogorov ( $-5 / 3$ slope) isotropic spectrum. These density fluctuations are mainly representative of turbulence, which appear as a result of the breaking of gravity waves (but also from other mechanisms). It leads to effective turbulent mixing of chemicals in the stratosphere and to dissipation of kinetic energy into heat at the final step of this process, two key elements of stratospheric physics and chemistry.

Due to the exponential decrease of air density with altitude, the irregularities at the lowest altitude along the LOS produce the main contribution to the observed scintillations. Figure 50 is a sketch of such a field of irregularities with the two components, and the effect that it will have on the signal of the two fast photometers (FP). Blue rays are more refracted than red rays (since the index of refraction is larger), and therefore, from the observer at a given time, the blue ray is probing higher altitudes than the red ray. In the case of a perfectly vertical occultation (in the plane of the orbit), the two signals are probing the same irregularities (for both components), with a time delay between the two photometer signals increasing towards lower altitude. When the occultation is oblique with an angle $\alpha$ with the local vertical (Fig. 50, bottom), there is also a horizontal separation of the two trajectories of the LOS: the anisotropic component field will be similarly probed by the two photometers, because of the large horizontal extent of the irregularities, while the isotropic component will be completely different. Therefore, the correlation of the two signals will be much smaller for the isotropic component than for the anisotropic component. These photometers signals are displayed in Fig. 51 as a function of altitude in the case of a vertical occultation and for an oblique occultation, for a small slice of altitude of the tangent point $(250 \mathrm{~m})$. The correlation between the two signals is much larger for the vertical occultation. Gurvich et al. (2005) have calculated a "coherency" index which quantifies the correlation between the two signals.

The analysis of coherency was performed for 15 stellar occultations, in the range $20-50 \mathrm{~km}$. Below $20 \mathrm{~km}$, the signal is too low, and strong scintillations (multiple paths) may occur. Above $\sim 50 \mathrm{~km}$, the scintillations are generally too small. The analysis allowed the detection of layers 

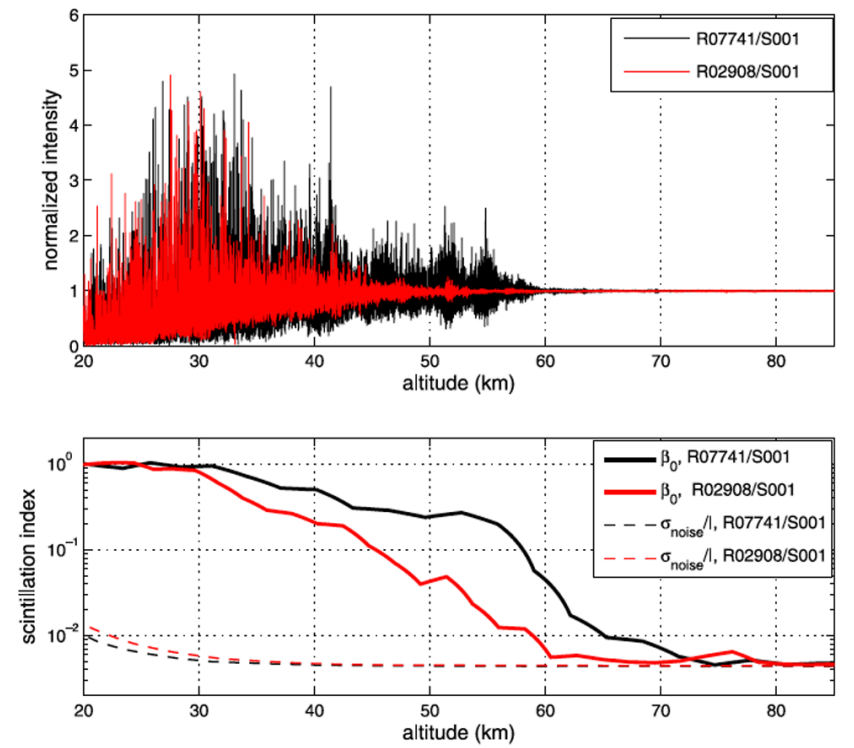

Fig. 49. Top: photometer signals by the GOMOS red photometers in occultations R07741/S001 $\left(62^{\circ} \mathrm{S}, 7^{\circ} \mathrm{E}, 23\right.$ August 2003, $23: 51 \mathrm{UTC})$ and R02908/S001 $\left(37^{\circ} \mathrm{S}, 106^{\circ} \mathrm{W}, 20\right.$ September 2002, 07:01 UTC). (bottom) Scintillation index $(\beta)$ for these two occultations (from Sofieva et al., 2007).

with prevailing isotropic component. They are located generally between the altitudes of about 30 and $40 \mathrm{~km}$. The thickness of the layers and their altitude distribution depend on observation location.

Overall, the model with the two components, IGW producing anisotropic structures (elongated horizontally) and smaller scale isotropic turbulence is validated by the observations in two colours. Once validated, Sofieva et al. (2007) established algorithms to determine the parameters of IGW and turbulence from the time-frequency spectral analysis of the GOMOS red photometer (preferred because of higher signal, more "focused" in refraction index, and less affected by absorption and scattering). Two retrieved parameters are $C_{W}$ and $C_{K}$, the structure characteristic defining the power of the anisotropic air density irregularities and the isotropic irregularities, respectively. The two other retrieved parameters are the inner and outer scales of the anisotropic component, found to increase with altitude from $\sim 5 \mathrm{~m}$ at $25 \mathrm{~km}$ to $15-25 \mathrm{~m}$ at $45 \mathrm{~km}$ (inner scale) and from $\sim 0.2-0.5 \mathrm{~km}$ at $25 \mathrm{~km}$ to $0.5-3 \mathrm{~km}$ at $45 \mathrm{~km}$ (outer scale). The value of $C_{K}$ is found to increase rapidly from 25 to $35 \mathrm{~km}$, and more slowly above. The structure characteristic of turbulent temperature fluctuations $C_{T}^{2}$ is related to $C_{K}: C_{T}^{2}=\langle T\rangle C_{K}$.

Based on about 8000 night-time occultations of the 30 brightest stars in 2003, Gurvich et al. (2007) have published the first global and seasonal distribution of the structure characteristic $C_{T}^{2}$ of isotropic turbulence at altitudes 30$50 \mathrm{~km}$. In each season and latitude bin, the median value
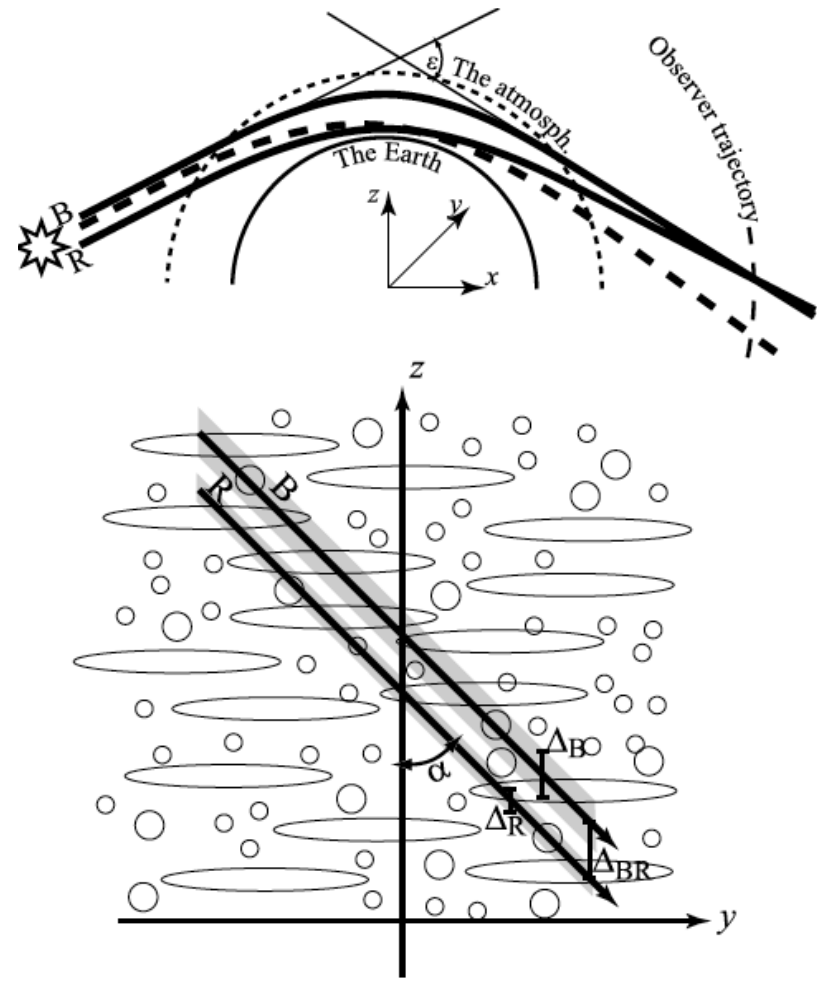

Fig. 50. Observation scheme of the two-wavelength scintillation. Top: a cut of the atmosphere by the plane containing B (blue) and $\mathrm{R}$ (red) rays (thick solid lines), which are received at the same time, $\mathrm{t} 0$, by the GOMOS fast photometers (FP). The ray curvature and the refraction angle $\varepsilon$ are largely exaggerated. Thick dashed line denotes a blue ray having the same ray perigee altitude as the red one, but coming to GOMOS FP with a refractive time delay dt. Bottom: a cut of the atmosphere by the atmospheric screen. Solid lines denote trajectories of the intersection points of the rays with this plane. Shading illustrates chromatic "thickness" $\Delta \mathrm{R}$ and $\Delta \mathrm{B}$ of the rays, due to the filters optical bandwidth. The photometers simultaneously register the rays with the same coordinate y. Sketched contours illustrate anisotropic and isotropic irregularities of air density (from Gurvich et al., 2005).

of $C_{T}^{2}$ (less sensitive to outliers) was kept. Large variations of $C_{T}^{2}$ are observed (Fig. 52): the range of derived values of $C_{T}^{2}$ covers more than two orders of magnitude, from $3 \times 10^{-5}$ to $6 \times 10^{-3} \mathrm{~K}^{2} \mathrm{~m}^{-2 / 3}$. The value of $C_{T}^{2}$ has a clear physical meaning. It is equal to the variance of the temperature difference at two points at a distance of one meter from one another. The largest $C_{T}^{2}$ values are observed above $40-45 \mathrm{~km}$ in polar winter in both hemispheres, but they are especially large in the Southern Hemisphere. Based on a simplified spectral analysis, Sofieva et al. (2007) suggested from a dedicated study of GOMOS scintillations that the strong turbulence observed at winter polar stratosphere might be related to breaking IGW in the polar night jet.

At low latitudes, a rather low turbulence is observed, with minimal values of $C_{T}^{2}$ less than $10^{-4} \mathrm{~K}^{2} \mathrm{~m}^{-2 / 3}$. Most interesting is the geographical distribution of $C_{T}^{2}$ displayed 

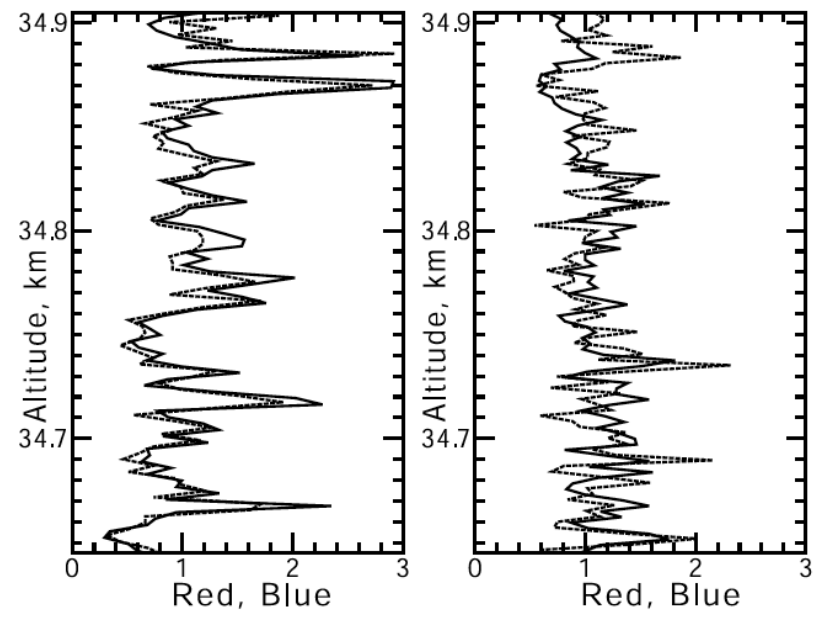

Fig. 51. Comparison of two records of two-wavelength scintillations ((left) vertical, (right) oblique at $\left.46^{\circ}\right)$. Abscissa is red $(672 \mathrm{~nm}$, dashed line) and blue $(499 \mathrm{~nm}$, solid line) intensities normalized on the corresponding mean. Ordinates are altitude of ray perigee. The correlation is poorer for oblique occultation, because of the effect of isotropic turbulence (from Gurvich et al., 2005).

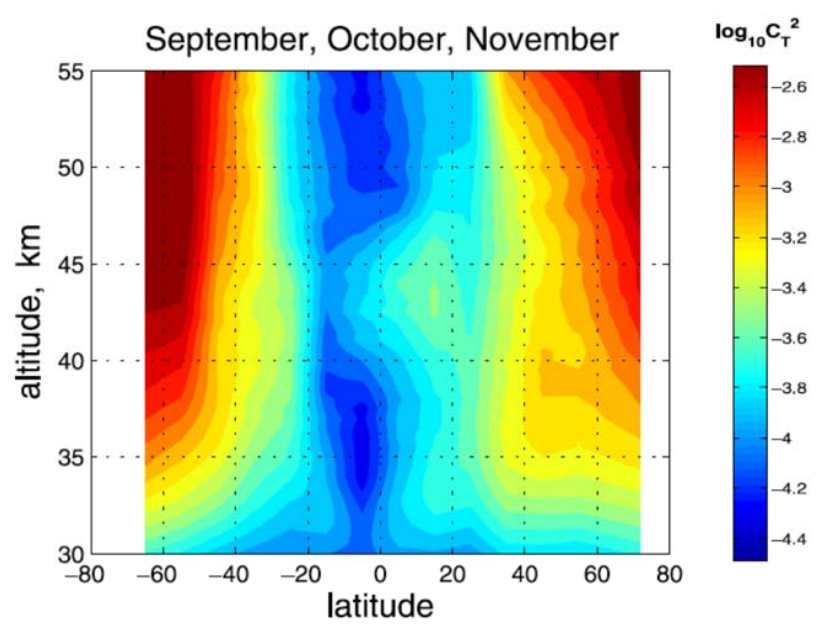

Fig. 52. Zonal averaged $C_{T}^{2}\left(\mathrm{~K}^{2} \mathrm{~m}^{-2 / 3}\right)$ in one season, which covers the end of southern winter pole (September) and the beginning of Northern winter pole (November) logarithmic color scale. It shows the large increase of $C_{T}^{2}$ in both winter poles, connected to the polar night jet. The four seasons are displayed in Gurvich et al. (2007).

on Fig. 53, in a layer about $3 \mathrm{~km}$ thick, centered around $\sim 42 \mathrm{~km}$, between $35^{\circ} \mathrm{N}$ and $35^{\circ} \mathrm{S}$. Turbulence intensity is smaller in this zone than in polar regions and, in average, it has a pronounced zonal structure. The plots of Fig. 53 show that the average values of $C_{T}^{2}$ follow the sub-solar latitude, as do the main features of the general circulation of the middle atmosphere (Holton, 2004). Also, almost all of the local enhancements are over continents (except for the one over Western Pacific in June-September). Many of $C_{T}^{2}$ enhancements seen in Fig. 53 correspond well to the typical regions of deep convection, e.g., Africa, South America and Indonesia in December, January-March, regions of Indian and American monsoons and of North African dry convection in June-September.

No systematic increase of $C_{T}^{2}$ over large mountain ranges such as the Himalaya, Andes and Sierra Nevada are seen: turbulence enhancements in tropical regions are not related to orography. Probably mountain wave wavelengths are too long for transformation into the small scale turbulence observed by scintillation at $42 \mathrm{~km}$ (Gurvich et al., 2007). Various hypothesis for the origin of the observed pattern were discussed (Gurvich et al., 2007) such as the general circulation, forced by solar heating, or a remote consequence of the deep convection. A similar structure was also reported (Jiang et al., 2004) for the temperature variance around $38 \mathrm{~km}$ as observed by MLS, suggesting a relation with gravity wave breaking.

More recently, Sofieva et al. (2009) have refined the forward model and retrieved additional information about gravity waves: their potential energy per unit mass $E_{\mathrm{p}}$ (Joule $\mathrm{kg}^{-1}$ ), which depends on the outer scale of the GW. The new algorithms are implemented at FMI and in the GPOD (Grid Processing-On-Demand) environment at the European Space Agency). In Fig. 54 are shown the latitude/altitude distribution of $E_{\mathrm{p}}$ zonal mean for two 4-month periods in 2003: January-March plus December (2002), and June-September. The potential energy, $E_{\mathrm{p}}$, increases with altitude. Enhancements in equatorial regions are clearly observed, analogous to those found in global analysis of radio-occultation data and caused by large values of GW outer scale at these locations. Enhanced $E_{\mathrm{p}}$ in the $\mathrm{SH}$ winter high latitude stratosphere is observed, but no symmetric enhancement of $E_{\mathrm{p}}$ at high latitudes in winter is observed in Northern Hemisphere. Sofieva et al. (2009) suggested that it is probably caused by the fact that occultations at $\mathrm{NH}$ winter high latitudes were carried out in January 2003 and in December 2003, when some sudden stratospheric warmings (SSW) lasted a significant part of the observational period. It coincides with previous lidar observations (Whiteway and Carswell, 1994), showing reduced GW activity during SSW.

Future multi-year analysis of GOMOS scintillation measurements will refine the observations and will provide information about spatial and inter-annual variability of GW and turbulence spectra parameters in the stratosphere.

\subsection{High-resolution density and temperature profiling using bi-chromatic scintillation}

As explained above, density irregularities produce scintillations that are observed with the two photometers in two colors. The two photometers were implemented in the design of GOMOS for a better correction of the stellar 


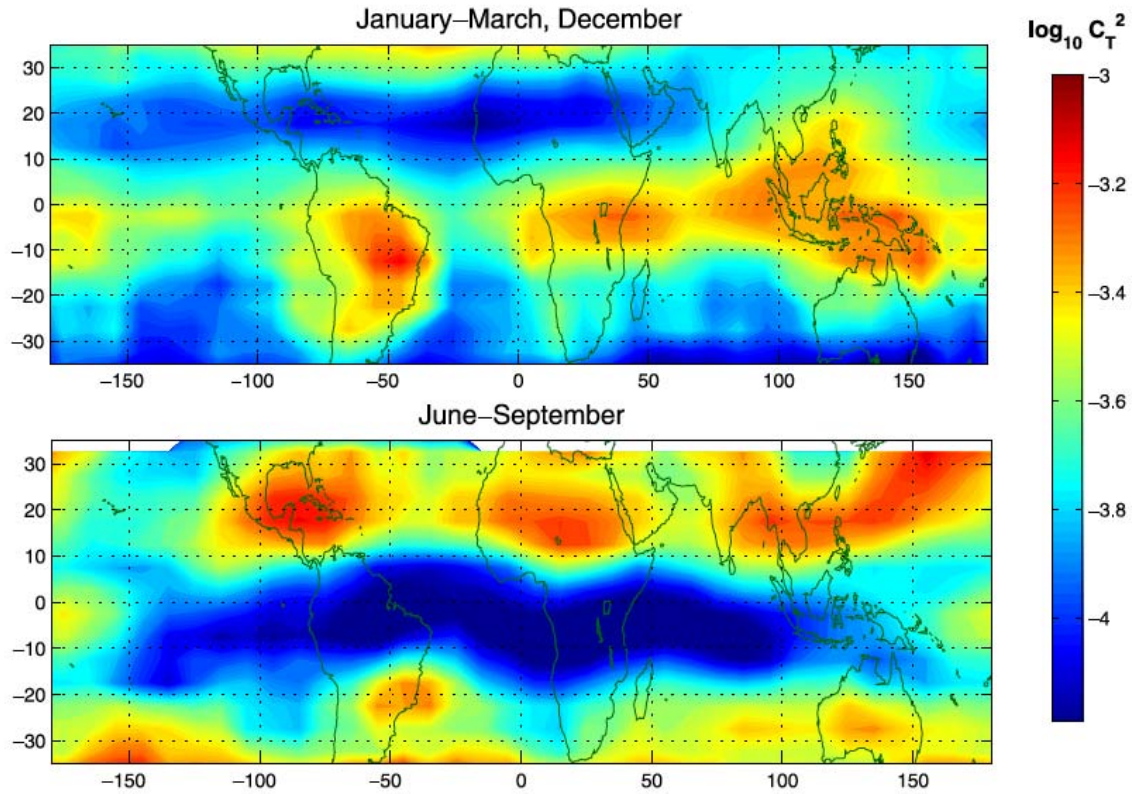

Fig. 53. $C_{T}^{2}\left(\mathrm{~K}^{2} \mathrm{~m}^{-2 / 3}\right)$ at $42 \mathrm{~km}$ for January-March (top), Dec and for June-September (bottom), logarithmic color scale. The data are averaged in $5^{\circ} \times 10^{\circ}$ latitude-longitude bins and a three-point smoothing is applied to the obtained fields. In order to make these maps more contrasting, the color scale covers one decade only (from Gurvich et al., 2007).
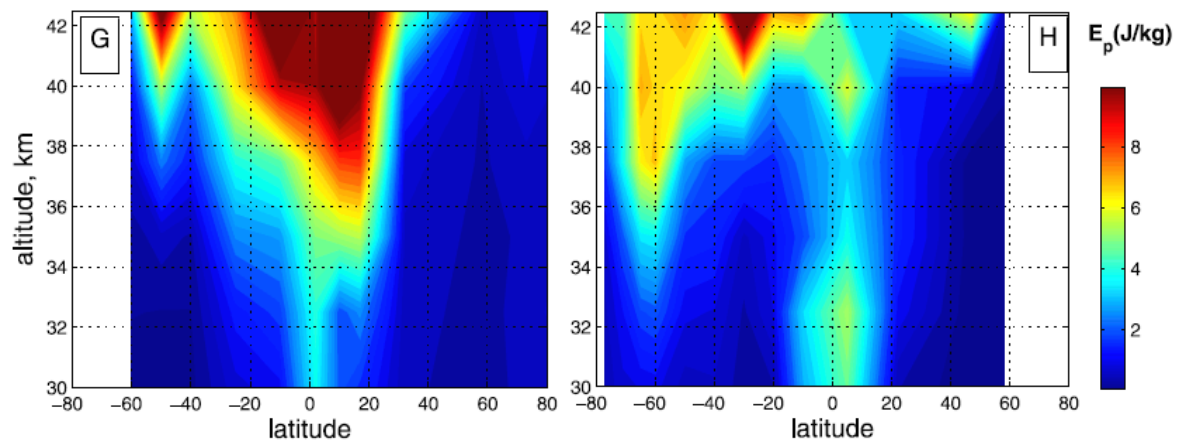

Fig. 54. Zonal mean values of the potential energy $E_{\mathrm{p}}$ (Joule $\mathrm{kg}^{-1}$ ) of gravity waves as determined from GOMOS scintillation measurements during two 4 months periods of year 2003: January-March plus December (left), and June-September (right). See text for discussion (from Sofieva et al., 2009).

spectra distorted by refraction. However, it turned out that another use of the two photometers was possible, not anticipated before launch: the retrieval of density and temperature profiles with a high vertical resolution, from the measured time delay between red and blue scintillation peaks corresponding to the same density irregularity. Dalaudier et al. (2006) have developed the so-called HRTP (High Resolution Temperature Profile) method and algorithms to determine density and temperature profiles between 20 and $30 \mathrm{~km}$ with a vertical resolution of $200 \mathrm{~m}$. In this altitude range the lower stratosphere is not sufficiently sampled by in situ measurements while meteorological satellites cannot provide vertical resolution better than $\sim 2 \mathrm{~km}$. Figure 55 shows a sample of the two photometers signals over $200 \mathrm{~m}$. The bending refraction angle may be determined from the time delay, which is related to refractivity, therefore density at the tangent point. Then the inversion method is similar to a classical radio occultation involving the inversion of an Abel's integral, the hydrostatic equilibrium and the ideal gas law.

Figure 56 shows the result of such an inversion, selected because it was not far from a lidar measurement at Mauna Loa, for comparison purposes. Both lidar and GOMOS show similar, and significant departures from the ECMWF profile that is inherently smooth (up to $6 \mathrm{~K}$ ), while the difference GOMOS HRTP - Lidar does not exceed $\pm 2 \mathrm{~K}$ above $22 \mathrm{~km}$. 


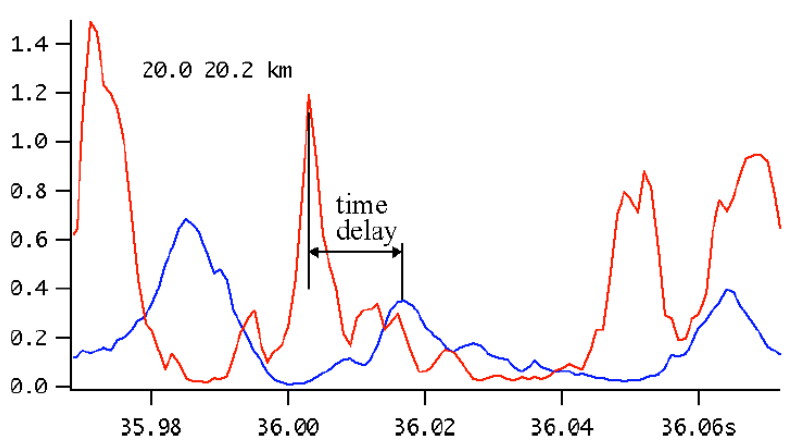

Fig. 55. Sample of blue and red photometer records (103 milliseconds only). The tangent altitude is in the range 20.2$20.0 \mathrm{~km}$ ( $200 \mathrm{~m}$ vertical range). Note the time delay and the additional smoothing of the blue signal due to larger refractivity bandwidth. Absorption of blue light is stronger due to Rayleigh and aerosol scattering (Dalaudier et al., 2006).

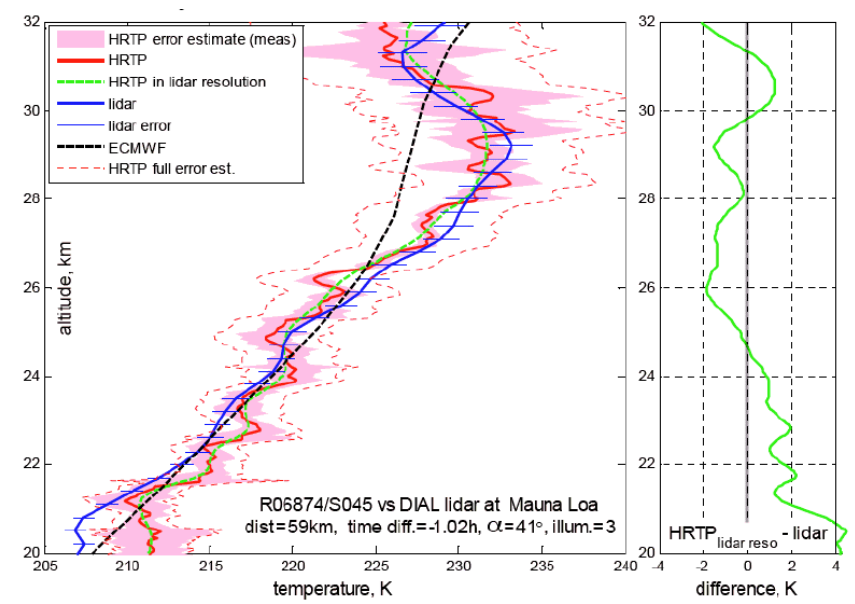

Fig. 56. A sample HRTP temperature profile (in red) obtained from two-color scintillation data. The uncertainty (in pink) distinguishes contribution from random errors (colored area) and total error including initialization bias (dashed). From Dalaudier et al. (2006).

We do expect such differences, because the two measurements do not coincide exactly in time and space, while the characteristics of gravity waves are such that ideally the two measurements should be separated by less than $20 \mathrm{~km}$ and less than 2 to $3 \mathrm{~h}$.

While validation with precisely collocated measurements is difficult, as shown by Sofieva et al. (2008) (i.e., the balloon-borne radiosonde takes $\sim 1 \mathrm{~h}$ to explore up to $30 \mathrm{~km}$ ), it is possible to validate HRTP results by comparing the power spectra of temperature fluctuations vs size, as done by Sofieva et al. (2009d). These authors have compared a subset of GOMOS spectra to radiosonde spectra, where the requirements on space and time collocation are much less stringent. Overall, the HRTP results were validated and it was found that the altitude resolution was $150 \sim 200 \mathrm{~m}$.
This new HRTP method will be implemented in the GOMOS data processing chain in the next release (produced with IPF 6), providing a new product of great interest. It should be emphasized that not only the fluctuations of temperature are recorded with unprecedented vertical resolution from space, but also the absolute value of the temperature is retrieved with a good accuracy, making this product a good candidate for future assimilation in meteorological analysis like ECMWF.

\section{Conclusions}

We have presented the rationale of GOMOS design, the instrument description, some "raw" observations, and an overview of results obtained up to now. A large number of scientific research activities may still be done with the GOMOS data, in the UTLS, the stratosphere and the mesosphere.

As said earlier, the example of the study of $\mathrm{NO}_{3}$ coupled with temperature, $\mathrm{NO}_{2}$ and $\mathrm{O}_{3}$ (Sect. 7.7) illustrates how the stratosphere itself may be used as a gigantic laboratory to determine chemical reaction rates.

In a similar way, the rate constants of many other reactions of stratospheric pertinence could be adjusted, by comparing GOMOS measurements (or others) to a prediction of a chemistry transport model where the dynamics are forced by a meteorological field like ECMWF, and where the GOMOS measurements would be assimilated. A systematic bias between Observed and Forecast would be the sign that something has to be changed in the description of the model, which relies on somewhat uncertain reaction rates. Unknown dynamical effects could also be detected.

The 4 years nominal lifetime of ENVISAT has now been exceeded at 7 years. Still, it may be a little bit short for trend studies. The collection of several years of GOMOS measurements, because of their absolute character, will provide a unique benchmark of data, to be compared to previous measurements sharing also this absolute character, and most over, to future measurements collected by the same method of stellar occultations, for long term monitoring. In this respect we hope that GOMOS is the first of a series of stellar occultations monitoring instruments.

The actual size and complexity of GOMOS is in large part due to a number of precautions and redundancies that were included in its design, because of its exploratory character of a new technique. That which has been learned from this first large-scale investigation of the stellar occultation technique will allow a significant simplification of the design of future instruments, with a smaller mass and complexity. For instance, the use of a dedicated micro-satellite would be quite adapted to such an investigation. The whole spacecraft might be oriented to each successive star, as is done for SPICAM and SPICAV instruments, respectively around Mars and Venus at present (Bertaux et al., 2006, 2007). It would be 
recommended to cool the CCD detector to at least $-40^{\circ} \mathrm{C}$, in order to make the dark charge negligible, even after radiation damage. Also, a polar orbit is recommended for latitude coverage, but the altitude could be lower, avoiding the problem of radiation contamination in the South Atlantic Anomaly.

\section{Appendix A}

\section{A short history of the stellar occultation technique}

The use from space of the stellar occultation technique has been reviewed by Smith and Hunten (1989). It was first discussed in details by Hays and Roble (1968) in a seminal paper, and used to measure terrestrial ozone from the astronomical satellites OAO-2 and Copernicus (OAO-3). Using OAO-2 in 1970 and 1971, Hays and Roble (1973) detected directly for the first time the mesospheric ozone maximum (from UV absorption of the stellar light) which had been previously inferred by Evans and Llewellyn (1970) from rockets sounding of the $\mathrm{O}_{2}\left({ }^{1} \Delta\right)(1.27 \mu \mathrm{m})$ and $\mathrm{O}_{2}\left({ }^{1} \Delta\right)(760 \mathrm{~nm})$ airglow emission, produced from photodissociation of ozone. Later, in 1975 and 1976 the Orbiting Astronomical Observatory $n^{\circ} 3$ (OAO-3, soon renamed Copernicus) was also used to measure successfully terrestrial ozone above $45 \mathrm{~km}$ form UV absorption in a wavelength region similar to OAO-2 (Riegler et al., 1976, 1977). The problem is that Copernicus retrieved ozone densities in the stratosphere (say, at $50 \mathrm{~km}$ ) that were found to be in serious discrepancy with model predictions and, more important, with OAO-2 results. Copernicus $\mathrm{O}_{3}$ densities were 2 to 3 times larger than model predictions, and about 25 times larger than the OAO-2 results. This enormous discrepancy, which is unresolved up to now (Atreya et al., 1981), did not help to promote the validity of stellar occultation technique as a reliable method for ozone measurements, and this is perhaps the reason for which it was not pursued further in the USA, in spite of its inherent merits.

After careful re-reading of the scientific papers, and with the experience accumulated with GOMOS analysis, we may here suggest a new explanation of this discrepancy. Clearly, both OAO-2 and Copernicus results could be reconciled with each other, and with models, by a shift in altitude of the ozone retrieved profiles: about $10 \mathrm{~km}$ upwards for OAO-2, and $5 \mathrm{~km}$ downwards for Copernicus. Such errors are in principle incompatible with orbital knowledge accuracy, but there is another source of error in the retrieved measurement altitude: the direction of the star in the sky. Indeed, astronomical directions of stars are described in a conventional system with the origin of RA (right ascension) as it was in a given year (so-called J1950 or now, J2000), which rotates with the precession of equinoxes by $360^{\circ}$ in 26000 years, while orbital computations of Earth satellites are most often using the inertial Earth equatorial system
True of date (with the origin of RA at its position at the time considered). This is because satellite tracking stations are fixed to the Earth, and need to know the coordinates of the spacecraft in a system linked to the Earth. Of course, it is trivial to include the correction of equinox precession in the calculation of the retrieved altitude of occultation measurements, but it has been sometimes overlooked in the past (for example, during the first light of Hubble Space Telescope), or even applied wrongly twice to the calculations (e.g. the SOLSTICE instrument on UARS and SORCE, G. Rottman, personal communication, 2003). An error in the position of the star will translate in an error of retrieved altitude which depends on the star position, the orbit altitude, and the particular geometry of occultation. For measurements made in 1975, the precession is at maximum w.r.t. $\mathrm{J} 1950$ or $\mathrm{J} 2000$, and amounts to $0.36^{\circ}$, translating in an altitude error (both ways) of up to $16 \mathrm{~km}$ depending on the particular orientation of the occultation geometry.

All these early attempts of stellar occultations suffered from the fact that only one wavelength was monitored during the occultation, giving room for confusion on the exact nature of the absorbing species. One great progress for the occultation technique w.r.t. the description of Hays and Roble was the use of multi-pixel detectors. The advent of CCDs (or other multi-pixels detectors), enabling measurement of the whole transmission spectrum at once, increased dramatically the capability of stellar occultation versus the use of filters, or scanning spectrometers. The first multi-pixel occultation (to our knowledge) was inaugurated with the ultraviolet spectrometer UVS placed on board the Voyager 1 and 2 missions: in 1979, an occultation of the star alfa Leonis by the atmosphere of Jupiter was observed from a distance of 14 millions $\mathrm{km}$ by Voyager 1 in the full range $110-170 \mathrm{~nm}$ allowing one to probe the $\mathrm{H}_{2}$ and methane vertical profiles (Festou et al., 1981). Since then, this technique was fostered at Service d'Aéronomie, in particular for the study of the atmosphere of the planet Mars with the SPICAM instrument. A first instrument dedicated to multi-wavelength stellar occultations was destroyed with the launch failure of the Russian mission Mars 96 (in November 1996), while a smaller version, SPICAM Light, is now flying on board Mars Express ESA mission (Bertaux et al., 2006), and around Venus (SPICAV) with Venus Express ESA mission (Bertaux et al., 2007).

Before GOMOS and its $\sim 600000$ occultations up to 2009, a big step forward was made with MSX mission, with about 200 occultations recorded around the Earth (Yee et al., 2002). The Cassini mission should also be mentioned, with very successful star occultations by the atmosphere of Titan performed at each encounter with UVIS spectrometer. With its extended atmosphere, Titan is the ideal planet for the technique of stellar occultation, where 6 organic molecules were identified from their UV absorption spectral signature (Shemansky et al., 2005). 
In the past, a number of stellar occultations have been also performed successfully from a balloon platform, allowing in particular the first observation of $\mathrm{NO}_{3}$ from a balloon, and the retrieval of its vertical distribution (Naudet et al., 1989; and more recently for $\mathrm{NO}_{2}$, Renard et al., 1997b). It should be mentioned that stars may be used also from the ground, as in the instrument developed for Antarctic measurements by Roscoe et al. (1997).

Acknowledgements. ENVISAT is a space mission of Earth Observation Programme of ESA, and GOMOS has been built by EADS Astrium under the direction and funding from ESA. We acknowledge the important contributions of Guy Brasseur and other scientists to the original proposal to ESA. We are particularly grateful to Jacques Louet and Guido Levrini from ESTEC for their efforts to establish the ENVISAT programme, to Alex Popescu, Thorgeir Paulsen at ESTEC, Alain Clochet, Jacques Dauphin and Patrick Uguen at Astrium, for managing the construction of GOMOS, Nicole Papineau and Carole Deniel at CNES, and national funding agencies from France (CNES, CNRS-INSU), Finland (TEKES), and Belgium for supporting partially the data analysis. We are grateful to many GOMOS SAG members, in particular Howard Roscoe, for a number of intense discussions. We thank also the control team at ESOC for conducting the GOMOS operations, and Henri Laur and Pascal Lecomte at ESRIN. We wish to thank also Nadège Montoux for providing some additional informations on water vapor comparison.

Edited by: P. Bernath

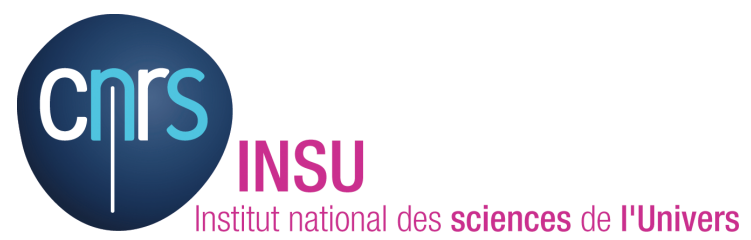

The publication of this article is financed by CNRS-INSU.

\section{References}

Amekudzi, L. K., Bramstedt, K., Rozanov, A., Bovensmann, H., and Burrows, J. P.: Retrieval of Trace Gas Concentrations from Lunar Occultation Measurements with SCIAMACHY on ENVISAT, New Horizons in Occultation Research, Studies in Atmosphere and Climate, Andrea Steiner, Barbara Pirscher, Ulrich Foelsche and Gottfried Kirchengast, 87-96, doi:10.1007/978-3-642-00320, 2009.

Atreya, S. K.: Measurement of minor species $/ \mathrm{H}_{2}, \mathrm{Cl}, \mathrm{O}_{3}, \mathrm{NO} / \mathrm{in}$ the earth's atmosphere by the occultation technique, Adv. Space Res., 1, 127A, 1981.

Atreya, S. K., Wasser, B., Donahue, T. M., Sharp, W. E., Drake, J. F., and Riegler, G. R.: Ultraviolet stellar occultation measurement of the $\mathrm{H}_{2}$ and $\mathrm{O}_{2}$ densities near $100 \mathrm{~km}$ in the earth's atmosphere, Geophys. Res. Lett., 3, 607-610, 1976.

Austin, J., Shindell, D., Beagley, S. R., Brhl, C., Dameris, M., Manzini, E., Nagashima, T., Newman, P., Pawson, S., Pitari, G., Rozanov, E., Schnadt, C., and Shepherd, T. G.: Uncertainties and assessments of chemistry-climate models of the stratosphere, Atmos. Chem. Phys., 3, 1-27, doi:10.5194/acp-3-1-2003, 2003.

Bates, D. R. and Nicolet, M.: Atmospheric hydrogen, Publ. Astron Soc Pacific, 62, 106-110, 1950.

Bertaux, J. L.: GOMOS Mission objectives, in: ESAMS99, European Symposium on Atmospheric Measurements From Space, ESA, Noordwijk, WPP-161, 79-87, 1999.

Bertaux, J. L., Pellinen, R., Simon, P., Chassefière, E., Dalaudier, F., Godin, S., Goutail, F., Hauchecorne, A., Le Texier, H., Mégie, G., Pommereau, J. P., Brasseur, G., Kyrölä, E., Tuomi, T., Korpela, S., Leppelmeier, G., Visconti, G., Fabian, P., Isaksen, S. A., Larsen, S. H., Stordahl, F., Cariolle, D., Lenoble, J., Naudet, J. P., and Scott, N.: GOMOS, proposal in response to ESA EPOP-1, A.O.1, January, 1988.

Bertaux, J. L., Mégie, G., Widemann, T., Chassefiere, E., Pellinen, R., Kyrölä, E., Korpela, E., and Simon, P. C.: Monitoring of ozone trend by stellar occultations: the Gomos instrument, Adv. Space Res., 11(3), 237-242, 1991.

Bertaux, J. L., Kyrölä, E., and Wehr, T.: Stellar Occultation Technique for Atmospheric Ozone Monitoring: GOMOS on Envisat, Earth Observation Quarterly, 67, 17-20, 2000.

Bertaux, J. L., Dalaudier, F., Hauchecorne, A., Chipperfield, M., Fussen, D., Kyrölä, E., Leppelmeier, E., and Roscoe, H.: Envisat: GOMOS-An instrument for global atmosphere ozone monitoring, edited by: Harris, R. A., ESA SP-1244, 109 pp., May 2001.

Bertaux, J. L., Hauchecorne, A., Dalaudier, F., Cot, C., Kyrölä, E., Fussen, D., Tamminen, J., Leppelmeier, G. W., Sofieva, V., Hassinen, S., d'Andon, O. F., Barrot, G., Mangin, A., Théodore, B., Guirlet, M., Korablev, O., Snoeij, P., Koopman, R., and Fraisse, R.: First results on GOMOS/Envisat, Adv. Space Res., 33, 1029-1035, 2004.

Bertaux, J. L., Korablev, O., Perrier, S., Quémerais, E., Montmessin, F., Leblanc, F., Lebonnois, S., Rannou, P., Lefèvre, F., Forget, F., Fedorova, A., Dimarellis, E., Reberac, A., Fonteyn, D., Chaufray, J. Y., and Guibert, S.: SPICAM on Mars Express: Observing modes and overview of UV spectrometer data and scientific results, J. Geophys. Res., 111, E10S90, doi:10.1029/2006JE002690, 2006.

Bertaux, J. L., Vandaele, A.-C., Korablev, O., Villard, E., Fedorova, A., Fussen, D., Quémerais, E. , Belyaev, D., Mahieux, A., Montmessin, F., Muller, C., Neefs, E., Nevejans, D., Wilquet, V., Dubois, J. P., Hauchecorne, A., Stepanov, A., Vinogradov, I., Rodin, A., and the SPICAV/SOIR team: A warm layer in Venus' cryosphere and high-altitude measurements of $\mathrm{HF}, \mathrm{HCl}, \mathrm{H}_{2} \mathrm{O}$ and HDO, Nature, 450, 646-649, doi:10.1038/nature05974, 2007.

Bertaux, J. L., Hauchecorne, A., Montoux, N., Dalaudier, F., Blanot, L., Lebrun, J. C., Kyrölä, E., Sofieva, V., Fussen, D., Barrot, G., Fehr, T., and Saavedra de Miguel, L.: Water vapor measurements in the stratosphere at $936 \mathrm{~nm}$ by stellar occultations with GOMOS/Envisat, Proceedings Atmospheric Science Conference, Barcelona, Spain, 7-11 Sep 2009, ESA Special Publication SP-676, 2009.

Borchi, F. and Pommereau, J.-P.: Evaluation of ozonesondes, HALOE, SAGE II and III, Odin- OSIRIS and -SMR, and ENVISAT-GOMOS, -SCIAMACHY and -MIPAS ozone profiles in the tropics from SAOZ long duration balloon measurements in 2003 and 2004, Atmos. Chem. Phys., 7, 2671-2690, doi:10.5194/acp-7-2671-2007, 2007. 
Bovensmann, H., Burrows, J .P., Buchwitz, M., Frerick, J., Noël, S., Rozanov, V. V., Chance, K. V., Goede, A. P. H., SCIAMACHY: Mission Objectives and Measurements modes, J. Atmos.Sciences, 56(2), 127-149, 1999.

Bracher, A., Bovensmann, H., Bramstedt, K., Burrows, J.P., Clarmann, T. V., Eichmann, K.-U., Fischer, H., Funke, B., GilLopez, S., Glatthor, N., Grabowski, U., Höpfner, M., Kaufmann, M., Kellmann, S., Kiefer, M., Koukouli, M. E., Linden, A., Lopez-Puertas, M., Tsidu, G. M., Milz, M., Noel, Rohen,G., Rozanov, A., Rozanov, V. V., Savigny, C. V., Sinnhuber, M., Skupin, J., Steck, T., Stiller, G. P. S., Wang, D.-Y., Weber, M., and Wuttke, M. W., Cross comparisons of $\mathrm{O}_{3}$ and $\mathrm{NO}_{2}$ measured by the atmospheric ENVISAT instruments GOMOS, MIPAS, and SCIAMACHY, Adv. Space Res., 36, 855-867, 2005.

Brasseur, G. and Solomon, S.: Aeronomy of the Middle Atmosphere, Chemistry and Physics of the stratosphere and mesosphere, 3rd revised and enlarged edn., Springer, Germany, 644 pp., 2005.

Crutzen, P. J.: Ozone production rates in oxygen-hydrogen-nitrogen oxide atmosphere, J. Geophys Res., 76, 7311-7327, 1971.

Dalaudier, F., Kan, V., and Gurvitch, A. S., Chromatic refraction with Global Ozone Monitoring by occultation of stars, I. Description and scintillation correction, Appl. Optics, 40(6), 866-877, 2001.

Dalaudier, F., Sofieva, V., Hauchecorne, A., Kyrölä, E., Laurent, L., Marielle, G., Retscher, C., and Zehner, C.: High-Resolution Density and Temperature Profiling in the Stratosphere Using BiChromatic Scintillation Measurements by GOMOS, Proceedings of the First Atmospheric Science Conference, European Space Agency, 2006, edited by: Lacoste, H. and Ouwehand, L., ESA SP-628, Published on CDROM, p. 34.1., ISBN92-9092-939-1ISSN1609-042X, 2006.

Evans, W. J. F. and Llewellyn, E. J.: Molecular oxygen emissions in the airglow, Ann. Géophys., Tome 26, 167-178, 1970.

Festou, M. C., Atreya, S. K., Donahue, T. M., Sandel, B. R., Shemansky, D. E., and Broadfoot, A. L.: Composition and thermal profiles of the Jovian upper atmosphere determined by the Voyager ultraviolet stellar occultation experiment, J. Geophys. Res., 86, 5715-5725, 1981.

Fioletov, V. E. and Shepherd, T. G.: Summertime total ozone variations over middle and polar latitudes, Geophys. Res. Lett., 32, L04807, doi:10.1029/2004GL022080, 2005.

Fischer, H., Birk, M., Blom, C., Carli, B., Carlotti, M., von Clarmann, T., Delbouille, L., Dudhia, A., Ehhalt, D., Endemann, M., Flaud, J. M., Gessner, R., Kleinert, A., Koopman, R., Langen, J., López-Puertas, M., Mosner, P., Nett, H., Oelhaf, H., Perron, G., Remedios, J., Ridolfi, M., Stiller, G., and Zander, R.: MIPAS: an instrument for atmospheric and climate research, Atmos. Chem. Phys., 8, 2151-2188, doi:10.5194/acp-8-21512008, 2008.

Fortuin, J. P. F. and Kelder, H.: An ozone climatology based on ozonesonde and satellite measurements, J. Geophys. Res., 103(D24), 31709-31734, doi:10331709-31734, 1998.

Frederick, J. E.: Measurement Requirements for the detection of ozone trends, ozone Correlative Measurements Workshop, NASA Conference publication 2362, Appendix 3, 1984.

Fritts, D. C. and Alexander, M. J.: Gravity wave dynamics and effects in the middle atmosphere, Rev. Geophys., 41(1), 1003, doi:10.1029/2001RG000106, 2003.
Fussen, D., Vanhellemont, F., and Bingen, C.: Evolution of stratospheric aerosols in the post-Pinatubo period measured by solar occultation, Atmos. Environ., 35, 5067-5078, 2001.

Fussen, D., Vanhellemont, F., Bingen, C., Kyrölä, E., Tamminen, J., Sofieva, V., Hassinen, S., Seppala, A., Bertaux, J. L., Hauchecorne, A., Dalaudier, F., Renard, J. B., Fraisse, R., Fanton d'Andon, O., Barrot, G., A. Mangin, Theodore, B., Guirlet, M., Snoeij, P., Koopman R., and Saavedra, L., Global measurement of the mesospheric sodium layer by the star occultation instrument GOMOS, Geophys. Res. Lett., 31, L24110, doi:10.1029/2004GL021618, 2004.

Fussen, D., Vanhellemont, F., Dodion, J., Bingen, C., Walker, K. A., Boone, C. D., McLeod, S. D., and Bernath, P. F.: Intercomparison of ozone and nitrogen dioxide concentration profiles retrieved by the ACE and GOMOS occultation experiments, Geophys. Res. Lett., 32, L16S02, doi:10.1029/2005GL022468, 2005.

Fussen, D., Vanhellemont, F., Dodion, J., Bingen, C., Mateshvili, N., Daerden, F., Fonteyn, D., Errera, Q., Chabrillat, S., Kyrölä, E., Tamminen, J., Sofieva, V., Hauchecorne, A., Dalaudier, F., Bertaux, J.-L., Renard, J.-B., Fraisse, R., d'Andon, O. F., Barrot, G., Guirlet, M., Mangin, A., Fehr, T., Snoeij, P.,and Saavedra, L.: A global OClO stratospheric layer discovered in GOMOS stellar occultation measurements, Geophys. Res. Lett., 33, L13815, doi:10.1029/2006GL026406, 2006.

Fussen, D., Vanhellemont, F., Tétard, C., Mateshvili, N., Dekemper, E., Loodts, N., Bingen, C., Kyrölä, E., Tamminen, J., Sofieva, V., Hauchecorne, A., Dalaudier, F., Bertaux, J.-L., Barrot, G., Blanot, L., Fanton d'Andon, O., Fehr, T., Saavedra, L., Yuan, T., and She, C.-Y.: A global climatology of the mesospheric sodium layer from GOMOS data during the 2002-2008 period, Atmos. Chem. Phys. Discuss., 10, 6097-6127, doi:10.5194/acpd-106097-2010, 2010.

Garcia, R. R. and Solomon, S.: The effect of breaking gravity waves on the dynamics and chemical composition of the mesosphere and lower thermosphere. J. Geophys Res., 90, 3850-3868, 1985.

Gumbel, J., Fan, Z. Y., Waldemarsson, T., Stegman, J., Witt, G., Llewellyn, E. J., She, C. Y., and Plane, J. M. C.: Retrieval of global mesospheric sodium densities from the Odin satellite, Geophys. Res. Lett. 34, L04813, doi:10.1029/2006GL028687, 2007.

Gurvich, A. S. and Kan, V.: Structure of air density irregularities in the stratosphere from spacecraft observations of stellar scintillation, 1. Three-dimensional spectrum model and recovery of its parameters, Izv. Russ. Acad. Sci. Atmos. Oceanic Phys., 39, 300-310, 2003a.

Gurvich, A. S. and Kan, V.: Structure of air density irregularities in the stratosphere from spacecraft observations of stellar scintillation, 2. Characteristic scales, structure characteristics, and kinetic energy dissipation, Izv. Russ. Acad. Sci. Atmos. Oceanic Phys., 39, 311-321, 2003b.

Gurvich, A. S., Kan, V., Savchenko, S. A., Pakhomov, A. I., and Padalka, G. I.: Studying the turbulence and internal waves in the stratosphere from spacecraft observations of stellar scintillation: I. Experimental technique and analysis of the scintillation variance, Izv. Russ. Acad. Sci. Atmos. Oceanic Phys., Engl. Transl., 37, 436-451, 2001a.

Gurvich, A. S., Kan, V., Savchenko, S. A., Pakhomov, A. I., and Padalka, G. I.: Studying the turbulence and internal waves in the 
stratosphere from spacecraft observations of stellar scintillation: II. Probability distributions and scintillation spectra, Izv. Russ. Acad. Sci. Atmos. Oceanic Phys., Engl. Transl., 37, 452-465, $2001 b$.

Gurvich, A. S., Dalaudier, F., and Sofieva, V. F.: Study of stratospheric air density irregularities based on two wavelength observation of stellar scintillation by Global Ozone Monitoring by Occultation of Stars (GOMOS) on Envisat, J. Geophys. Res., 110, D11110, doi:10.1029/2004JD005536, 2005.

Gurvich, A. S., Sofieva, V. F., and Dalaudier, F.: Global distribution of $C_{T}^{2}$ at altitudes $30-50 \mathrm{~km}$ from space-borne observations of stellar scintillation, Geophys. Res. Lett., 34, L24813, doi:10.1029/2007GL031134, 2007.

Hartogh, P., Jarchow, C., Sonnemann, G. R., and Grygalashvyly, M.: On the spatiotemporal behavior of ozone within the upper mesosphere/mesopause region under nearly polar night conditions, J. Geophys. Res., 109, D18303, doi:10.1029/2004JD004576, 2004.

Hauchecorne, A., Bertaux, J., Dalaudier, F., Cot, C., Lebrun, J., Bekki, S., Marchand, M., Kyrola, E., Tamminen, J., Sofieva, V., Fussen, D., Vanhellemont, F., d'Andon, O., Barrot, G., Mangin, A., Theodore, B., Guirlet, M., Snoeij, P., Koopman, R., de Miguel, L., Fraisse, R., and Renard, J.: First simultaneous global measurements of nighttime stratospheric $\mathrm{NO}_{2}$ and $\mathrm{NO}_{3}$ observed by Global Ozone Monitoring by Occultation of Stars (GOMOS)/Envisat in 2003, J. Geophys. Res.-Atmos. 110, D18301, doi:10.1029/2004JD005711, 2005.

Hauchecorne, A., Lallement, R., and Bertaux, J. L.: Impact of solar activity on stratospheric ozone and $\mathrm{NO}_{2}$ observed by GOMOS, Space Sci. Rev., 125, 393-402, 2006.

Hauchecorne, A., Bertaux, J. L., Dalaudier, F., Russell III, J. M., Mlynczak, M. G., Kyrölä, E., and Fussen, D.: Large increase of $\mathrm{NO}_{2}$ in the north polar mesosphere in January-February 2004: Evidence of a dynamical origin from GOMOS/ENVISAT and SABER/TIMED data, Geophys. Res. Lett., 34, L03810, doi:10.1029/2006GL027628, 2007.

Hauchecorne, A., Bertaux, J. L., Dalaudier, F., Keckhut, P., Lemennais, P., Bekki, S., Marchand, M., Lebrun, J. C., Kyrölä, E., Tamminen, J., Sofieva, V., Fussen, D., Vanhellemont, F., Fanton d'Andon, O., Barrot, G., Blanot, L., Fehr, T., and Saavedra de Miguel, L.: Response of tropical stratospheric $\mathrm{O}_{3}$, $\mathrm{NO}_{2}$ and $\mathrm{NO}_{3}$ to the equatorial Quasi-Biennial Oscillation and to temperature as seen from GOMOS/ENVISAT, Atmos. Chem. Phys., 10, 8873-8879, doi:10.5194/acp-10-8873-2010, 2010.

Hays, P. B. and Roble, R. G.: Stellar spectra and Atmospheric Composition, J. Atmos. Sci., 25, p. 141, 1968.

Hays, P. B. and Roble, R. G.: Observation of mesospheric ozone at low latitudes, Planet. Space Sci., 21, 273-279, 1973.

Hedin, A. E.: Extension of the MSIS thermosphere model into the middle and lower atmosphere, J. Geophys. Res., 96, 1159-1162, 1991.

Holton, J. R.: An Introduction to Dynamic Meteorology, Int. Geophys., 88, 4th edn., Elsevier, New York, USA, 2004.

Jiang, J. H., Wang, B., Goya, K., Hocke, K., Eckermann, S. D., Ma, J., Wu, D. L., and Read, W. G.: Geographical distribution and interseasonal variability of tropical deep convection: UARS MLS observations and analyses, J. Geophys. Res., 109, D03111, doi:10.1029/2003JD003756, 2004.
Kan, V., Dalaudier, F., and Gurvich, A. S.: Chromatic refraction with Global Ozone Monitoring by Occultation of Stars. II. Statistical properties of scintillations, Appl. Optics, 40(6), 87889, 2001.

Keckhut, P., Hauchecorne, A., Blanot, L., Hocke, K., GodinBeekmann, S., Bertaux, J.-L., Barrot, G., Kyrölä, E., van Gijsel, A., and Pazmino, A.: Ozone monitoring with the GOMOSENVISAT experiment version 5, Atmos. Chem. Phys. Discuss., 10, 14713-14735, doi:10.5194/acpd-10-14713-2010, 2010.

Korablev, O., Bertaux, J. L., Dubois, J. P.: Occultation of stars in the UV: Study of the atmosphere of Mars, J. Geophys. Res. Planets, 106, E4, 7597-7610, 2001.

Kyrölä, E., Tamminen, J., Leppelmeier, G. W., Sofieva, V., Hassinen, S., Bertaux, J. L., Hauchecorne, A., Dalaudier, F., Cot, C., Korablev, O., d'Andon, O. F., Barrot, G., Mangin, A., Theodore, B., Guirlet, M., Etanchaud, F., Snoeij, P., Koopman, R., Saavedra, L., Fraisse, R., Fussen, D., and Vanhellemont, F.: GOMOS on Envisat: An overview, Adv. Space Res., 33, 10201028, 2004.

Kyrölä, E., Tamminen, J., Leppelmeier, G. W., Sofieva, V., Hassinen, S., Seppälä, A., Verronen, P. T., Bertaux, J.L., Hauchecorne, A., Dalaudier, F., Fussen, D., Vanhellemont, F., Fanton d'Andon, O., Barrot, G., Mangin, A., Theodore, B., Guirlet, M., Koopman, R., Saavedra, L., Snoeij, P., and Fehr, T.: Nighttime ozone profiles in the stratosphere and mesosphere by the Global Ozone Monitoring by Occultation of Stars on Envisat, J. Geophys. Res., 111, D24(306), doi:10.1029/2006JD007193, 2006.

Kyrölä, E., Tamminen, J., Sofieva, V., Bertaux, J. L., Hauchecorne, A., Dalaudier, F., Fussen, D., Vanhellemont, F., Fanton d'Andon, O., Barrot, G., Guirlet, M., Mangin, A., Blanot, L., Fehr, T., Saavedra de Miguel, L., and Fraisse, R.: Retrieval of atmospheric parameters from GOMOS data, Atmos. Chem. Phys. Discuss., 10, 10145-10217, doi:10.5194/acpd-10-10145-2010, 2010a.

Kyrölä, E., Tamminen, J., Sofieva, V., Bertaux, J. L., Hauchecorne, A., Dalaudier, F., Fussen, D., Vanhellemont, F., Fanton d'Andon, O., Barrot, G., Guirlet, M., Fehr, T., and Saavedra de Miguel, L.: GOMOS $\mathrm{O}_{2}, \mathrm{NO}_{2}$, and $\mathrm{NO}_{3}$ observations in 2002-2008, Atmos. Chem. Phys. Discuss., 10, 2169-2220, doi:10.5194/acpd-102169-2010, 2010b.

Marchand, M., Bekki, S., Hauchecorne, A., and Bertaux J. L.: Validation of the self-consistency of GOMOS $\mathrm{NO}_{3}, \mathrm{NO}_{2}$ and $\mathrm{O}_{3}$ data using chemical data assimilation, Geophys. Res. Lett., 31, L10107, doi:10.1029/2004GL019631, 2004.

Marchand, M., Bekki, S., Lefèvre, F., and Hauchecorne, A. Temperature retrieval from stratospheric $\mathrm{O} 3$ and NO3 GOMOS data, Geophys. Res. Lett., 34, L24809, doi:10.1029/2007GL030280, 2007.

Mégie, G. and Blamont, J. E.: Laser sounding of atmospheric sodium: Interpretation in terms of global atmospheric parameters, Planet. Space Sci., 25, 1093-1109, 1977.

Meijer, Y. J., Swart, D. P. J., Allaart, M., Andersen, S.B., Bodeker, G., Boyd, G., Braathena, Calisesia, Y., Claude, H., Dorokhov, V. von der Gathen, P., Gil, M., Godin-Beekmann, S., Goutail, F. , Hansen, G., Karpetchko, A., Keckhut, P., Kelder, H. M., Koelemeijer, R., Kois, B., Koopman, R.M., Lambert, J.-C., Leblanc, T., McDermid, I. S., Pal, S., Kopp, G., Schets, H., Stubi, R., Suortti, T., Visconti, G., and Yela, M.: Pole-to-pole validation of ENVISAT/GOMOS ozone profiles using data from 
ground-based and balloon-sonde measurements, J.Geophys. Res., 109(D23), D23305, doi:10.1029/2004JD004834, 2004.

Montoux, N., Hauchecorne, A., Pommereau, J.-P., Lefèvre, F., Durry, G., Jones, R. L., Rozanov, A., Dhomse, S., Burrows, J. P., Morel, B., and Bencherif, H.: Evaluation of balloon and satellite water vapour measurements in the Southern tropical and subtropical UTLS during the HIBISCUS campaign, Atmos. Chem. Phys., 9, 5299-5319, doi:10.5194/acp-9-5299-2009, 2009.

Naudet, J. P., Rigaud, P., and Pirre, M.: Altitude Distribution of Stratospheric $\mathrm{NO}_{3}$. 1. Observations of $\mathrm{NO}_{3}$ and related species, J. Geophys. Res., 94, p. 6374, 1989.

Pérot, K., Hauchecorne, A., Montmessin, F., Bertaux, J.-L., Blanot, L., Dalaudier, F., Fussen, D., and Kyrölä, E.: First climatology of polar mesospheric clouds from GOMOS/ENVISAT stellar occultation instrument, Atmos. Chem. Phys., 10, 2723-2735, doi:10.5194/acp-10-2723-2010, 2010.

Plane, J. M. C.: A time-resolved model of the mesospheric Na layer: constraints on the meteor input function, Atmos. Chem. Phys., 4 , 627-638, doi:10.5194/acp-4-627-2004, 2004.

Pommereau, J. P. and Piquard J.: Observations of the vertical distribution of stratospheric OClO, Geophys. Res. Lett., 21, 1231-1234, 1994.

Reburn, W. J., Remedios, J. J., Morris, P. E., Rodgers, C. D., Taylor, F. W., Kerridge, B. J., Knight, R. J., Ballard, J., Kumer, J. B., and Massie, S. T.: Validation of $\mathrm{NO}_{2}$ measurements from the ISAMS, J. Geophys. Res., 101, 9873-9895, 1996.

Renard, J.-B., Lefèvre, F., Pirre, M., Robert, C., and Huguenin, D.: Vertical profile of night-time stratospheric OClO, J. Atmos. Chem., 26, 65-76, 1997a.

Renard, J. B., Pirre, M., Robert, C., Lefevre, F., Lateltin, E., Nozière, B., and Huguenin, D.: Vertical distribution of night time stratospheric $\mathrm{NO}_{2}$ from balloon measurements: comparison with models, Geophys. Res. Lett., 24, p. 73, 1997b.

Renard, J.-B., Chipperfield, M., Berthet, G., Goffinont-Taupin, F., Robert, C., Chartier, M., Roscoe, H., Feng, W., Rivière, E., and Pirre, $\mathrm{M} .: \mathrm{NO}_{3}$ vertical profile measurements from remote sensing balloon-borne spectrometers and comparison with model calculations, J. Atmos. Chem., 51(1), 65-78, 2005.

Renard, J. B., Berthet, G., Brogniez, C., Catoire, V., Fussen, D., Goutail, F., Oelhaf, H., Pommereau,J. P., Roscoe, H. K., Wetzel, G., and 8 coauthors: Validation of GOMOS-Envisat vertical profiles of $\mathrm{O}_{3}, \mathrm{NO}_{2}, \mathrm{NO}_{3}$, and aerosol extinction using balloon-borne instruments and analysis of the retrievals, J. Geophys. Res., 113(A2), doi:10.1029/2007JA012345, 2008.

Riegler, G. R., Liu, S. C., Cicerone, R. J., and Drake, J. F.: Stellar Occultations Measurements of Atmospheric Ozone and Chlorine from OAO 3, J. Geophys. Res., 81, p. 4997, doi:10.1029/JA081i028p04997, 1976.

Riegler, G. R., Liu, S. C., Wasser, B., Atreya, S. K., Donahue, T. M., and Drake, J. F.: UV stellar occultation measurements of night time equatorial ozone, Geophys. Res. Lett., 4, 145-162 1977.

Roscoe, H. K., Taylor, W. H., Evans, J. D., Tait, A. M., Freshwater, R., Fish, D., Strong, E. K., and. Jones, R. L.: Automated groundbased star-pointing UV-visible spectrometer for stratospheric measurements, Appl. Optics, 36, 6069-6075, 1997.
Russell III, J. M., Bailey, S. M., Gordley, L. L., Rusch, D. W., Horanyi, M., Hervig, M. E., Thomas, G. E., Randall C. E., Siskind, D. E., Stevens, M. H., Summers, M. E., Taylor, M. J., Englert, C. R., Espy, P. J., McClintock, W. E., and Merkel, A. W.: The Aeronomy of Ice in the Mesosphere (AIM) mission: overview and early science results, J. Atmos. Terr. Phys., 71(34), 289-299, 2009.

Seppälä, A., Verronen, P. T., Kyrölä, E., Hassinen, S., Backman, L., Hauchecorne, A., Bertaux, J. L., and Fussen, D.: Ozone depletion in the Northern Hemisphere polar winter as seen by GOMOS/Envisat, Geophys. Res.Lett., 31, L19107, doi:10.1029/2004GL021042, 2004.

Seppälä, A., Verronen, P. T., Sofieva, V. F., Tamminen, J., Kyrola, E., Rodger, C. J., and Clilverd, M. A.: Destruction of the Tertiary Ozone Maximum During a Solar Proton Event, Geophys. Res. Lett., 33, L07804, doi:10.1029/2005GL025571, 2006.

Sessler, J., Chipperfield, M. P., Pyle, J. A., and Toumi, R.: Stratospheric $\mathrm{OClO}$ measurements as a poor quantitative indicator of chlorine activation, Geophys. Res. Lett., 22, 687690, 1995.

She, C. Y., Chen, S. S., Hu, Z. L., Sherman, J., Vance, J. D., Vasoli, V., White, M. A., Yu, J. R., and Krueger, D. A.: Eight-year climatology of nocturnal temperature and sodium density in the mesopause region (80 to $105 \mathrm{~km})$ over Fort Collins, $\mathrm{CO}\left(41^{\circ} \mathrm{N}\right.$, $105^{\circ}$ W), Geophys. Res. Lett., 27, 3289-3292, 2000.

Smith, A. K.: Physics and chemistry of the mesopause region, J. Atmos. Sol.-Terr. Phy., 66, 839-857, 2004.

Smith, G. R. and Hunten, D. M.: Study of Planetary Atmospheres by Absorptive Occultations, Rev. Geophys., 28, p. 117, 1990.

Shemansky, D. E., Stewart, A. I. F., West, R. A., Esposito, L. W., Hallett, J. T., and Xianming, L.: The Cassini UVIS stellar probe of the Titan atmosphere, Science, 308(5724), 978-982, 2005.

Sofieva, V. F., Verronen, P. T., Kyrölä, E., Hassinen, S., and GOMOS CAL/VAL team: The tertiary ozone maximum in the middle mesosphere as seen by GOMOS on Envisat; Quadrennial Ozone Symposium, 2004b.

Sofieva, V. F., Kyrölä, E., Hassinen, S., Backman, L., Tamminen, J., Seppälä, A. , Thölix, L., Gurvich, A. S., Kan, V., Dalaudier, F., Hauchecorne, A., Bertaux, J.-L., Fussen, D., Vanhellemont, F., Fanton d'Andon, O., Barrot, G., Mangin, A., Guirlet, M., Fehr, T., Snoeij, P., Saavedra, L., Koopman, R., and Fraisse, R.: Global analysis of scintillation variance: Indication of gravity wave breaking in the polar winter upper stratosphere, Geophys. Res. Lett., 34, L03812, doi:10.1029/2006GL028132, 2007 a.

Sofieva, V. F., Gurvich, A. S., Dalaudier, F., and Kan, V.: Reconstruction of internal gravity wave and turbulence parameters in the stratosphere using GOMOS scintillation measurements, J. Geophys. Res., 112, D12113, doi:10.1029/2006JD007483, 2007b.

Sofieva, V. F., Dalaudier, F., Kivi, R., and Kyrö, E.: On the variability of temperature profiles in the stratosphere: Implications for validation, Geophys. Res. Lett., 35, L23808, doi:10.1029/2008GL035539, 2008.

Sofieva, V. F., Gurvich, A. S., and Dalaudier, F.: Gravity wave spectra parameters in 2003 retrieved from stellar scintillation measurements by GOMOS, Geophys. Res. Lett., 36, L05811, doi:10.1029/2008GL036726, 2009a. 
Sofieva, V. F., Kyrölä, E., Verronen, P. T., Seppälä, A., Tamminen, J., Marsh, D. R., Smith, A. K., Bertaux, J.-L., Hauchecorne, A., Dalaudier, F., Fussen, D., Vanhellemont, F., Fanton d'Andon, O., Barrot, G., Guirlet, M., Fehr, T., and Saavedra, L.: Spatio-temporal observations of the tertiary ozone maximum, Atmos. Chem. Phys., 9, 4439-4445, doi:10.5194/acp-9-44392009, 2009b.

Sofieva, V. F., Kan, V., Dalaudier, F., Kyrölä, E., Tamminen, J., Bertaux, J.-L., Hauchecorne, A., Fussen, D., and Vanhellemont, F.: Influence of scintillation on GOMOS ozone retrievals, Atmos. Chem. Phys. Discuss., 9, 12615-12643, doi:10.5194/acpd-912615-2009, 2009c.

Sofieva, V. F., Vira, J., Dalaudier, F., Hauchecorne A., and the GOMOS team: Validation of GOMOS/Envisat high-resolution temperature profiles (HRTP) using spectral analysis, in New Horizons in Occultation Research, Studies in Atmosphere and Climate, edited by: Steiner, A., Pirscher, B., Foelsche, U., and Kirchengast, G., Springer-Verlag, Berlin, Heidelberg, Germany, 97-107, doi:10.1007/978-3-642-00321-9_9, 2009d.

Tamminen, J., Kyrölä, E., Sofieva, V. F., Laine, M., Bertaux, J.L., Hauchecorne, A., Dalaudier, F., Fussen, D., Vanhellemont, F., Fanton-d'Andon, O., Barrot, G., Mangin, A., Guirlet, M., Blanot, L., Fehr, T., Saavedra de Miguel, L., and Fraisse, R.: GOMOS data characterization and error estimation, Atmos. Chem. Phys. Discuss., 10, 6755-6796, doi:10.5194/acpd-106755-2010, 2010.

Tétard, C., Fussen, D., Bingen, C., Capouillez, N., Dekemper, E., Loodts, N., Mateshvili, N., Vanhellemont, F., Kyrölä, E., Tamminen, J., Sofieva, V., Hauchecorne, A., Dalaudier, F., Bertaux, J.-L., Fanton d'Andon, O., Barrot, G., Guirlet, M., Fehr, T., and Saavedra, L.: Simultaneous measurements of $\mathrm{OClO}, \mathrm{NO}_{2}$ and $\mathrm{O}_{3}$ in the Arctic polar vortex by the GOMOS instrument, Atmos. Chem. Phys., 9, 7857-7866, doi:10.5194/acp-9-78572009, 2009.

Thomas, G. E.: Mesospheric Clouds and the Physics of the Mesopause Region, Rev. Geophys., 29, 553-575, 1991.

Thomas, G. E. and Olivero, J. J.: Noctilucent clouds as possible indicators of global change in the mesosphere, Adv. Space Res., 28(7), 937-946, 2001.

Thomason, L. W. and Taha, G.: SAGE III aerosol extinction measurements: Initial results, Geophys. Res. Lett., 30, 1631, doi:10.1029/2003GL017317, 2003.

Tie, X., Brasseur, G., Hess, P., and Riese, M.: Hemispheric asymmetry of chemical species and its effect on stratospheric ozone: emphasis on halogen loading, Adv. Space Res., 24, 16311636, 1999.

Tukiainen, S., Hassinen, S., Seppälä, A., Auvinen, H., Kyrölä, E., Tamminen, J., Haley, C. S., Lloyd, N., and Verronen, P. T.: Description and validation of a limb scatter retrieval method for Odin/OSIRIS, J. Geophys. Res. Atmos., 113, D04308, 12 pp., doi:10.1029/2007JD008591, 2008.
Vanhellemont, F., Fussen, D., Bingen, C., Kyrölä, E., Tamminen, J., Sofieva, V., Hassinen, S., Verronen, P., Seppälä, A., Bertaux, J. L., Hauchecorne, A., Dalaudier, F., Fanton d'Andon, O., Barrot, G., Mangin, A., Theodore, B., Guirlet, M., Renard, J. B., Fraisse, R., Snoeij, P., Koopman, R., and Saavedra, L.: A 2003 stratospheric aerosol extinction and PSC climatology from GOMOS measurements on Envisat, Atmos. Chem. Phys., 5, 2413-2417, doi:10.5194/acp-5-2413-2005, 2005.

Vanhellemont, F., Fussen, D., Mateshvili, N., Tétard, C., Bingen, C., Dekemper, E., Loodts, N., Kyrölä, E., Sofieva, V., Tamminen, J., Hauchecorne, A., Bertaux, J.-L., Dalaudier, F., Blanot, L., Fanton d'Andon, O., Barrot, G., Guirlet, M., Fehr, T., and Saavedra, L.: Optical extinction by upper tropospheric/stratospheric aerosols and clouds: GOMOS observations for the period 2002-2008, Atmos. Chem. Phys., 10, 7997-8009, doi:10.5194/acp-10-7997-2010, 2010.

Verronen, P. T., Kyrölä, E., Tamminen, J., Funke, B., Gil-Lopez, S., Kaufmann, M., Lopez-Puertas, M., von Clarmann, T., Stiller, G., Grabowski, U. and Höpfner, M.: A comparison of nighttime GOMOS and MIPAS ozone profiles in the stratosphere and mesosphere, Adv. Space Res., 36, 958-966, 2005 a.

Verronen, P. T., Seppälä, A., Clilverd, M. A., Rodger, C. J., Kyrölä, E., Enell, C.-F., Ulich, Th., and Turunen, E.: Diurnal variation of ozone depletion during the October-November 2003 solar proton events, J. Geophys. Res., 110, A09S32, doi:10.1029/2004JA010932, 2005b.

Verronen, P. T., Seppälä, A., Kyrölä, E., Tamminen, J., Pickett, H. M., and Turunen, E.: Production of Odd Hydrogen in the Mesosphere During the January 2005 Solar Proton Event, Geophys. Res. Lett., 33, L24811, doi:10.1029/2006GL028115, 2006.

Verronen, P. T., Ceccherini, S., Cortesi, U., Kyrölä, E., and Tamminen, J.: Statistical comparison of night-time $\mathrm{NO}_{2}$ observations in 2003-2006 from GOMOS and MIPAS instruments, Adv. Space Res., 43, 1918-1925, doi:10.1016/j.asr.2009.01.027, 2009.

Vervack, R. J., Yee, J. H., Carbary, J. F., and Morgan, F.: Atmospheric remote sensing using a combined extinctive and refractive stellar occultation technique 3 . Inversion method for refraction measurements, J. Geophys. Res. Atmos., 107(D15), 4261, doi:10.1029/2001JD000796, 2002.

WMO (World Meteorological Organization): Scientific Assessment of Ozone Depletion: 2006, Global Ozone Research and Monitoring Project - Report No. 50, 572 pp., Geneva, 2007

Yee, J. H., Vervack, R. J. DeMajistre, R. Morgan, F., Carbary, J. F., Romick, G. J., Morrison, D., Lloyd, S. A., DeCola, P. L., Paxton, L. J., Anderson, D. E., Kumar, C. K., and Meng, C.I.: Atmospheric remote sensing using a combined extinctive and refractive stellar occultation technique 1 . Overview and proof-ofconcept observations, J. Geophys. Res. Atmos., 107(D14), 4213, doi:10.1029/2001JD000794, 2002. 